\title{
The dynamics of 1D Bloch electrons in constant electric fields
}

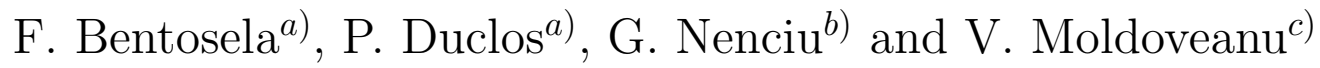

October 8, 2018

\begin{abstract}
${ }^{a)}$ Centre de Physique Théorique UMR 6207 - Unité Mixte de Recherche du CNRS et des Universités Aix-Marseille I, Aix-Marseille II et de l'Université du Sud Toulon-Var - Laboratoire affilié à la FRUMAM, F-13288 Marseille Cedex 9, France

b) Department of Theoretical Physics, University of Bucharest, P.O. MG 11, 76900 - Bucharest, Romania and Institute of Mathematics of the Romanian Academy, P.O. Box 1-764, RO70700 Bucharest, Romania.

${ }^{c)}$ Group of Theoretical Physics, National Institute of Materials Physics, P.O.Box $M G-7$, Bucharest, Romania.
\end{abstract}

\begin{abstract}
We study the dynamics of a 1D Bloch electron subjected to a constant electric field. The periodic potential is supposed to be less singular than the $\delta$-like potential (Dirac comb). We give a rigorous proof of Ao's result 2] that for a large class of initial conditions (high momentum regime) there is no localization in momentum space. The proof is based on the mathematical substantiation of the two simplifying assumptions made in physical literature: the transitions between far away bands can be neglected and the transitions at the quasi-crossing can be described by Landau-Zener like formulae. Using the connection between the above model and the driven quantum ring (DQR) shown by Avron and Nemirovski [5], our results imply the increase of energy for weakly singular such DQR and appropiate initial conditions.
\end{abstract}

\section{Introduction}

The dynamics of Bloch electrons (i.e electrons subjected to a periodic potential) in the presence of a homogeneous electric field is among those topics that appeared since the beginning of the quantum theory of the solid state physics but are still alive today for both mathematical and physical reasons; in particular the emergence of superlattices leads to interesting physics (see e.g [30]) 
The dynamics of an electron in one dimension subjected to a periodic potential $V_{\text {per }}(x)$ and a constant electric field $E=-e F$ is described by the time-dependent Schrödinger equation:

$$
i \hbar \frac{\partial \psi}{\partial t}(x, t)=H^{S W} \psi(x, t)=\left(-\frac{\hbar^{2}}{2 m} \frac{d^{2}}{d x^{2}}-e F x+V_{\text {per }}(x)\right) \psi(x, t)
$$

where $H^{S W}$ is the so-called Stark-Wannier Hamiltonian.

There is a large body of mathematically oriented literature about spectral properties of the Stark-Wannier Hamiltonian (especially concerning the Stark-Wannier ladder problem; see [8, 9, 10, 22] and references therein). In particular the spectrum has been proved to be absolutely continuous for twice differentiable potentials [7] and recently, results about the nature of the spectrum were obtained for more singular potentials [17, 16, 26, 4.

On the contrary, at the rigorous level there are by far fewer results concerning the dynamics generated by $H^{S W}$. Of course, as well known, the results about spectral properties of $H^{S W}$ lead to results about dynamics but they either concern finite (albeit long) intervals of time [22, 23] or are only qualitative. As an example we mention the results of Avron and Nemirovski [5] on driven quantum rings. They first proved the nice result that there is a close connection between driven quantum rings and Stark-Wannier Hamiltonians. Then, using this connection and the fact mentioned above that the spectrum of $H^{S W}$ is absolutely continuous for twice differentiable potentials they proved that the energy of a smoothly driven quantum ring grows indefinitely as $t \rightarrow \infty$ for arbitrary initial conditions.

At the physical level the dynamics generated by $H^{S W}$ has been thoroughly studied (using the temporal gauge representation) both by analytical and numerical methods. Due to the difficulty of the problem two main assumptions are made:

1. The only interband transitions considered are the ones between neighbouring bands ( the so-called Zener tunneling process through avoided crossings); all the others are considered sufficiently small to be neglected.

2. The transition probabilities can be computed approximatively via LandauZener type formulae.

Based on earlier developements [12, the consequences of these assumptions were exhaustively discussed by Ao [2]. More precisely, writing the evolution at arbitrary times as a product of evolutions over half Bloch periods and using for the last ones the "scattering matrix" between adjacent bands given by Blatter and Browne [12] he reduced the original problem to a discrete dynamics, amenable to numerical and analytical study. The main result coming from his analysis is that for potentials more regular than the Dirac comb and a large class of initial conditions there is a propagating front for the Bloch electron (in other words it will escape at infinity). The Dirac comb is a critical border: one could have either propagation or localization (respectively pure point, continuous spectrum spectrum or even mixt cases ) depending upon the electric field strength and some resonance conditions.

At the rigorous level the existence of a propagating front (with quantitative estimates) for a large class of initial conditions as well as some spectral consequences 
has been recently proved in [3] for potentials more regular than the $L_{\text {loc }}^{2}$ class.

The aim of this paper is to provide a rigorous justification of the simplifying assumptions 1 and 2 above in order to substantiate from the mathematical point of view at least a part of Ao's analysis. We do it for a class of periodic potentials whose Fourier coefficients, $\hat{V}(n)$, satisfy $|\hat{V}(n)|<$ const. $|n|^{-r}$ for all $r>0$. Notice however that the results of sections 3 and 4 are even valid for $r>-\frac{1}{2}$.

The need for a rigorous control comes from the fact that even if the errors involved in the assumptions 1 and 2 are "small" over a half Bloch period they acummulate during a long time evolution to the point of making irrelevant the approximate computation based on the discrete dynamics.

The problem turned out to be fairly complex for two reasons. First we are dealing with singular periodic potentials which leads to a definition in the form sense of the gauged Hamiltonian $\tilde{H}^{S W}$ and secondly, we want to control the evolution over infinite intervals of time which demands a very good control of the errors involved.

The results in this paper allows us to prove the existence of a propagating front (which in turn implies the existence of continuous spectrum for $H^{S W}$ ) up to the Dirac comb (which corresponds to $\hat{V}(n)=1$ and is known as notoriously difficult). Actually the Dirac comb case remains open, although it is not clear for us whether the present approach could provide as well some results in this case.

The plan of the paper is as follows. Section 2 contains the preliminaries: the direct integral representation of the Stark-Wannier Hamiltonian in the temporal gauge, the periodicity in time properties of the fiber Hamiltonian and the reduction to the one Bloch period. All that is done for $\tilde{H}^{S W}$ defined as a quadratic form sum. Section 2 contains in addition a key technical estimate (Lemma 2.3) used many times in the following sections. Since the proof is technical and somehow long it was moved to Appendix 7.1.

Section 3 contains the first main result of our paper (Theorem 3.1) saying that the transitions over a half Bloch period, between far-away bands (neglected by assumption 1 in the physical literature ) can be controlled by adiabatic techniques. The idea is that in adiabatic perturbation theory the relevant parameter is $\varepsilon / \Delta$, where $\varepsilon$ is the slowness parameter and $\Delta$ is the spectral gap. In our case it is the gap that grows with energy making thus the adiabatic machinery effective.

After the far-away decoupling in Section 3 we come to the "in-band" dynamics i.e to Zener transitions through quasi-crossings at high energies. The task of computing this dynamics has been taken again and again in the physical literature. It consists in solving a $2 \times 2$ system of first order ordinary differential equations over a finite interval of time. There are two problems here. The first is that the coefficients are taken as given by a low order almost degenerate pertubation theory without any control of the reminder. Secondly, the obtained "Zener model" is solved over an infinite period of time. We cope with the first problem by using the reduction theory in Section 4 to obtain an effective Hamiltonian (Theorem 4.1). Then we integrate the system over one half period by the use of Dyson series; we compute the first two terms in Section 5 and estimate the remainder in Appendix 7.3. The resulting "transfer matrix" is given in Theorem 5.1. We expect the results mentioned 
above to give a lot of information on spectral and dynamical properties of the StarkWannier (SW) and driven quantum ring models for fairly singular potentials. As an example in Section 6 the results in the previous sections are assembled to prove the Ao's statement about the the existence of a propagating front. As direct side consequences we obtain (see Corollary 6.4) that for $r>0$ the continuous spectrum of $H^{S W}$ is not empty (see however the recent paper of Perelman 26] for a better result in this direction), that for a large class of initial conditions the energy of the DQR increases like $t^{2}$ (see Corollary 6.5) and that there is no localization in momentum space (see Corollary 6.6).

In Appendix 7.2 we state for convenience the Sz-Nagÿ transformation matrix. In order to make the reading of the paper easier we start each section by simply stating the theorems and lemmae, their proofs being provided later. 


\section{Preliminaries}

As already said in the introduction we are interested in the evolution given by the Stark-Wannier operator. For simplicity we normalize $e=\hbar=2 m=1$. Moreover since the results we are going to prove along the paper do not depend upon the electric field strength, we set $F=1$. Accordingly the Stark-Wannier Hamiltonian writes

$$
H^{S W}=-\frac{d^{2}}{d x^{2}}-x+V_{\mathrm{per}}
$$

where $V_{\text {per }}$ is a real periodic potential with periodicity $a=2 \pi$. Under these assumptions the quantity $\frac{2 \pi \hbar}{a F}$, the so-called Bloch period, is equal to 1 .

Let us define:

$$
\tilde{U}^{S W}(t, s):=G(t) e^{-i(t-s) H^{S W}} G(s)^{*},
$$

where $G(t)$ is the temporal gauge:

$$
G(t) \psi(x, t):=e^{-i x t} \psi(x, t)=: \tilde{\psi}(x, t) .
$$

Then using (2.2) and (2.3) one has by direct computation:

$$
\begin{aligned}
i \frac{d}{d t} \tilde{U}^{S W}(t, s) & =i\left(\frac{d}{d t} G(t)\right) G(t)^{*} \tilde{U}^{S W}(t, s)+G(t) H^{S W} e^{-i(t-s) H^{S W}} G(s)^{*} \\
& =\left(i\left(\frac{d}{d t} G(t)\right) G(t)^{*}+G(t) H^{S W} G(t)^{*}\right) \tilde{U}^{S W}(t, s) \\
& =\left(\left(-i \frac{d}{d x}+t\right)^{2}+V_{\mathrm{per}}(x)\right) \tilde{U}^{S W}(t, s) .
\end{aligned}
$$

Denoting

$$
\tilde{H}^{S W}(t):=\left(-i \frac{d}{d x}+t\right)^{2}+V_{\mathrm{per}}(x)
$$

we get

$$
i \frac{d}{d t} \tilde{U}^{S W}(t, s)=\tilde{H}^{S W}(t) \tilde{U}^{S W}(t, s)
$$

As well known the periodicity of $V_{\text {per }}$ allows a direct integral representation for $\tilde{H}^{S W}(t)$ via the Fourier-Bloch transform [28] which maps unitarily the space $L^{2}(\mathbb{R})$ onto $L^{2}\left([0,1), d k, l^{2}(\mathbb{Z})\right)$ :

$$
(S \psi)(k, n)=\frac{1}{\sqrt{2 \pi}} \int_{0}^{2 \pi} e^{-i n x}\left\{\sum_{\gamma \in \mathbb{Z}} e^{-i k(x+2 \pi \gamma)} \psi(x+2 \pi \gamma)\right\} d x .
$$

Note that $(S \psi)(k, n)=(\mathcal{F} \psi)(k+n)$ where $\mathcal{F}$ is the Fourier transform. In this representation $\tilde{H}^{S W}(t)$ writes

$$
S \tilde{H}^{S W}(t) S^{*}=\int_{[0,1)}^{\oplus} H(k, t) d k
$$


with the fiber Hamiltonian

$$
H(k, t):=H_{0}(k, t)+V .
$$

We remark that $H(0, t)$ coincide with the Hamiltonian of the DQR when written in the Fourier representation (see e.g [5]) so all the results below on the evolution $U(t, s)$, generated by $H(0, t)$ apply (see the Corollary 6.5 below) to the DQR problem. The unperturbed Hamiltonian $H_{0}(k, t)$ has a simple spectral representation

$$
H_{0}(k, t)=\sum_{n \in \mathbb{Z}} E_{n, 0}(k, t) P_{n, 0}(k)
$$

where

$$
E_{n, 0}(k, t)=(n+k+t)^{2}
$$

and $P_{n, 0}(k):=P_{n, 0}$ is the projection on the $n^{t h}$ vector, $\varphi_{n}$, from the canonical basis in $l^{2}(\mathbb{Z})$

$$
\varphi_{n, 0}(m)=\delta_{n, m}
$$

The perturbation $V$ is given by the following convolution in $l^{2}(\mathbb{Z})$

$$
(V \phi)(k, m)=\sum_{n \in \mathbb{Z}} \hat{V}(m-n) \phi(k, n)
$$

$\hat{V}(n)$ being the Fourier coefficients of $V_{\text {per }}$. Since $V_{\text {per }}$ is real we have $\hat{V}(n)=\overline{\hat{V}}(-n)$. Moreover we can choose $\hat{V}(0)=0$ (this amounts for a shift in the energy scale). We characterize different classes of perturbations by the norm

$$
\|V\|_{r}:=\sup _{n \in \mathbb{Z}}\langle n\rangle^{r}|\hat{V}(n)|<\infty, \quad\langle n\rangle:=\sqrt{1+n^{2}} \quad r \in \mathbb{R} .
$$

Notice that when $r$ gets smaller and smaller, $V_{\text {per }}(x)$ is more and more singular. Actually $V_{\text {per }}(x)$ is to be considered as a tempered distribution on the one dimensional torus $\mathbb{T}$ such that its Fourier series satisfies $\|V\|_{r}<\infty$. For $r>0, V_{\text {per }}(x)$ is realized as an usual function while $r=0$ corresponds to $\delta$-like potentials. For $r>\frac{1}{2}, V_{\text {per }}(x)$ is square integrable over the unit cell, and then as well known [28, $V$ is $H_{0}(k, t)$ bounded with relative bound zero. As a consequence, by the Kato-Rellich theorem [28, $H(k, t)$ is self-adjoint on the (time independent) domain of $H_{0}(k, t)$ :

$$
\mathcal{D}\left(H_{0}(k, t)\right)=\mathcal{H}^{2}=\left\{\phi(n) \mid\langle n\rangle^{2} \phi(n) \in l^{2}(\mathbb{Z})\right\} .
$$

In addition, the existence of a strongly continuous evolution $U(k ; t, s)$, generated by $H(k, t)$ is assured by standard results (see Thm. X.70 in [27]). We are mainly interested in the case of more singular potentials corresponding to $r \leq \frac{1}{2}$ and here the problem is more involved since as one can easily see (take for example $\hat{V}(n)=\langle n\rangle^{-1 / 2}$ ) $V$ is no more $H_{0}(k, t)$-bounded. Notice that the time independent form domain 
of $H_{0}(k, t)$ is $\mathcal{Q}\left(H_{0}(k, t)\right)=\mathcal{H}^{1}=\left\{\phi(n) \mid\langle n\rangle \phi(n) \in l^{2}(\mathbb{Z})\right\}$. Then one can use the theory of Hamiltonians defined as quadratic forms [27]. More precisely, from

$$
R_{0}(z, k, t):=\sum_{n \in \mathbb{Z}} \frac{P_{n, 0}}{E_{n, 0}(k, t)-z}
$$

let us define

$$
\left|R_{0}(z, k, t)\right|^{\frac{1}{2}}=\sum_{n \in \mathbb{Z}} \frac{P_{n, 0}}{\left|E_{n, 0}(k, t)-z\right|^{\frac{1}{2}}}
$$

and

$$
R_{0}(z, k, t)^{\frac{1}{2}}=\sum_{n \in \mathbb{Z}} \frac{\operatorname{sgn}\left(E_{n, 0}(k, t)-z\right) P_{n, 0}}{\left|E_{n, 0}(k, t)-z\right|^{\frac{1}{2}}} .
$$

Then $R_{0}(z, k, t)=R_{0}(z, k, t)^{\frac{1}{2}}\left|R_{0}(z, k, t)\right|^{\frac{1}{2}}$. The following operator, defined for $z \notin \sigma\left(H_{0}(k, t)\right)$ will appear many times along the paper:

$$
K(z, k, t):=\left|R_{0}(z, k, t)\right|^{\frac{1}{2}} V R_{0}(z, k, t)^{\frac{1}{2}}
$$

The fact that $V$ is $H_{0}(k, t)$-bounded in the form sense with relative bound zero is implied by the following lemma:

Lemma 2.1 : Let $r>-\frac{1}{2}$. Then uniformly in $t$ and $k$

$$
\lim _{a \rightarrow \infty}\left\|K\left(-a^{2}, k, t\right)\right\|=0
$$

As a consequence one can use the KLMN theorem [27] to define $H(k, t)$ as a form sum with form domain $\mathcal{H}^{1}$. The associated quadratic form is

$$
\mathcal{H}^{1} \ni \phi \rightarrow \sum_{n}|(n+k+t) \phi(k, n)|^{2}+\sum_{m, n \in \mathbb{Z}} \hat{V}(m-n) \bar{\phi}(k, m) \phi(k, n)
$$

Due to Lemma 2.1 one can write a useful formula for $R(z, k, t):=(H(k, t)-z)^{-1}$. Indeed, for sufficiently large $a$

$$
R\left(-a^{2}, k, t\right)=R_{0}\left(-a^{2}, k, t\right)^{\frac{1}{2}}\left(1+K\left(-a^{2}, k, t\right)\right)^{-1}\left|R_{0}\left(-a^{2}, k, t\right)\right|^{\frac{1}{2}},
$$

one has by analytic continuation

$$
R(z, k, t)=R_{0}(z, k, t)^{\frac{1}{2}}(1+K(z, k, t))^{-1}\left|R_{0}(z, k, t)\right|^{\frac{1}{2}}
$$

Since $\left|R_{0}(z, k, t)\right|^{\frac{1}{2}}$ is compact, it follows from (2.21) that $R(z, k, t)$ is compact which implies (see [28]) that the spectrum of $H(k, t)$ is discrete. In what concerns the evolution $U(k, s, t)$ generated by $H(k, t)$, as a corollary of Thm. 2.27 in [29] one has the following result assuring the existence of $U(k ; s, t)$ in the weak sense:

Lemma 2.2 Let $\mathcal{H}^{-1}$ denote the space of continuous linear forms on $\mathcal{H}^{1}$. Then for every $\phi(k, 0) \in \mathcal{H}^{1}$ it exists a unique function $\phi(k, \cdot): \mathbb{R} \rightarrow \mathcal{H}^{1}$ such that 
(i) $\mathbb{R} \ni t \rightarrow(\phi(k, t), \psi)$ is continuous for any $\psi \in \mathcal{H}^{-1}$,

(ii) for every $f \in \mathcal{H}^{1}$,

$$
\frac{1}{i} \frac{d}{d t}(\phi(k, t), f)+(\phi(k, t), H(k, t) f)=0 .
$$

(iii) The map $U(k, t, s): \phi(k, s) \rightarrow \phi(k, t)$ is isometric and its extension by continuity is unitary in $l^{2}(\mathbb{Z})$.

From (2.2), (2.7) and Lemma 2.2 we can write the following formula for the StarkWannier evolution

$$
e^{-i(t-s) H^{S W}}=G(t)^{*} S^{*} \int_{[0,1)}^{\oplus} U(k, t, s) d k S G(s),
$$

so the study of $e^{-i(t-s) H^{S W}}$ is reduced to the study of the family of evolutions $U(k, t, s)$ in $l^{2}(\mathbb{Z})$ generated by $H(k, t)$, and the rest of the paper will be devoted to this problem. From (2.8) - (2.12) it follows that $H(k, t)=H(0, k+t)$ and then

$$
U(k, t, s)=U(0, k+t, k+s)
$$

which means that one can restrict the analysis to the fiber $k=0$, and from now on we omit to write $k$ when it is taken to be zero e.g. $H(k=0, t)=H(t), E_{n, 0}(k=$ $0, t)=E_{n, 0}(t)$ etc. The next remark is that although $H(t)$ is not periodic in $t$ there is a hidden periodicity. More precisely if $T$ is the shift operator in $l^{2}(\mathbb{Z})$ :

$$
(T \psi)(n)=\psi(n-1)
$$

then by direct computation one can see that

$$
T H(k, t+1) T^{*}=H(k, t),
$$

which in turn implies

$$
U(t+1, s+1)=T^{*} U(t, s) T
$$

The analysis of $U(t, s)$ can be restricted to one period. We take the basic period to be the union of two half-periods:

$$
t \in I_{0} \cup I_{1} ; \quad I_{0}=\left[-\frac{1}{4}, \frac{1}{4}\right), I_{1}=\left[\frac{1}{4}, \frac{3}{4}\right) .
$$

Let us consider now in more detail the spectral properties of $H_{0}(t)$ and $H(t)$. We remark that when $t \in I_{0} \cup I_{1}, H_{0}(t)$ has degeneracies at the points $t=0$ and $t=\frac{1}{2}$. Actually the splitting of one period into two half-periods is due to the existence of two points per period where $H_{0}(t)$ has degeneracies. Since the latter play a key role in the dynamics (due to the Zener transitions) the above structure suggests to describe $\sigma\left(H_{0}(t)\right)$ inside $I_{l}, l=0,1$ as a union of pairs of eigenvalues that cross at $t=0$ and $t=\frac{1}{2}$. We define (see Fig. 1 ) 


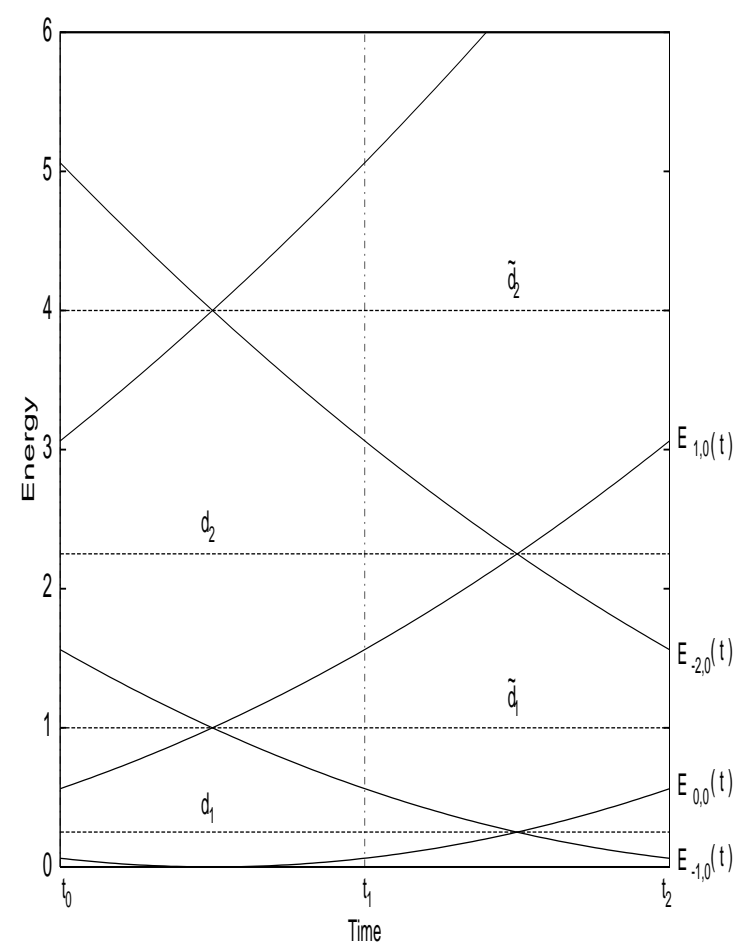

Figure 1: The first eigenvalues of the free Hamiltonian $H_{0}(t)$ as a function of time and the vertical lines $d_{m}, \tilde{d}_{m}$ used in the text.

$$
\begin{aligned}
& \sigma_{m, 0}(t):=E_{m-1,0}(t) \cup E_{-(m-1), 0}(t), \quad \text { for } m \geq 1, \quad t \in I_{0} \\
& \tilde{\sigma}_{m, 0}(t):=E_{m-1,0}(t) \cup E_{-m, 0}(t), \quad \text { for } m \geq 1, \quad t \in I_{1}
\end{aligned}
$$

so that $\sigma\left(H_{0}(t)\right)=\cup_{m \geq 1} \sigma_{m, 0}(t), \sigma\left(H_{0}(t)\right)=\cup_{m \geq 1} \tilde{\sigma}_{m, 0}(t)$ on $I_{0}$ and $I_{1}$ respectively. An alternative way of labelling the eigenvalues of $H_{0}(t)$ is to count them in the increasing order

$$
E_{1}^{0}(t) \leq E_{2}^{0}(t) \leq E_{3}^{0}(t) \ldots \quad t \in \mathbb{R}
$$

Of course the two labellings are related: for a fixed $\alpha$ and a given $t$ there exists an $n_{\alpha}(t)$ such that

$$
E_{\alpha}^{0}(t)=E_{n_{\alpha}(t), 0}(t) \quad \text { and } P_{\alpha}^{0}(t)=P_{n_{\alpha}(t), 0}
$$

Let $t \in\left[-\frac{1}{2}, 0\right)+\frac{N}{2}$ with $N=0,1,2, \ldots$. Then the correspondence between $\alpha$ and $n_{\alpha}(t)$ is given as follows:

$$
n_{\alpha}(t)=\left\{\begin{array}{cl}
\frac{\alpha-N}{2}, & \text { if } N \text { and } \alpha \text { have the same parity } \\
-\frac{\alpha+N-1}{2}, & \text { if } N \text { and } \alpha \text { have different parities. }
\end{array}\right.
$$

With this labelling one has

$$
\begin{aligned}
\sigma_{1,0}(t) & =E_{1}^{0}(t) ; \quad \sigma_{m, 0}(t)=E_{2 m-2}^{0} \cup E_{2 m-1}^{0}, \quad \text { for } t \in I_{0} \\
\tilde{\sigma}_{m, 0}(t) & =E_{2 m-1}^{0} \cup E_{2 m}^{0}, \quad \text { for } t \in I_{1} .
\end{aligned}
$$


The spectral projections of $H_{0}(t)$ corresponding to $\sigma_{m, 0}(t)$ and $\tilde{\sigma}_{m, 0}(t)$ are denoted $Q_{m, 0}$ and $\tilde{Q}_{m, 0}$ :

$$
\begin{array}{ll}
Q_{1,0}=P_{0,0} ; & Q_{m, 0}=P_{m-1,0}+P_{-(m-1), 0} \quad \text { for } t \in I_{0} \\
\tilde{Q}_{m, 0}=P_{m-1,0}+P_{-m, 0} \quad \text { for } t \in I_{1} .
\end{array}
$$

By construction, for any $m=1,2 \ldots$ and $t \in I_{0}, \sigma_{m, 0}(t)$ are well separated . Similarly, for $t \in I_{1} \tilde{\sigma}_{m, 0}(t)$ are well separated:

$$
\begin{aligned}
& \inf _{t \in I_{0}} \operatorname{dist}\left(\sigma_{m, 0}(t), \sigma_{m+1,0}(t)\right)=m-\frac{1}{2} \\
& \inf _{t \in I_{1}} \operatorname{dist}\left(\tilde{\sigma}_{m, 0}(t), \tilde{\sigma}_{m+1,0}(t)\right)=m .
\end{aligned}
$$

Moreover, at the end of the interval $I_{0}$, the two eigenvalues composing $\sigma_{m, 0}(t)$ (with the exception of $\sigma_{1,0}(t)$ which consists in just one eigenvalue) are well separated e.g.

$$
E_{2 m-1}^{0}\left(-\frac{1}{4}\right)-E_{2 m-2}^{0}\left(-\frac{1}{4}\right)=m-1 .
$$

The same is true for the two eigenvalues composing $\tilde{\sigma}_{m, 0}(t)$. We come now to the spectral properties of $H(t)$.

Since $K\left(n+\frac{1}{2}, t\right)$ tends to zero as $n$ tends to infinity, uniformly with respect to $t$ the analytic perturbation theory of type $B$, see [Kato, chap VII], works here as long as $r>-\frac{1}{2}$. More precisely if we label the eigenvalues of $H(t)$ in increasing order $E_{\alpha}(t), \alpha=1, \ldots$ then $\lim _{\alpha \rightarrow \infty}\left(E_{\alpha}(t)-E_{\alpha}^{0}(t)\right)=0$. In addition for $t \neq 0, \frac{1}{2}$, when all the eigenvalues are nondegenerate the corresponding spectral projections $P_{\alpha}(t)$ are close the unperturbed ones: $\lim _{\alpha \rightarrow \infty}\left\|P_{\alpha}(t)-P_{\alpha}^{0}(t)\right\|=0$. In the following $\varphi_{\alpha}(t)$ denotes the eigenfunction of $H(t)$ corresponding to the eigenvalue $E_{\alpha}(t)$.

Let

$$
\Gamma_{m}=\left\{\left(m-\frac{1}{2}\right)^{2}+i y \mid y \in \mathbb{R}\right\}, \quad \tilde{\Gamma}_{m}=\left\{m^{2}+i y \mid y \in \mathbb{R}\right\} \quad m=1,2, \ldots
$$

be vertical lines in the energy plane. In what follows, each time a quantity will be bounded from above by a positive constant, this will be denoted by $C$, while for the lower bounds we denote it $c$.

The main estimates, used many times along the paper are the following:

Lemma 2.3: Let $A<\infty$. Then for each $r>-\frac{1}{2}$ there exists a constant $C_{V}$ independent of $m$ and a positive integer $N_{r}$ such that for any $m>N_{r}$ the following estimate hold:

$$
\begin{aligned}
& \sup _{t \in I_{0}}\left(\sup _{z \in \Gamma_{m}}\|K(z, t)\|+\int_{\Gamma_{m}}\left\|R_{0}(z, t)\right\| \cdot\|K(z, t)\| d y\right) \\
& +\sup _{t \in I_{1}}\left(\sup _{z \in \tilde{\Gamma}_{m}}\|K(z, t)\|+\int_{\tilde{\Gamma}_{m}}\left\|R_{0}(z, t)\right\| \cdot\|K(z, t)\| d y\right) \\
& +\max _{t=-\frac{1}{4}, \frac{1}{4}}\left(\sup _{z \in \Gamma_{m} \cup \tilde{\Gamma}_{m}}\|K(z, t)\|+\int_{\Gamma_{m} \cup \tilde{\Gamma}_{m}}\left\|R_{0}(z, t)\right\| \cdot\|K(z, t)\| d y\right) \\
& \leq C_{V} b(m),
\end{aligned}
$$


where

$$
b(m):=\frac{\log ^{2}\langle 4 m-1\rangle}{\langle m\rangle^{1+\min \{0,2 r\}}} .
$$

Also one has

$$
\lim _{y \rightarrow \infty} \sup _{t \in I_{0}} \sup _{x \leq A}\|K(x+i y, t)\|=0 .
$$

Let $m>1, d_{m}:=\left(m-\frac{1}{2}\right)^{2}, \tilde{d}_{m}:=m^{2}$ and $\gamma_{m}, \tilde{\gamma}_{m}$ be closed finite contours that intersect the real axis in and only in $d_{m}, d_{m-1}$ and $\tilde{d}_{m}, \tilde{d}_{m-1}$ (for example $\gamma_{m}$ can be a square of length $8(m-1))$. Then there exist an absolute constant $C$ such that:

$$
\begin{aligned}
& \sup _{t \in I_{0}} \sup _{z \in \gamma_{m}}\|K(z, t)\| \leq C\left\|K\left(d_{m}, 0\right)\right\|, \\
& \sup _{t \in I_{1}} \sup _{z \in \tilde{\gamma}_{m}}\|K(z, t)\| \leq C\left\|K\left(\tilde{d}_{m}, 0\right)\right\| .
\end{aligned}
$$

Due to the above lemma we are able to give upper bounds of the type $\left(C_{V}\langle b(m)\rangle\right)^{N}$. In the following we shall say that a quantity $A$ is of order $\left(\mathcal{O}_{r}(\langle b(m)\rangle)^{N}\right)$ if it obeys the estimate $\|A\| \leq\left(C_{V}\langle b(m)\rangle\right)^{N}$. The subscript $r$ reminds us the dependence on $\|V\|_{r}$. We notice that the third line in (2.41) follows at once from the estimates of the first and the second lines. As we have said, the proof of the Lemma is postponed to Appendix 7.1.

Now we are ready to spell the spectral properties of $H(t)$. We shall consider only $t \in I_{0}$; the results and the proofs for $t \in I_{1}$, with the appropiate identifications, are the same. The first remark is that there exists an $m^{*}$ such that for any $m>m^{*}$ the norm of $K(z, t)$ is smaller than $\frac{1}{2}$. Secondly, for all $t \in I_{0} d_{m} \in \rho(H(t))$ and for all $t \in I_{1} \tilde{d}_{m} \in \rho(H(t))$. Moreover, $\tilde{d}_{m} \in \rho\left(H\left(-\frac{1}{4}\right)\right)$ :

Corollary 2.4: Let $m>m^{*}$. Then

$$
\min \left\{\inf _{t \in I_{0}} \operatorname{dist}\left(d_{m}, \sigma(H(t))\right), \inf _{t \in I_{1}} \operatorname{dist}\left(\tilde{d}_{m}, \sigma(H(t))\right)\right\} \geq \mathrm{cm} .
$$

Let $Q_{m}(t)$ the spectral projection associated to $\sigma_{m}(t)=\left(d_{m-1}, d_{m}\right) \cap \sigma(H(t))$. Then one has the following:

Corollary 2.5: For $m$ sufficiently large or $V$ small enough

$$
\sup _{t \in I_{0}}\left\|Q_{m}(t)-Q_{m, 0}\right\| \leq C_{V} b(m)
$$

Let for $\alpha=2 m-2,2 m-1($ see (2.33) $)$

$$
P_{\alpha}^{0}\left(-\frac{1}{4}\right)=P_{n_{\alpha}\left(-\frac{1}{4}\right), 0} ; \quad \varphi_{\alpha}^{0}\left(-\frac{1}{4}\right)=\varphi_{n_{\alpha}\left(-\frac{1}{4}\right), 0},
$$

and $P_{2 m-2}\left(-\frac{1}{4}\right), P_{2 m-1}\left(-\frac{1}{4}\right)$ the spectral projections of $H\left(-\frac{1}{4}\right)$ corresponding to the intervals $\left(d_{m-1}, \tilde{d}_{m-1}\right)$ and $\left(\tilde{d}_{m-1}, d_{m}\right)$ respectively. Then the following estimate holds: 
Corollary 2.6: Let $\alpha=2 m-2,2 m-1$. Then for $m>m^{*}$

$$
\left\|P_{\alpha}\left(-\frac{1}{4}\right)-P_{\alpha}^{0}\left(-\frac{1}{4}\right)\right\| \leq C_{V} b(m) .
$$

For sufficiently large $m$, by Sz-Nagÿ lemma (see Appendix 7.2), $P_{\alpha}\left(-\frac{1}{4}\right)$ and $P_{\alpha}^{0}\left(-\frac{1}{4}\right)$ are unitarily equivalent so that $H\left(-\frac{1}{4}\right)$ has nondegenerate eigenvalues in $\left(d_{m-1}, \tilde{d}_{m-1}\right)$ and $\left(\tilde{d}_{m-1}, d_{m}\right)$ respectively. They are denoted by $E_{2 m-2}\left(-\frac{1}{4}\right)$ and $E_{2 m-1}\left(-\frac{1}{4}\right)$. Moreover, if $N_{\alpha}\left(-\frac{1}{4}\right)$ is the Sz-Nagÿ transformation corresponding to the pair $P_{\alpha}\left(-\frac{1}{4}\right), P_{\alpha}^{0}\left(-\frac{1}{4}\right)$ then

$$
\varphi_{\alpha}\left(-\frac{1}{4}\right)=N_{\alpha}\left(-\frac{1}{4}\right) \varphi_{\alpha}^{0}\left(-\frac{1}{4}\right)
$$

are eigenvectors of $H\left(-\frac{1}{4}\right)$ corresponding to $E_{\alpha}\left(-\frac{1}{4}\right)$. We say that an eigenbasis $\left\{\varphi_{\alpha}\left(-\frac{1}{4}\right)\right\}_{\alpha=1}^{\infty}$ of $H\left(-\frac{1}{4}\right)$ is a canonical one if for sufficiently large $\alpha$ (such that all the above construction works) $\varphi_{\alpha}\left(-\frac{1}{4}\right)$ is given by (2.50). In the same way one constructs canonical bases for $t=\frac{1}{4}$. For $t_{l}:=-\frac{1}{4}+\frac{l}{2}, l=2,3 \ldots$ the canonical bases are provided by (see (2.26) $)$

$$
T \varphi_{\alpha}\left(t_{l}+1\right)=\varphi_{\alpha}\left(t_{l}\right)
$$

Before going to the proofs let us write the reduction to one period formula (2.27) in the canonical basis.

Lemma 2.7 Let $l$ be even, $t_{0}=-\frac{1}{4}, t_{l}=t_{0}+\frac{l}{2}$. Then for $\alpha$ sufficiently large

$$
\left\langle\varphi_{\alpha}\left(t_{l}+1\right), U\left(t_{l}+1, t_{l}\right) \varphi_{\beta}\left(t_{l}\right)\right\rangle=\left\langle\varphi_{\alpha}\left(t_{2}\right), U\left(t_{2}, t_{0}\right) \varphi_{\beta}\left(t_{0}\right)\right\rangle .
$$

Now we present the proofs for the lemmae and corollaries given above:

Proof of Lemma 2.1: We estimate the Hilbert-Schmidt norm of $K\left(-a^{2}, k, t\right)$

$$
\begin{aligned}
\left\|K\left(-a^{2}, k, t\right)\right\|^{2} & \leq\left\|K\left(-a^{2}, k, t\right)\right\|_{H S}^{2} \\
& =\sum_{m, n \in \mathbb{Z}}\left|\frac{1}{\sqrt{(m+k+t)^{2}+a^{2}}} \hat{V}(m-n) \frac{1}{\sqrt{(n+k+t)^{2}+a^{2}}}\right|^{2} \\
& \leq\|V\|_{r}^{2} \sum_{m, n \in \mathbb{Z}} \frac{1}{(m+k+t)^{2}+a^{2}}\langle m-n\rangle^{-2 r} \frac{1}{(n+k+t)^{2}+a^{2}} \\
& \leq\|V\|_{r}^{2}\left(\frac{\sqrt{5}}{2}\right)^{\max \{-2 r, 0\}} \sum_{m \in \mathbb{Z}} \frac{\langle m\rangle^{\max \{-2 r, 0\}}}{(m+k+t)^{2}+a^{2}},
\end{aligned}
$$

where in the last line we used the inequality

$$
\langle m-n\rangle^{-2 r} \leq\left(\frac{\sqrt{5}}{2}\langle m\rangle\langle n\rangle\right)^{\max \{-2 r, 0\}}
$$


Then the above series is convergent for $r>-\frac{1}{2}$ and the lemma is proved using the Lebesque dominated convergence theorem.

Proof of Corollary 2.4: $R\left(d_{m}, t\right)$ can be written as

$$
R\left(d_{m}, t\right)=\left|R_{0}\left(d_{m}, t\right)\right|^{\frac{1}{2}}\left(1+K\left(d_{m}, t\right)\right)^{-1} R_{0}\left(d_{m}, t\right)^{\frac{1}{2}} .
$$

Taking into account that $\operatorname{dist}\left(d_{m}, \sigma\left(H_{0}(t)\right)\right) \geq \mathrm{cm}$ one obtains from (2.54)

$$
\left\|R\left(d_{m}, t\right)\right\| \leq 2\left\|\left|R_{0}\left(d_{m}, t\right)\right|^{\frac{1}{2}}\right\| \cdot\left\|R_{0}\left(d_{m}, t\right)^{\frac{1}{2}}\right\|=2\left\|R_{0}\left(d_{m}, t\right)\right\| \leq 2(C m)^{-1},
$$

which implies $\inf _{t \in I_{0}} \operatorname{dist}\left(d_{m}, \sigma(H(t))\right) \geq \mathrm{cm}$. The proof of the inequality $\operatorname{dist}\left(\tilde{d}_{m}, \sigma\left(H\left(-\frac{1}{4}\right)\right) \geq C m / 2\right.$ is similar.

Proof of Corollary 2.5: By perturbation theory

$$
\begin{aligned}
Q_{m}(t)-Q_{m, 0} & =\frac{i}{2 \pi} \oint_{\gamma_{m}}\left(R(z, t)-R_{0}(z, t)\right) d z \\
& =-\frac{i}{2 \pi} \int_{\gamma_{m}} R_{0}(z, t)^{\frac{1}{2}} K(z, t)(1+K(z, t))^{-1}\left|R_{0}(z, t)\right|^{\frac{1}{2}} d z .
\end{aligned}
$$

Taking the norms, using that $\oint_{\gamma_{m}}\left\|R_{0}(z, t)\right\| d z \leq C$ the estimate (2.47) follows from (2.44) and (2.41).

Proof of Corollary 2.6: Similar to the proof of Corollary 2.5.

Proof of Lemma 2.7: Direct computation using (2.27), (2.50) and (2.51).

\section{The adiabatic theorem}

As already said in the introduction our first task is to show that at high energies the most important "interband transitions" during a half Bloch period are the so-called Zener transitions, namely the ones between neighbouring bands (e.g on $I_{0}$ between $E_{2 m-2}(t)$ and $\left.E_{2 m-1}(t)\right)$. This is nothing else but to claim that for $t, s \in I_{0}$ the subspace $Q_{m}(t) \mathcal{H}$ is almost invariant under the evolution $U(t, s)$. In this section we construct adiabatic evolutions $U_{m}^{A}\left(t, t_{0}\right)$ and $\tilde{U}_{m}^{A}\left(t, t_{1}\right)$ on $I_{0}$ and $I_{1}$ respectively, satisfying

$$
\begin{aligned}
U_{m}^{A}\left(t, t_{0}\right) Q_{m}\left(t_{0}\right) \mathcal{H} & =Q_{m}(t) \mathcal{H}, & & t \in I_{0} \\
\tilde{U}_{m}^{A}\left(t, t_{1}\right) \tilde{Q}_{m}\left(t_{1}\right) \mathcal{H} & =\tilde{Q}_{m}(t) \mathcal{H}, & & t \in I_{1}
\end{aligned}
$$

and prove that they are close to $U\left(t, t_{0}\right)$ and $U\left(t, t_{1}\right)$ respectively. We consider first the half Bloch period $I_{0}$ and at the end indicate the changes for $I_{1}$. Let

$$
Q_{m}^{+}(t):=\sum_{j=m+1}^{\infty} Q_{j}(t), \quad Q_{m}^{-}(t):=\sum_{j=1}^{m-1} Q_{j}(t), \quad t \in I_{0}
$$


and define $H_{m}^{A}(t)$ by

$$
\begin{aligned}
H_{m}^{A}(t) & :=H(t)-X_{m}(t) \\
& =: H(t)-i Q_{m}^{+}(t) \dot{Q}_{m}^{+}(t)-i Q_{m}(t) \dot{Q}_{m}(t)-i Q_{m}^{-}(t) \dot{Q}_{m}^{-}(t) .
\end{aligned}
$$

It turns out (see the proof of Lemma 3.4 below) that $X_{m}(t)$ are bounded hence $H_{m}^{A}(t)$ are self-adjoint on $\mathcal{D}(H(t))$. The adiabatic evolution $U_{m}^{A}\left(t, t_{0}\right)$ generated by $H_{m}^{A}(t)$ will be defined as the solution (in the weak sense, as in Lemma 2.2 ) of the equation

$$
i \frac{d}{d t} U_{m}^{A}\left(t, t_{0}\right)=H_{m}^{A}(t) U_{m}^{A}\left(t, t_{0}\right), \quad U_{m}^{A}\left(t_{0}, t_{0}\right)=1 .
$$

As in the case of standard adiabatic theorem of quantum mechanics, $U_{m}^{A}\left(t, t_{0}\right)$ has the intertwining property

$$
Q_{m}(t)=U_{m}^{A}\left(t, t_{0}\right) Q_{m}\left(t_{0}\right) U_{m}^{A}\left(t, t_{0}\right)^{*}, \quad Q_{m}^{ \pm}(t)=U_{m}^{A}\left(t, t_{0}\right) Q_{m}^{ \pm}\left(t_{0}\right) U_{m}^{A}\left(t, t_{0}\right)^{*}
$$

i.e the families of subspaces $Q_{m}(t) \mathcal{H}, Q_{m}^{ \pm}(t) \mathcal{H}$ are invariant under $U_{m}^{A}\left(t, t_{0}\right)$. The proof is standard ( Krein-Kato Lemma [25, 18, 15]) For $t \in I_{1}$ one constructs in a similar way $\tilde{U}_{m}^{A}\left(t, t_{1}\right)$ by replacing $Q_{j}(t)$ by $\tilde{Q}_{j}(t)$. The main result of this section is to show that $U_{m}^{A}\left(t, t_{0}\right)$ and $U\left(t, t_{0}\right)$ are close:

Theorem 3.1 For any $r>-\frac{1}{2}$ and $m$ sufficiently large or $\|V\|_{r}$ small enough it holds:

$$
\begin{aligned}
& \sup _{t \in I_{0}}\left\|U\left(t, t_{0}\right)-U_{m}^{A}\left(t, t_{0}\right)\right\| \leq C_{V} b(m)\langle m\rangle^{-1}, \\
& \sup _{t \in I_{1}}\left\|U\left(t, t_{1}\right)-\tilde{U}_{m}^{A}\left(t, t_{1}\right)\right\| \leq C_{V} b(m)\langle m\rangle^{-1} .
\end{aligned}
$$

where $C_{V}$ is a constant that depends on $V$ and $b(m)$ was introduced in Lemma 2.3.

The basic steps of the proof are the standard ones but we have to check the relevant estimates. Define

$$
\Omega_{m}\left(t, t_{0}\right):=U_{m}^{A}\left(t, t_{0}\right)^{*} U\left(t, t_{0}\right),
$$

and notice that $\left\|\Omega_{m}\left(t, t_{0}\right)-1\right\|=\left\|U_{m}^{A}\left(t, t_{0}\right)-U\left(t, t_{0}\right)\right\|$, so one is left with the estimation of $\left\|\Omega_{m}\left(t, t_{0}\right)-1\right\|$. Since a direct estimation of

$$
i\left(\Omega_{m}\left(t, t_{0}\right)-1\right)=\int_{t_{0}}^{t} d s U_{m}^{A}\left(s, t_{0}\right)^{*} X_{m}(s) U_{m}^{A}\left(s, t_{0}\right) \Omega_{m}\left(s, t_{0}\right)
$$

would give (see Lemma 3.4) a bound of order $\mathcal{O}_{r}(b(m))$, which is not accurate enough for the error estimates in the long time behaviour we have to follow the proof of the standard adiabatic theorem and make "an integration by parts". More precisely we have: 
Lemma 3.2: $X_{m}(t)$ can be written in the form (we omit for simplicity the timedependence):

$$
X_{m}=\left[H, Y_{m}\right]
$$

with:

$$
\begin{aligned}
Y_{m} & =\frac{1}{2 \pi} \oint_{\gamma_{m}^{-}} d z R(z)\left\{Q_{m}^{-} \dot{Q}_{m}^{-}-Q_{m}^{+} \dot{Q}_{m}^{+} Q_{m}^{-}\right\} R(z) \\
& +\frac{1}{2 \pi} \oint_{\gamma_{m}} d z R(z)\left\{Q_{m} \dot{Q}_{m}-Q_{m}^{+} \dot{Q}_{m}^{+} Q_{m}\right\} R(z)
\end{aligned}
$$

Here $\gamma_{m}^{-}$is a closed contour around $\sigma\left(Q_{m}^{-} H(t)\right)$. Now the integration by parts will give $\left\|\Omega_{m}\left(t, t_{0}\right)-1\right\|$ in terms of $Y_{m}$ :

Lemma 3.3: If $t$ belongs to $I_{0}$ the following estimate holds:

$$
\left.\| \Omega_{m}\left(t, t_{0}\right)-1\right)\|\leq\| Y_{m}(t)\|+\| Y_{m}\left(t_{0}\right) \|+\int_{t_{0}}^{t} d s\left(\left\|X_{m}(s) Y_{m}(s)\right\|+\left\|\dot{Y}_{m}(s)\right\|\right)
$$

so we are left with verifying (3.11, 3.13) and to estimate the norms in (3.13). The basic estimation is contained in:

Lemma 3.4: For $m$ sufficiently large

$$
\sup _{t \in I_{0}}\left(\left\|\dot{Q}_{m}(t)\right\|+\left\|\dot{Q}_{m}^{-}(t)\right\|+\left\|\dot{Q}_{m}^{+}(t)\right\|\right) \leq C_{V} b(m)
$$

Notice that since $Q_{m}^{+}(t)=1-Q_{m}(t)-Q_{m}^{-}(t)$ we have only to estimate $\left\|\dot{Q}_{m}(t)\right\|$ and $\left\|\dot{Q}_{m}^{-}(t)\right\|$. Taking Lemma 3.4 for granted we give now the proofs of Lemmae 3.2 and 3.3 .

Proof of Lemma 3.2: Since by Lemma $3.4 X_{m}(t)$ is bounded

$$
\sup _{t \in I_{0}}\left\|X_{m}(t)\right\| \leq C_{b}(m)
$$

and in addition (remember that for an orthogonal projection valued function $E(t)$ $E(t) \dot{E}(t) E(t)=0) X_{m}(t)$ is off-diagonal with respect to the decomposition $\mathcal{H}=$ $Q_{m}^{+}(t) \mathcal{H} \oplus Q_{m} \mathcal{H} \oplus Q_{m}^{-} \mathcal{H}$ one obtains $Y_{m}(t)$ as the solution of the commutator equation (3.11) by using Theorem 9.3 in [11. Actually one can verify that (3.12) solves 3.11) by straightforward computation.

Proof of Lemma 3.3: Using the identity (we omit for simplicity the index $m$ and the time-dependence):

$$
\begin{aligned}
i \frac{d}{d t}\left(U^{A^{*}} Y U^{A}\right) & =U^{A^{*}}\left\{i \dot{Y}-\left[H^{A}, Y\right]\right\} U^{A} \\
& =U^{A^{*}}\{i \dot{Y}-X+[X, Y]\} U^{A}
\end{aligned}
$$


and replacing $U^{A^{*}} X U^{A}$ in (3.10) one has:

$$
\begin{aligned}
& i(\Omega-1)=\int_{t_{0}}^{t} d s\left\{-i \frac{d}{d s}\left(U^{A^{*}} Y U^{A}\right)+U^{A^{*}}[X, Y] U^{A}+i U^{A^{*}} \dot{Y} U^{A}\right\} \Omega \\
& =\int_{t_{0}}^{t} d s\left\{-i \frac{d}{d s}\left(U^{A^{*}} Y U^{A} \Omega\right)+i U^{A^{*}} Y U^{A} \dot{\Omega}+U^{A^{*}}[X, Y] U^{A} \Omega+i U^{A^{*}} \dot{Y} U^{A} \Omega\right\} \\
& =-\left.i U^{A^{*}} Y U^{A} \Omega\right|_{t_{0}} ^{t}+\int_{t_{0}}^{t} d s U^{A^{*}}\{X Y+i \dot{Y}\} U^{A} \Omega
\end{aligned}
$$

which in norm gives the claimed result.

Proof of Lemma 3.4: We give detailed calculations only for $\dot{Q}_{m}^{-}(t)$. Computing $Q_{m}$ perturbatively, using the identity $\dot{R}(z, t)=-R(z, t) \dot{H}(t) R(z, t)$ one has

$$
\begin{aligned}
\dot{Q}_{m}^{-}(t) & =\frac{d}{d t}\left\{Q_{m, 0}^{-}-\frac{i}{2 \pi} \oint_{\gamma_{m}^{-}} d z R_{0}(z) V R(z)\right\} \\
& =\frac{i}{2 \pi} \oint_{\gamma_{m}^{-}} d z\left\{R_{0}(z) \dot{H}_{0} R_{0}(z) V R(z)+R_{0}(z) V R(z) \dot{H} R(z)\right\}
\end{aligned}
$$

where we omited the explicit $t$-dependence. To go further with the estimates we deform first $\gamma_{m}^{-}$to $\gamma_{m}^{-}(L):=\gamma_{m-1}(L) \cup \gamma_{m-1}^{\prime}(L) \cup \gamma_{m-1}^{\prime \prime}(L) \cup \gamma_{0}(L)$ with:

$$
\begin{aligned}
\gamma_{m-1}(L) & :=\left\{d_{m-1}+i y \mid y \in[-L, L]\right\} \\
\gamma_{m-1}^{\prime}(L) & :=\left\{x+i L \mid x \in\left[-L, d_{m-1}\right]\right\} \\
\gamma_{m-1}^{\prime \prime}(L) & :=\left\{x-i L \mid x \in\left[-L, d_{m-1}\right]\right\} \\
\gamma_{0}(L) & :=\{-L+i y \mid y \in[-L, L]\},
\end{aligned}
$$

and then use the estimate (2.43) to show that the only integral that survives from $\gamma_{m}^{-}$in the limit $L \rightarrow \infty$ is the one on $\Gamma_{m-1}$ (notice that $\left.\lim _{L \rightarrow \infty} \gamma_{m-1}(L)=\Gamma_{m-1}\right)$. Using the polar decomposition of $R_{0}(z)$ and the resolvent equation for $R(z)$ it follows that

$$
\begin{aligned}
\left\|\dot{Q}_{m}^{-}(t)\right\| & \leq \frac{1}{2 \pi} \oint_{\Gamma_{m-1}}|d z|\left\|R_{0}(z)\right\|\|K(z)\| \cdot\left\|\left|R_{0}(z)\right|^{\frac{1}{2}} \dot{H}_{0} R_{0}(z)^{\frac{1}{2}}\right\| \cdot\left\|(1+K(z))^{-1}\right\| \\
& +\frac{1}{2 \pi} \oint_{\Gamma_{m-1}}|d z|\left\|R_{0}(z)\right\|\|K(z)\| \cdot\left\|\left|R_{0}(z)\right|^{\frac{1}{2}} \dot{H}_{0} R_{0}(z)^{\frac{1}{2}}\right\| \cdot\left\|(1+K(z))^{-1}\right\|^{2} .
\end{aligned}
$$

We note that the quantity $\left|R_{0}(z, t)^{\frac{1}{2}}\right| \dot{H}_{0}(t) R_{0}(z, t)^{\frac{1}{2}}$ is uniformly bounded on $\Gamma_{m-1}$ (namely this quantity is bounded in norm by a constant). Indeed:

$$
\begin{aligned}
\sup _{t \in I_{0}} \sup _{z \in \Gamma_{m-1}}\left\|R_{0}(z, t)^{\frac{1}{2}} \dot{H}_{0}(t)\left|R_{0}(z, t)\right|^{\frac{1}{2}}\right\| & =\sup _{t \in I_{0}} \sup _{y \in \mathbb{R}} \sup _{m^{\prime} \in \mathbb{Z}}\left|\frac{2\left(m^{\prime}+t\right)}{\left(m^{\prime}+t\right)^{2}-d_{m-1}-i y}\right| \\
& \leq C .
\end{aligned}
$$

Then using Lemma 2.3 and the inequality $\|(1+K)\|^{-1} \leq(1-\|K\|)^{-1}$ one obtains

$$
\sup _{t \in I_{0}}\left\|\dot{Q}_{m}^{-}(t)\right\| \leq C_{V} b(m-1) .
$$


Since $b(m-1) \leq C b(m)$ we proved the claimed result on $\dot{Q}_{m}^{-}(t)$. The estimate of $\dot{Q}_{m}(t)$ goes along the same line, by deforming the closed contour around $\sigma\left(Q_{m}(t) H\right)$, $\gamma_{m}$, to $\Gamma_{m-1} \cup \Gamma_{m}$. The lemma is now proved

We turn now to the estimations of the norms in the r.h.s of (3.13). For $X_{m}(t)$ Lemma 3.4 gives:

$$
\sup _{t \in I_{0}}\left\|X_{m}(t)\right\| \leq C_{V} b(m),
$$

while for $Y_{m}(t)$ we write

$$
\begin{aligned}
\left\|Y_{m}(t)\right\| & \leq \frac{1}{2 \pi}\left(\left\|\dot{Q}_{m}^{-}(t)\right\|+\left\|\dot{Q}_{m}^{+}(t)\right\|\right) \int_{\Gamma_{m-1}} d z\|R(z, t)\|^{2} \\
& +\frac{1}{2 \pi}\left(\left\|\dot{Q}_{m}(t)\right\|+\left\|\dot{Q}_{m}^{+}(t)\right\|\right) \int_{\Gamma_{m-1} \cup \Gamma_{m}} d z\|R(z, t)\|^{2} .
\end{aligned}
$$

Now for example (use again the estimate of $K(z, t)$ on $\Gamma_{m-1} \cup \Gamma_{m}$ )

$$
\begin{aligned}
& \sup _{t \in I_{0}} \int_{\Gamma_{m-1} \cup \Gamma_{m}}|d z|\|R(z, t)\|^{2} \leq C \sup _{t \in I_{0}} \int_{\Gamma_{m-1} \cup \Gamma_{m}}|d z|\left\|R_{0}(z, t)\right\|^{2} \\
= & \sup _{t \in I_{0}} \sup _{m^{\prime} \in \mathbb{Z}} \int_{-\infty}^{\infty}\left\{\frac{d y}{\left[E_{m^{\prime}, 0}(t)-d_{m-1}\right]^{2}+y^{2}}+\frac{d y}{\left[E_{m^{\prime}, 0}(t)-d_{m}\right]^{2}+y^{2}}\right\} \leq C\langle m\rangle^{-1},
\end{aligned}
$$

which together with Lemma 3.4 gives

$$
\sup _{t \in I_{0}}\left\|Y_{m}(t)\right\| \leq C_{V} b(m)\langle m\rangle^{-1} .
$$

Obviously, $Y_{m}\left(t_{0}\right)$ satisfies the same estimate. To estimate $\left\|\dot{Y}_{m}(t)\right\|$ we observe first that it involves estimates on $\ddot{Q}_{m}^{-}(t), \ddot{Q}_{m}^{+}(t)$ and $\ddot{Q}_{m}(t)$. Indeed, a long but straightforward calculation gives (we omit the index $m$ in $Y_{m}$ ):

$$
\begin{aligned}
\dot{Y}(t) & =-\frac{1}{2 \pi i} \int_{\Gamma_{m-1}} d z\left\{\dot{R}(z, t)\left(Q^{-}(t) \dot{Q}^{-}(t)-Q^{+}(t) \dot{Q}^{+} Q^{-}\right) R(z, t)\right. \\
& +R(z, t)\left(\left(\dot{Q}^{-}(t)\right)^{2}+Q^{-}(t) \ddot{Q}^{-}(t)-\left(\dot{Q}^{+}(t)\right)^{2} Q^{-}(t)\right. \\
& \left.-Q^{+}(t) \ddot{Q}^{+}(t) Q^{-}(t)-Q^{+}(t) \dot{Q}^{+}(t) \dot{Q}^{-}(t)\right) R(z, t) \\
& \left.+R(z, t)\left(Q^{-}(t) \dot{Q}^{-}(t)-Q^{+}(t) \dot{Q}^{+} Q^{-}(t)\right) \dot{R}(z, t)\right\} \\
& -\frac{1}{2 \pi i} \int_{\Gamma_{m-1} \cup \Gamma_{m}} d z\left\{\dot{R}(z, t)\left(Q(t) \dot{Q}(t)-Q^{+}(t) \dot{Q}^{+}(t) Q(t)\right) R(z, t)\right. \\
& +R(z, t)\left(\dot{Q}(t)^{2}+Q(t) \ddot{Q}(t)-\dot{Q}^{+}(t)^{2} Q(t)\right. \\
& \left.-Q^{+}(t) \ddot{Q}^{+}(t) Q(t)-Q^{+}(t) \dot{Q}^{+}(t) \dot{Q}(t)\right) R(z, t) \\
& \left.+R(z, t)\left(Q(t) \dot{Q}(t)-Q^{+}(t) \dot{Q}^{+}(t) Q(t)\right) \dot{R}(z, t)\right\} .
\end{aligned}
$$


In what concerns $\ddot{Q}_{m}^{-}(t)$, one has only to perform all the derivatives involved and then to estimate the terms

$$
\begin{aligned}
\ddot{Q}_{m}^{-}(t) & =\frac{1}{2 \pi i} \int_{\Gamma_{m-1}} d z\left\{R_{0}(z, t) \dot{H}_{0}(t) R_{0}(z, t) \dot{H}_{0}(t) R_{0}(z, t) V R(z, t)\right. \\
& -R_{0}(z, t) \ddot{H}_{0}(t) R_{0}(z, t) V R(z, t) \\
& +R_{0}(z, t) \dot{H}_{0}(t) R_{0}(z, t) \dot{H}_{0}(t) R_{0}(z, t) V R(z, t) \\
& +R_{0}(z, t) \dot{H}_{0}(t) R_{0}(z, t) V R(z, t) \dot{H}_{0} R(z, t) \\
& +R_{0}(z, t) \dot{H}_{0}(t) R_{0}(z, t) V R(z, t) \dot{H}_{0}(t) R(z, t) \\
& +R_{0}(z, t) V R(z, t) \dot{H}_{0}(t) R(z, t) \dot{H}_{0}(t) R(z, t) \\
& -R_{0}(z, t) V R(z, t) \ddot{H}_{0}(t) R(z, t) \\
& \left.+R_{0}(z, t) V R(z, t) \dot{H}_{0}(t) R(z, t) \dot{H}_{0}(t) R(z, t)\right\}
\end{aligned}
$$

We claim that the estimates for these terms involve only powers of $(1+K(z, t))^{-1}$, $R_{0}(z, t)^{\frac{1}{2}} \dot{H}_{0}(t)\left|R_{0}(z, t)\right|^{\frac{1}{2}}$ and the integral of $\left\|R_{0}(z, t)^{\frac{1}{2}}\right\|^{2} \cdot\|K(z, t)\|$ on $\Gamma_{m-1}$ which was already estimated in Lemma 2.3. The result is

$$
\sup _{t \in I_{0}}\left\|\ddot{Q}_{m}^{-}(t)\right\| \leq C_{V} b(m)
$$

The same estimate is satisfied by $\ddot{Q}_{m}(t)$ and then

$$
\sup _{t \in I_{0}}\|\dot{Y}(t)\| \leq C_{V} b(m)\langle m\rangle^{-1} .
$$

The last term to be considered is $X_{m}(t) Y_{m}(t)$ which gives a better estimate

$$
\sup _{t \in I_{0}}\left\|X_{m}(t) Y_{m}(t)\right\| \leq C_{V}^{2} b(m)^{2}\langle m\rangle^{-1} .
$$

Collecting (3.13), (3.21), (3.23), (3.25) and (3.26) one obtains the first estimate from Theorem 3.1. For $\tilde{U}_{m}^{A}\left(t, t_{0}\right)$ the computations and estimations are similar as above, the only difference being that the contour of integration $\tilde{\Gamma}_{m}$ is slightly different, i.e it is shifted upwards with $\frac{1}{2}$ with respect to $\Gamma_{m}$. As a consequence all the estimations will be improved with $\frac{1}{2}$, in the sense that $b(m) \rightarrow b\left(m+\frac{1}{2}\right)$. Thus there is no loss if we write

$$
\sup _{t \in I_{1}}\left\|U\left(t, t_{0}\right)-\tilde{U}_{m}^{A}\left(t, t_{0}\right)\right\| \leq C_{V} b(m)\langle m\rangle^{-1}
$$

The proof of Thm 3.1 is done.

\section{The adiabatic evolution. The effective Hamil- tonian}

As shown in Theorem 3.1 the true evolution over the half-periods $I_{0}$ and $I_{1}$ is well approximated, in the limit of large $m$, by the adiabatic evolutions $U_{m}^{A}$ and $\tilde{U}_{m}^{A}$. 
The next step is to compute, up to small controlable errors, $U_{m}^{A}$ when restricted to $Q_{m}(t) \mathcal{H}$ and $\tilde{U}_{m}^{A}$ when restricted to $\tilde{Q}_{m}(t) \mathcal{H}$. In what follows we consider explicitly only $U_{m}^{A}$ over $I_{0}$ and give the similar results for $\tilde{U}_{m}^{A}$ over $I_{1}$.

Before entering the computation of $U_{m}^{A}$ let us show that, when restricted to $Q_{m}(t) \mathcal{H}, U_{m}^{A}$ is nothing but the simplified dynamics in 2] obtained by keeping only the couplings between almost touching bands. Indeed, consider an initial wave function $\psi_{m}(s) \in Q_{m}(s) \mathcal{H}, s \in I_{0}$. Then, as far as $t \in I_{0}$, the adiabatic vector $\psi_{m}^{A}(t):=U_{m}^{A}(t, s) \psi_{m}(s) \in Q_{m}(t) \mathcal{H}$ and it can be written as

$$
\psi_{m}^{A}(t)=\sum_{j=1}^{2} c_{j}(t) \varphi_{\alpha_{j}}(t), \quad \alpha_{1}=2 m-2, \alpha_{2}=2 m-1,
$$

where $\varphi_{\alpha_{j}}(t)$ are eigenfunctions of $H(t)$ corresponding to the eigenvalues from $\sigma(H(t))$ (one can assume that for $t=t_{0}$ or $t=t_{1}, \varphi_{\alpha_{j}}(t)$ coincide with the canonical eigenfunctions constructed in Section 2). Plugging the decomposition (4.1) into the Schrödinger equation for $\psi_{m}^{A}(t)$ (see Section 3)

$$
i \frac{d}{d t} \psi_{m}^{A}(t)=H_{m}^{A}(t) \psi_{m}^{A}(t)=\left(H(t)-X_{m}(t)\right) \psi_{m}^{A}(t)
$$

taking the scalar product with $\varphi_{\alpha_{l}}(t)$ and using the fact that $X_{m}(t)$ is off-diagonal i.e $Q_{m}(t) X_{m}(t) Q_{m}(t)=0$, one obtains:

$$
i \frac{d}{d t} c_{l}(t)=c_{l}(t) E_{\alpha_{l}}(t)+\sum_{j=1}^{2} \chi_{l, j}(t) c_{j}(t)
$$

where

$$
\chi_{l, j}(t):=-i\left\langle\varphi_{\alpha_{l}}(t), \frac{d}{d t} \varphi_{\alpha_{j}}(t)\right\rangle
$$

which is nothing but the equation given in Ao's paper.

To compute $U_{m}^{A}$ amounts then to solve the $2 \times 2$ system (4.3) and this problem has been considered many times (see [2, 12, 19] and references therein) in the physical literature. The trouble with (4.3) is that $\chi_{l, j}(t)$ are not easy to compute up to a controled error in the limit $m \rightarrow \infty$ since due to the existence of quasi-crossings one has to deal with almost degenerate perturbation theory. To our knowledge the earlier papers take for $\chi_{l, j}(t)$ a low order perturbation theory formula, but never controlled the rest. We avoid this difficulty by using the reduction theory which is the standard tool in analytic perturbation theory [15] and was as well extended to other contexts, in particular for adiabatic perturbation theory (see e.g [25, 21] and references therein). More precisely, for $m$ sufficiently large (see Corollary 2.2) $\left\|Q_{m}(t)-Q_{m, 0}\right\|<1$ and then (see Appendix 7.2) one can write the Sz-Nagÿ transformation matrix corresponding to the pair $Q_{m}(t), Q_{m, 0}$ :

$$
Q_{m}(t)=W_{m}(t) Q_{m, 0} W_{m}(t)^{*} .
$$

Then if one defines for $t, s \in I_{0}$ :

$$
\mathcal{U}_{m}(t, s):=W_{m}(t)^{*} U_{m}^{A}(t, s) W_{m}(s)
$$


it can be checked by straightforward calculation that

$$
i \frac{d \mathcal{U}_{m}(t, s)}{d t}=\left(i \dot{W}_{m}(t)^{*} W_{m}(t)+W_{m}(t)^{*} H_{m}^{A}(t) W_{m}(t)\right) \mathcal{U}_{m}(t, s)
$$

and that

$$
\left[Q_{m, 0}, \mathcal{U}_{m}(t, s)\right]=0
$$

As a consequence if by definition

$$
\mathcal{U}_{\text {eff }, m}(t, s):=Q_{m, 0} \mathcal{U}_{m}(t, s) Q_{m, 0}
$$

then $\mathcal{U}_{\text {eff }, m}(t, s)$ satisfies (as operators in $Q_{m, 0} \mathcal{H}$ ) the equation of motion:

$$
\begin{aligned}
i \frac{d \mathcal{U}_{\mathrm{eff}, m}(t, s)}{d t} & =Q_{m, 0}\left(i \dot{W}_{m}(t)^{*} W_{m}(t)+W_{m}(t)^{*} H(t) W_{m}(t)\right) Q_{m, 0} \mathcal{U}_{\mathrm{eff}, m}(t, s) \\
& =H_{\mathrm{eff}, m}(t) \mathcal{U}_{\mathrm{eff}, m}(t, s) .
\end{aligned}
$$

Going backwards, once $\mathcal{U}_{\text {eff }, m}(t, s)$ is known one can recover $U_{m}^{A}(t, s)$ when restricted to $Q_{m}\left(t_{0}\right) \mathcal{H}$ :

$$
U_{m}^{A}\left(t, t_{0}\right) Q_{m}\left(t_{0}\right)=W_{m}(t) \mathcal{U}_{\mathrm{eff}, m}\left(t, t_{0}\right) W_{m}\left(t_{0}\right)^{*} Q_{m}\left(t_{0}\right) .
$$

The point of the reduction theory outlined above is that we can compute $H_{\mathrm{eff}, m}(t)$ up to a controlled error:

Theorem 4.1: For sufficiently large $m$

$$
\begin{aligned}
H_{\mathrm{eff}, m}(t) & =Q_{m, 0}\left(H_{0}(t)+V\right) Q_{m, 0} \\
& +\frac{1}{2} Q_{m, 0}\left(\hat{E}_{m, 1}(t) V+V \hat{E}_{m, 1}(t)\right) Q_{m, 0}+\Delta H_{\mathrm{eff}, m}(t)
\end{aligned}
$$

where

$$
\hat{E}_{m, 1}(t):=\frac{1}{2 \pi i} \oint_{\gamma_{m}} d z R_{0}(z, t) V R_{0}(z, t)
$$

and

$$
\sup _{t \in I_{0}}\left\|\Delta H_{\mathrm{eff}, m}(t)\right\| \leq C_{V}\langle m\rangle b(m)^{3} .
$$

We prove the theorem in few lemmae, each lemma giving an estimate for different terms that appear in $H_{\text {eff }, m}(t)$. In the proofs we shall use several results of the perturbation theory. As well known, $Q_{m}(t)$ has the following expansion

$$
Q_{m}(t)=\sum_{j=0}^{N} \hat{E}_{m, j}(t)+E_{m, N+1}(t)
$$

with

$$
\begin{aligned}
\hat{E}_{m, j}(t) & :=\frac{(-1)^{j} i}{2 \pi} \oint_{\gamma_{m}} d z R_{0}(z, t)\left(V R_{0}(z, t)\right)^{j} \\
E_{m, j}(t) & :=\frac{(-1)^{j} i}{2 \pi} \oint_{\gamma_{m}} d z\left(R_{0}(z, t) V\right)^{j} R(z, t) .
\end{aligned}
$$


Using the identity $Q_{m}(t)=\left(Q_{m}(t)\right)^{2}$ one can easily check the following relations

$$
\begin{aligned}
Q_{m, 0} \hat{E}_{m, 1}(t) Q_{m, 0} & =0 \\
Q_{m, 0} \hat{E}_{m, 2}(t) Q_{m, 0} & =-Q_{m, 0} \hat{E}_{m, 1}^{2}(t) Q_{m, 0} \\
{\left[H_{0}(t), \hat{E}_{m, 1}(t)\right] } & =-\left[V, Q_{m, 0}\right] .
\end{aligned}
$$

Moreover, $E_{m, j}(t)$ and its derivatives obey the estimates

$$
\begin{aligned}
& \sup _{t \in I_{0}}\left\|E_{m, j}(t)\right\| \leq C_{V}(b(m))^{j}, \\
& \sup _{t \in I_{0}}\left\|\dot{E}_{m, j}(t)\right\| \leq C_{V}(j+1)(b(m))^{j} .
\end{aligned}
$$

To see (4.21) we deform the contour $\gamma_{m}$ into $\Gamma_{m-1} \cup \Gamma_{m}$ and write

$$
\begin{aligned}
\sup _{t \in I_{0}}\left\|E_{m, j}(t)\right\| & =\frac{1}{2 \pi} \sup _{t \in I_{0}}\left\|\int_{\Gamma_{m-1} \cup \Gamma_{m}} d z\left|R_{0}(z, t)\right|^{\frac{1}{2}}(K(z, t))^{j}(1+K(z, t))^{-1} R_{0}(z, t)^{\frac{1}{2}}\right\| \\
& \leq \frac{2}{2 \pi} \sup _{t \in I_{0}} \sup _{z \in \Gamma_{m-1} \cup \Gamma_{m}}\left\|(K(z, t))^{j-1}\right\| \int_{\Gamma_{m-1} \cup \Gamma_{m}}|d z|\left\|R_{0}(z, t)^{\frac{1}{2}}\right\|^{2}\|K(z, t)\| \\
& \leq C_{V}(b(m))^{j},
\end{aligned}
$$

where in the last line we used Lemma 2.3. The estimate (4.22) is obtained in the same way. We remark without giving details that $\hat{E}_{m, j}$ and $\dot{\hat{E}}_{m, j}$ verify the same estimates as $E_{m, j}$ and $\dot{E}_{m, j}$. Now we list the estimates for the various term appearing in $H_{\mathrm{eff}, m}$ :

Lemma 4.2: The first term in the effective Hamiltonian obeys the following estimate:

$$
\sup _{t \in I_{0}}\left\|Q_{m, 0} \dot{W}_{m}(t)^{*} W_{m}(t) Q_{m, 0}\right\| \leq C_{V}\langle m\rangle b(m)^{3}
$$

Lemma 4.3: The second term is estimated as follows

$$
\begin{aligned}
Q_{0} W_{m}^{*}(t) H_{0}(t) W_{m}(t) Q_{0} & =Q_{0} H_{0}(t) Q_{0}-\frac{1}{2}\left\{Q_{0} V \hat{E}_{m, 1}(t) Q_{0}+Q_{0} \hat{E}_{m, 1}(t) V Q_{0}\right\} \\
& +\mathcal{O}_{r}\left(\langle m\rangle b(m)^{3}\right)
\end{aligned}
$$

Lemma 4.4: The last term gives

$$
Q_{0} W(t)_{m}^{*} V W_{m}(t) Q_{0}=Q_{0} V Q_{0}+Q_{0}\left(V \hat{E}_{m, 1}(t)+\hat{E}_{m, 1}(t) V\right) Q_{0}+\mathcal{O}_{r}\left(\langle m\rangle(b(m))^{3}\right)
$$

Combining now Lemmae 4.2, 4.3 and 4.4 one obtains the explicit form of the effective Hamiltonian as given in Theorem 4.1. When proving the lemmae we skip 
for simplicity the index $m$ and the time dependence of $W_{m}(t), E_{m, 1}(t), Q_{m}(t)$ and other related quantities.

Proof of Lemma 4.2: We shall use the fact that $E_{1}, \hat{E}_{1}$ and $E_{2}$ are symmetric hence in particular $\partial_{t}\left(1-E_{1}^{2}\right)^{-\frac{1}{2}}$ is also symmetric. As we have said it is easy to see that

$$
E_{1}=\mathcal{O}_{r}(b(m)), \quad \hat{E}_{1}=\mathcal{O}_{r}(b(m)) \quad E_{2}=\mathcal{O}_{r}\left(b^{2}(m)\right) .
$$

Denoting $L:=\left(1-E_{1}^{2}\right)^{-\frac{1}{2}}=: 1+M:=1+E_{1}^{2} F$ one has:

$$
W Q_{0}=L Q Q_{0}, \quad Q_{0} \dot{W}^{*}=Q_{0} \dot{Q} L+Q_{0} Q \dot{L}
$$

and

$$
M=\mathcal{O}_{r}\left(b^{2}(m)\right)
$$

Actually by writing $L^{2}=1+\tilde{N}$ we obtain

$$
\begin{aligned}
Q_{0} \dot{W}^{*} W Q_{0} & =\frac{1}{2} Q_{0}\left(\dot{W}^{*} W-W^{*} \dot{W}\right) Q_{0} \\
& =\frac{1}{2} Q_{0}\left(\dot{Q} L^{2} Q-Q L^{2} \dot{Q}\right) Q_{0}+\frac{1}{2} Q_{0} Q[\dot{L}, L] Q Q_{0} \\
& =\frac{1}{2} Q_{0}[\dot{Q}, Q] Q_{0}+\frac{1}{2} Q_{0}(\dot{Q} \tilde{N} Q-Q \tilde{N} \dot{Q}) Q_{0}+\frac{1}{2} Q_{0} Q[\dot{\tilde{N}}, \tilde{N}] Q Q_{0} \\
& =\frac{1}{2} Q_{0}[\dot{Q}, Q] Q_{0}+\mathcal{O}_{r}\left(b^{3}(m)\right)
\end{aligned}
$$

where we used that $\tilde{N}$ and $\dot{\tilde{N}}$ are of $\mathcal{O}_{r}\left(b^{2}(m)\right)$ and $\dot{Q}=\mathcal{O}_{r}(b(m))$. Now one should use the expansion $Q=Q_{0}+\hat{E}_{1}+E_{2}$ and the property $Q_{0} \hat{E}_{1} Q_{0}=0$

$$
\begin{aligned}
Q_{0}[\dot{Q}, Q] Q_{0} & =Q_{0}\left[\dot{\hat{E}}_{1}+\dot{E}_{2}, Q_{0}+\hat{E}_{1}+E_{2}\right] Q_{0} \\
& =Q_{0}\left[\hat{\hat{E}}_{1}, \hat{E}_{1}\right] Q_{0}+\mathcal{O}_{r}\left(b^{3}(m)\right) .
\end{aligned}
$$

The last thing to be done is to show that $Q_{0}\left[\dot{\hat{E}}_{1}, \hat{E}_{1}\right] Q_{0}=0$ This follows by writing explicitely $\hat{E}_{1}$ (that is, by using residue theorem) and by direct calculation.

Proof of Lemma 4.3: The idea behind the proof is to write

$$
Q_{0} W^{*} H_{0} W Q_{0}=Q_{0} W^{*} \hat{H}_{0} W Q_{0}+(m-1)^{2} Q_{0}
$$

with $\hat{H}_{0}:=H_{0}-(m-1)^{2} Q_{0}$ and to estimate the first term. As $W=\left(1+E_{1}^{2} F\right)\left(Q Q_{0}+\right.$ $\left.(1-Q)\left(1-Q_{0}\right)\right)$ then

$$
W Q_{0}=Q Q_{0}+E_{1}^{2} F Q Q_{0}
$$

from where

$$
\begin{aligned}
Q_{0} W^{*} \hat{H}_{0} W Q_{0} & =Q_{0} Q\left(1+F E_{1}^{2}\right) \hat{H}_{0}\left(1+E_{1}^{2} F\right) Q Q_{0} \\
& =Q_{0} Q \hat{H}_{0} Q Q_{0}+Q_{0} Q\left(\hat{H}_{0} E_{1}^{2} F+h . c\right)+Q_{0} Q F E_{1}^{2} \hat{H}_{0} E_{1}^{2} F Q Q_{0}
\end{aligned}
$$


We start by estimating the last term

$$
\sup _{t \in I_{0}}\left\|F E_{1}^{2} \hat{H}_{0} E_{1}^{2} F\right\| \leq c t .\left\|E_{1}^{2}\right\| \cdot\left\|E_{1} \hat{H}_{0} E_{1}\right\|
$$

which requires an estimate on $E_{1} \hat{H}_{0} E_{1}$. To obtain it we shall use

$$
\sup _{t \in I_{0}} \sup _{z, z^{\prime} \in \Gamma_{m-1} \cup \Gamma_{m}}\left\|R_{0}\left(z^{\prime}, t\right)^{\frac{1}{2}} \hat{H}_{0} R_{0}(z, t)^{\frac{1}{2}}\right\| \leq C .
$$

It follows from this that

$$
\begin{aligned}
& \sup _{t \in I_{0}}\left\|E_{1} \hat{H}_{0} E_{1}\right\| \\
= & \sup _{t \in I_{0}}\left\|\frac{1}{2 \pi^{2}} \oint_{\gamma_{m}} \oint_{\gamma_{m}} d z d z^{\prime} R(z, t) V R_{0}(z, t) \hat{H}_{0} R_{0}\left(z^{\prime}, t\right) V R\left(z^{\prime}, t\right)\right\| \\
= & \sup _{t \in I_{0}} \| \frac{1}{2 \pi^{2}} \oint_{\gamma_{m}} \oint_{\gamma_{m}} d z d z^{\prime} R_{0}(z, t)^{\frac{1}{2}}(1+K(z, t))^{-1} K(z, t)\left|R_{0}(z, t)\right|^{\frac{1}{2}} . \\
\cdot & \hat{H}_{0}\left|R_{0}\left(z^{\prime}, t\right)\right|^{\frac{1}{2}} K\left(z^{\prime}, t\right)\left(1+K\left(z^{\prime}, t\right)\right)^{-1}\left|R_{0}\left(z^{\prime}, t\right)\right|^{\frac{1}{2}} \\
\leq & C \oint_{\gamma_{m}}|d z|\left\|R_{0}(z, t)^{\frac{1}{2}}\right\|\|K(z, t)\| \cdot \oint_{\gamma_{m}}\left|d z^{\prime}\right|\left\|R_{0}\left(z^{\prime}, t\right)^{\frac{1}{2}}\right\|\left\|K\left(z^{\prime}, t\right)\right\| \\
\leq & C_{V}\langle m\rangle b(m)^{2} .
\end{aligned}
$$

Then the term $F E_{1}^{2} \hat{H}_{0} E_{1}^{2} F$ turns out to be of order $\mathcal{O}_{r}\left(\langle m\rangle b(m)^{4}\right)$ and can be disregarded. We continue the estimations with $Q_{0} Q F E_{1}^{2} \hat{H}_{0} Q Q_{0}$ using the perturbation theory up to $E_{1}$ (i.e $Q=Q_{0}+E_{1}$ ):

$$
\begin{aligned}
Q_{0} Q F E_{1}^{2} \hat{H}_{0} Q Q_{0} & =Q_{0} F E_{1}^{2} \hat{H}_{0} Q_{0}+Q_{0} F E_{1}^{2} \hat{H}_{0} E_{1} Q_{0} \\
& +Q_{0} E_{1} F E_{1}^{2} \hat{H}_{0} E_{1} Q_{0}+Q_{0} E_{1} F E_{1}^{2} \hat{H}_{0} Q_{0} .
\end{aligned}
$$

The second and the third terms in the above equation are simply estimated using the bounds on $E_{1}$ and $E_{1} \hat{H}_{0} E_{1}$. For the fourth term we have to estimate instead $Q_{0} \hat{H}_{0} E_{1}$. Following the same steps as in the estimation of $E_{1} \hat{H}_{0} E_{1}$ we arrive at

$$
\begin{aligned}
\sup _{t \in I_{0}}\left\|Q_{0} \hat{H}_{0} E_{1}(t)\right\| & \left.=\sup _{t \in I_{0}} \| \frac{1}{2 \pi^{2}} \oint_{\gamma_{m}} \oint_{\gamma_{m}} d z d z^{\prime} R_{0}(z, t) \hat{H}_{0}(t) R_{0}\left(z^{\prime}, t\right) V\left(z^{\prime}, t\right)\right) R\left(z^{\prime}, t\right) \| \\
& \leq C_{V}\langle m\rangle b(m)
\end{aligned}
$$

In the similar way one can prove a more general result that will be used below, namely

$$
\sup _{t \in I_{0}}\left\|Q_{0} \hat{H}_{0} E_{j}\right\| \leq C_{V}\langle m\rangle(b(m))^{j}
$$

It follows then from (4.30) that

$$
\sup _{t \in I_{0}}\left\|Q_{0} E_{1} F E_{1}^{2} \hat{H}_{0} Q_{0}\right\| \leq C\left\|E_{1}\right\|^{2}\left\|E_{1} \hat{H}_{0} Q_{0}\right\| \leq C_{V}\langle m\rangle(b(m))^{3} .
$$


Hence a preliminar result is

$$
\begin{aligned}
Q_{0} W^{*} \hat{H}_{0} W Q_{0} & =Q_{0} Q \hat{H}_{0} Q Q_{0}+Q_{0} Q\left(F E_{1}^{2} \hat{H}_{0}+\hat{H}_{0} E_{1}^{2} F\right) Q Q_{0} \\
& +\mathcal{O}_{r}\left(\langle m\rangle(b(m))^{3}\right) .
\end{aligned}
$$

To go further we shall use that $F=\frac{1}{2}+E_{1}^{2} G$ ( $G$ and its derivatives being again uniformely bounded). Then

$$
Q_{0} Q F E_{1}^{2} \hat{H}_{0} Q Q_{0}=\frac{1}{2} Q_{0} Q E_{1}^{2} \hat{H}_{0} Q Q_{0}+Q_{0} Q E_{1}^{2} G E_{1}^{2} \hat{H}_{0} Q Q_{0}
$$

and the last term is of order $\mathcal{O}_{r}\left(\langle m\rangle(b(m))^{4}\right)$. In what concerns $Q_{0} Q E_{1}^{2} \hat{H}_{0} Q Q_{0}$ one should use the perturbation theory up to $E_{1}$ :

$$
Q_{0} Q E_{1}^{2} \hat{H}_{0} Q Q_{0}=Q_{0} E_{1}^{2} \hat{H}_{0} Q_{0}+\mathcal{O}_{r}\left(\langle m\rangle(b(m))^{3}\right)
$$

so finally

$$
\begin{aligned}
& Q_{0} W^{*} \hat{H}_{0} W Q_{0}=Q_{0} \hat{H}_{0} Q_{0}+Q_{0}\left(\hat{H}_{0} E_{1}+E_{1} \hat{H}_{0}\right) Q_{0} \\
& +\frac{1}{2} Q_{0}\left(\hat{H}_{0} E_{1}^{2}+E_{1}^{2} \hat{H}_{0}\right) Q_{0}+Q_{0} E_{1} \hat{H}_{0} E_{1} Q_{0}+\mathcal{O}_{r}\left(\langle m\rangle(b(m))^{3}\right) .
\end{aligned}
$$

In the following we shall use the expansion $E_{1}=\hat{E}_{1}+\ldots+\hat{E}_{N}+E_{N+1}$ for suitable $N$ and the estimate (4.31), in such a way that the terms containing $E_{N+1}$ are small. It turns out that in order to estimate the third and the fourth terms it is sufficient to go up to $E_{2}$. When plugging this expansion in $Q_{0} \hat{H}_{0} E_{1}^{2} Q_{0}$ and $Q_{0} E_{1} \hat{H}_{0} E_{1} Q_{0}$ several new terms will appear $\left(Q_{0} \hat{H}_{0} \hat{E}_{1} E_{2} Q_{0}, Q_{0} \hat{E}_{1} \hat{H}_{0} E_{2}\right.$ and $\left.Q_{0} E_{2} \hat{H}_{0} E_{2}\right)$. All of them are easily estimated in the same way as before and the result is:

$$
\begin{aligned}
Q_{0} \hat{H}_{0} E_{1}^{2} Q_{0} & =Q_{0} \hat{H}_{0} \hat{E}_{1}^{2} Q_{0}+\mathcal{O}_{r}\left(\langle m\rangle(b(m))^{3}\right) \\
Q_{0} E_{1} \hat{H}_{0} E_{1} Q_{0} & =Q_{0} \hat{E}_{1} \hat{H}_{0} \hat{E}_{1}+\mathcal{O}_{r}\left(\langle m\rangle(b(m))^{3}\right) .
\end{aligned}
$$

For the first term we have to go up to $E_{3}$ with the expansion. Using (4.31) and the identity (4.18) we arrive at

$$
Q_{0} \hat{H}_{0} E_{1} Q_{0}=Q_{0} \hat{H}_{0} \hat{E}_{2} Q_{0}+\mathcal{O}_{r}\left(\langle m\rangle(b(m))^{3}\right) .
$$

Moreover, using (4.19) we can replace $Q_{0} \hat{E}_{2} Q_{0}$ and write

$$
\begin{aligned}
& Q_{0} W^{*} H_{0} W Q_{0}=Q_{0} \hat{H}_{0}+Q_{0}\left(n_{0}-1\right)^{2}-\frac{1}{2} Q_{0}\left(\hat{H}_{0} \hat{E}_{1}^{2}+\hat{E}_{1}^{2} \hat{H}_{0}\right) Q_{0} \\
& +\frac{1}{2}\left(Q_{0} \hat{E}_{1} \hat{H}_{0} \hat{E}_{1} Q_{0}+Q_{0} \hat{E}_{1} \hat{H}_{0} \hat{E}_{1} Q_{0}\right)+\mathcal{O}_{r}\left(\langle m\rangle(b(m))^{3}\right) \\
& =Q_{0} H_{0}+\frac{1}{2}\left(Q_{0}\left[\hat{E}_{1}, \hat{H}_{0}\right] \hat{E}_{1} Q_{0}+Q_{0} \hat{E}_{1}\left[\hat{H}_{0}, \hat{E}_{1}\right]\right)+\mathcal{O}_{r}\left(\langle m\rangle(b(m))^{3}\right) \\
& =Q_{0} H_{0} Q_{0}-\frac{1}{2}\left\{Q_{0} V \hat{E}_{1} Q_{0}+Q_{0} \hat{E}_{1} V Q_{0}\right\} \\
& +\mathcal{O}_{r}\left(\langle m\rangle(b(m))^{3}\right) .
\end{aligned}
$$


In the last line we used (4.20). The lemma is now proved

Proof of Lemma 4.4: Using (4.27) and $Q=Q_{0}+E_{1}$ it results

$$
\begin{aligned}
Q_{0} W(t)^{*} V W(t) Q_{0} & =Q_{0} V Q_{0}+Q_{0}\left(E_{1} V+V E_{1}\right) Q_{0}+Q_{0} E_{1} V E_{1} Q_{0} \\
& +Q_{0}\left(Q_{0}+E_{1}\right)\left(V E_{1}^{2} F+F E_{1}^{2} V\right)\left(Q_{0}+E_{1}\right) Q_{0} \\
& +Q_{0}\left(Q_{0}+E_{1}\right) F E_{1}\left(E_{1} V E_{1}\right) E_{1} F\left(Q_{0}+E_{1}\right) Q_{0}
\end{aligned}
$$

Let us first show that the last three terms are small. To see this we need estimates on $E_{1} V E_{1}$ and $Q_{0} V E_{1}$. For $E_{1} V E_{1}$ one has

$$
\begin{array}{r}
E_{1} V E_{1}=-\frac{1}{2 \pi^{2}} \oint_{\gamma_{m}} \oint_{\gamma_{m}} d z d z^{\prime} R_{0}(z, t)^{\frac{1}{2}} K(z, t)(1+K(z, t))^{-1} \\
\cdot\left|R_{0}(z, t)\right|^{\frac{1}{2}} V R_{0}\left(z^{\prime}, t\right)^{\frac{1}{2}} K\left(z^{\prime}, t\right)\left(1+K\left(z^{\prime}, t\right)\right)^{-1}\left|R_{0}\left(z^{\prime}, t\right)\right|^{\frac{1}{2}}
\end{array}
$$

Now observe that

$$
\sup _{t \in I_{0}} \sup _{z \in \gamma_{m}} \sup _{z^{\prime} \in \gamma_{m}}\left\|\left|R_{0}(z, t)\right|^{\frac{1}{2}} V R_{0}\left(z^{\prime}, t\right)^{\frac{1}{2}}\right\| \leq C_{V} b(m) .
$$

Then using the fact that $l\left(\gamma_{m}\right) \sim\langle m\rangle$

$$
\sup _{t \in I_{0}}\left\|E_{1} V E_{1}\right\| \leq C_{V}\langle m\rangle(b(m))^{3} .
$$

In what concerns $Q_{0} V E_{1}$ we have

$$
\begin{aligned}
\sup _{t \in I_{0}}\left\|Q_{0} V E_{1}\right\| & =\frac{1}{2 \pi}\left\|\oint_{\gamma_{m}} \oint_{\gamma_{m}} d z d z^{\prime} R_{0}(z, t) V R_{0}\left(z^{\prime}, t\right) V R\left(z^{\prime}, t\right)\right\| \\
& \leq C_{V}\langle m\rangle(b(m))^{2}
\end{aligned}
$$

The estimates (4.39) and (4.40) used in $Q_{0} W(t)^{*} V W(t) Q_{0}$ suffices to prove that the last three terms are of order $\mathcal{O}_{r}\left(\langle m\rangle(b(m))^{3}\right)$. We arrived thus at

$$
\begin{aligned}
Q_{0} W^{*} V W Q_{0} & =Q_{0} V Q_{0}+Q_{0}\left(V E_{1}+E_{1} V\right) Q_{0} \\
& +\mathcal{O}_{r}\left(\langle m\rangle(b(m))^{3}\right)
\end{aligned}
$$

As for the remaining term $Q_{0} E_{1} V Q_{0}$ we shall write it more carefully by writing

$$
Q_{0} E_{1} V Q_{0}=Q_{0}\left(\hat{E}_{1}+E_{2}\right) V Q_{0}
$$

Finally, $Q_{0} E_{2} V Q_{0}$ is found to be of order $\mathcal{O}_{r}\left(\langle m\rangle(b(m))^{3}\right)$ and the lemma is finished.

For $\tilde{H}_{\text {eff }, m}$ the computations and estimations are similar as above the difference appearing again due to the different integration contour. We can then conclude that

$\tilde{H}_{\mathrm{eff}, m}(t)=\tilde{Q}_{m, 0}\left(H_{0}(t)+V\right) \tilde{Q}_{m, 0}+\frac{1}{2} \tilde{Q}_{m, 0}\left(\hat{E}_{m, 1}(t) V+V \hat{E}_{m, 1}(t)\right) \tilde{Q}_{m, 0}+\Delta \tilde{H}_{\mathrm{eff}, m}(t)$

where $\hat{E}_{m, 1}(t)$ is now the first order term from the pertubative expansion for $\tilde{Q}_{m}(t)$ and the remainder $\Delta \tilde{H}_{\text {eff }, m}(t)$ is of the same order as in Theorem 4.2. 


\section{The adiabatic evolution. The transition ampli- tudes}

We shall use now the effective Hamiltonian obtained in the previous section to compute $U_{m}^{A}\left(t_{1}, t_{0}\right)$ and $\tilde{U}_{m}^{A}\left(t_{2}, t_{1}\right)$ when restricted to $Q_{m}\left(t_{0}\right)$ and $\tilde{Q}_{m}\left(t_{1}\right)$ respectively, up to an error which remains small after taking the sum over $m$ from some sufficiently large $n_{0}$ to infinity. Remember that $t_{0}=-\frac{1}{4}, t_{1}=\frac{1}{4}, t_{2}=\frac{3}{4}$ and that $\left\{\varphi_{\alpha}\left(t_{l}\right)\right\}_{\alpha}$, $l=0,1,2$ are the canonical eigenbasis for $H(t)$ (see (2.50) ). The main result of this section is contained in:

Theorem 5.1 Let $r>-\frac{1}{2}, m$ sufficiently large and define the transfer matrices

$$
\begin{aligned}
& \mathcal{S}^{m}\left(t_{1}, t_{0}\right)=\left(\begin{array}{cc}
\mathcal{S}_{2 m-1,2 m-2}^{m}\left(t_{1}, t_{0}\right) & \mathcal{S}_{2 m-1,2 m-1}^{m}\left(t_{1}, t_{0}\right) \\
\mathcal{S}_{2 m-2,2 m-2}^{m}\left(t_{1}, t_{0}\right) & \mathcal{S}_{2 m-2,2 m-1}^{m}\left(t_{1}, t_{0}\right)
\end{array}\right) \\
& \tilde{\mathcal{S}}^{m}\left(t_{2}, t_{1}\right)=\left(\begin{array}{cc}
\tilde{\mathcal{S}}_{2 m, 2 m-1}^{m}\left(t_{2}, t_{1}\right) & \tilde{\mathcal{S}}_{2 m, 2 m}^{m}\left(t_{2}, t_{1}\right) \\
\tilde{\mathcal{S}}_{2 m-1,2 m-1}^{m}\left(t_{2}, t_{1}\right) & \tilde{\mathcal{S}}_{2 m-1,2 m}^{m}\left(t_{2}, t_{1}\right)
\end{array}\right)
\end{aligned}
$$

with

$$
\begin{array}{lll}
\mathcal{S}_{i, j}^{m}\left(t_{1}, t_{0}\right):=\left\langle\varphi_{i}\left(t_{1}\right), U_{m}^{A}\left(t_{1}, t_{0}\right) \varphi_{j}\left(t_{0}\right)\right\rangle, & i, j=2 m-2,2 m-1 \\
\tilde{\mathcal{S}}_{i, j}^{m}\left(t_{2}, t_{1}\right):=\left\langle\varphi_{i}\left(t_{2}\right), \tilde{U}_{m}^{A}\left(t_{2}, t_{1}\right) \varphi_{j}\left(t_{1}\right)\right\rangle, & i, j=2 m-1,2 m .
\end{array}
$$

Then

$$
\begin{aligned}
\mathcal{S}^{m}\left(t_{1}, t_{0}\right) & =e^{-i \theta_{m}\left(t_{1}, t_{0}\right)}\left(\begin{array}{cc}
\alpha_{2 m-2}\left(t_{1}, t_{0}\right) & -\bar{\beta}_{2 m-2}\left(t_{1}, t_{0}\right) \\
\beta_{2 m-2}\left(t_{1}, t_{0}\right) & \alpha_{2 m-2}^{\prime}\left(t_{1}, t_{0}\right)
\end{array}\right)+\mathcal{R}_{\mathrm{eff}, m}, \\
\tilde{\mathcal{S}}^{m}\left(t_{2}, t_{1}\right) & =e^{-i \tilde{\theta}_{m}\left(t_{2}, t_{1}\right)}\left(\begin{array}{cc}
\tilde{\alpha}_{2 m-1}\left(t_{2}, t_{1}\right) & -\tilde{\tilde{\beta}}_{2 m-1}\left(t_{2}, t_{1}\right) \\
\tilde{\beta}_{2 m-1}\left(t_{2}, t_{1}\right) & \tilde{\alpha}_{2 m-1}^{\prime}\left(t_{2}, t_{1}\right)
\end{array}\right)+\mathcal{R}_{\mathrm{eff}, m},
\end{aligned}
$$

with:

$$
\mathcal{R}_{\text {eff }, m}=\mathcal{O}_{r}\left(\frac{\log ^{8}\langle m\rangle}{\langle m\rangle^{\min \left\{2+8 r, \frac{3}{2}+5 r, \frac{3}{2}+r, 2\right\}}}\right) \quad \text { as } m \rightarrow \infty
$$

and the following notations (we list below only the quantities related to $\mathcal{S}^{m}$; the ones corresponding to $\tilde{\mathcal{S}}^{m}$ are discussed at the end of this section)

$$
\begin{aligned}
\alpha_{2 m-2}\left(t_{1}, t_{0}\right) & :=1-\frac{|\hat{V}(2(m-1))|^{2}}{m-1} c_{2, m}\left(t_{1}, t_{0}\right)-i \omega_{1, m}\left(t_{1}, t_{0}\right) \\
\alpha_{2 m-2}^{\prime}\left(t_{1}, t_{0}\right) & :=1-\frac{|\hat{V}(2(m-1))|^{2}}{m-1} c_{2, m}\left(t_{1}, t_{0}\right)-i \omega_{2, m}\left(t_{1}, t_{0}\right) \\
\beta_{2 m-2}\left(t_{1}, t_{0}\right) & :=\frac{\hat{V}(2(m-1))}{\sqrt{m-1}} c_{1, m}\left(t_{1}, t_{0}\right)-i \lambda_{m}\left(t_{1}, t_{0}\right)-\frac{2 \hat{V}(2(m-1))}{m-1},(5 \\
c_{1, m}\left(t_{1}, t_{0}\right) & :=-(i+1) \frac{\sqrt{\pi}}{2} e^{i \frac{m-1}{8}} \operatorname{erf}\left(\frac{i+1}{4} \sqrt{m-1}\right) \\
c_{2, m}\left(t_{1}, t_{0}\right) & :=\frac{\pi}{4}\left|\operatorname{erf}\left(\frac{i-1}{4} \sqrt{m-1}\right)\right|^{2}
\end{aligned}
$$




$$
\begin{aligned}
& \theta_{m}\left(t_{1}, t_{0}\right):=\int_{t_{0}}^{t_{1}} d s\left((m-1)^{2}+s^{2}\right), \\
& \lambda_{m}\left(t_{1}, t_{0}\right):=\int_{t_{0}}^{t_{1}} d s \bar{\Delta}_{m}(s) e^{-2 i(m-1)\left(s^{2}-t_{0}^{2}\right)}, \\
& \omega_{1, m}\left(t_{1}, t_{0}\right):=\int_{t_{0}}^{t_{1}} d s \frac{1}{2}\left\langle\varphi_{m-1,0},\left(V \hat{E}_{1}(t)+\hat{E}_{1}(t) V\right) \varphi_{m-1,0}\right\rangle \\
&=: \int_{t_{0}}^{t_{1}} d s \gamma_{1, m}(s) \\
& \omega_{2, m}\left(t_{1}, t_{0}\right):=\int_{t_{0}}^{t_{1}} d s \frac{1}{2}\left\langle\varphi-(m-1), 0,\left(V \hat{E}_{1}(t)+\hat{E}_{1}(t) V\right) \varphi-(m-1), 0\right\rangle \\
&=: \int_{t_{0}}^{t_{1}} d s \gamma_{2, m}(s), \\
& \Delta_{m}(t):=\frac{1}{2}\left\langle\varphi_{m-1,0},\left(V \hat{E}_{1}(t)+\hat{E}_{1}(t) V\right) \varphi_{-(m-1), 0}\right\rangle .
\end{aligned}
$$

Here $\operatorname{erf}(z)$ is the error function (see [1]):

$$
\operatorname{erf}(z):=\frac{2}{\sqrt{\pi}} \int_{0}^{z} e^{-t^{2}} d t
$$

Remark: Although the above estimates are valid for $r>-\frac{1}{2}$ the bound on the error terms gets small as $m$ tends to infinity only for $r>-\frac{1}{4}$ and is summable with respect to $m$ only for $r>-\frac{1}{10}$ (these facts can be noticed from the behaviour of the error term $\left.\mathcal{R}_{\mathrm{eff}, m}\right)$.

Proof: We shall consider only $S_{i, j}^{m}$; the proof for $\tilde{S}_{i, j}^{m}$ is similar. From (2.50) and (4.11):

$$
S_{i, j}^{m}\left(t_{1}, t_{0}\right)=\sum_{k, l=2 m-2,2 m-1} \bar{c}_{k, i}^{m}\left(t_{1}\right) \mathcal{U}_{\mathrm{eff}, m ; k, l}\left(t_{1}, t_{0}\right) c_{l, j}^{m}\left(t_{0}\right)
$$

where $\varphi_{\alpha}^{0}\left(t_{\beta}\right)=\varphi_{n_{\alpha}, 0}\left(t_{\beta}\right)$ (see (2.32) $)$ ) and we introduced the coefficients

$$
c_{i, k}^{m}\left(t_{p}\right):=\left\langle\varphi_{i}^{0}\left(t_{p}\right), W_{m}\left(t_{p}\right)^{*} N_{k}\left(t_{p}\right) \varphi_{k}^{0}\left(t_{p}\right)\right\rangle, \quad p=0,1
$$

and

$$
\mathcal{U}_{\text {eff }, m ; k, l}\left(t_{1}, t_{0}\right):=\left\langle\varphi_{k}^{0}\left(t_{1}\right), \mathcal{U}_{\text {eff }, m}\left(t_{1}, t_{0}\right) \varphi_{l}^{0}\left(t_{0}\right)\right\rangle .
$$

The estimation of the coefficients $c_{i, k}^{m}\left(t_{p}\right)$ is easy and reads:

Lemma 5.2: For $m$ large enough and $r>-\frac{1}{2}$ one has:

$$
c_{\alpha, \beta}^{m}\left(t_{p}\right)=\delta_{\alpha, \beta}+\frac{\left\langle\varphi_{\alpha}^{0}\left(t_{p}\right), V \varphi_{\beta}^{0}\left(t_{p}\right)\right\rangle}{E_{\beta}^{0}\left(t_{p}\right)-E_{\alpha}^{0}\left(t_{p}\right)}\left(1-\delta_{\alpha, \beta}\right)+\mathcal{O}_{r}\left(b^{2}(m)\right), \quad \text { as } m \rightarrow \infty .
$$


The estimation of $\mathcal{U}_{\text {eff, } m}$ is more involved and the result is summarised in:

Lemma 5.3: For $m$ large enough and $r>-\frac{1}{2}$ the effective evolution is given by:

$$
\mathcal{U}_{\mathrm{eff}, m ; i, j}\left(t_{1}, t_{0}\right)=\mathcal{S}_{i j}^{m}\left(t_{1}, t_{0}\right)+e^{-i \theta_{m}\left(t_{1}, t_{0}\right)} v_{m}+\mathcal{R}_{\mathrm{eff}, m}, \quad \text { as } m \rightarrow \infty .
$$

with

$$
v_{m}:=\left(\begin{array}{cc}
0 & -\frac{2 \hat{V}(2(m-1))}{m-1} \\
\frac{2 \hat{V}(2(m-1))}{m-1} & 0
\end{array}\right) .
$$

The proof of Thm. 5.1 requires only straightforward calculations using (5.18), (5.21) and (5.22).

Proof of Lemma 5.2: Write (see (4.16) and (4.17))

$$
Q_{m}\left(t_{p}\right)-Q_{m}^{0}\left(t_{p}\right)=\hat{E}_{m, 1}\left(t_{p}\right)+E_{m, 2}\left(t_{p}\right),
$$

and in the same way

$$
\begin{aligned}
P_{\alpha}\left(t_{p}\right)-P_{\alpha}^{0}\left(t_{p}\right) & =-\frac{i}{2 \pi} \int_{C_{\alpha}} d z R_{0}\left(z, t_{p}\right) V R_{0}\left(z, t_{p}\right) \\
& +\frac{i}{2 \pi} \int_{C_{\alpha}} d z R_{0}\left(z, t_{p}\right) V R\left(z, t_{p}\right) V R_{0}\left(z, t_{p}\right) \\
& =: \quad \hat{e}_{\alpha, 1}\left(t_{p}\right)+e_{\alpha, 2}\left(t_{p}\right), \quad p=0,1
\end{aligned}
$$

with $C_{2 m-2}=\Gamma_{m-1} \cup \tilde{\Gamma}_{m-1}$ and $C_{2 m-1}=\tilde{\Gamma}_{m-1} \cup \Gamma_{m}$, i.e $C_{\alpha}$ is a countour which contains only one eigenvalue of $H_{0}\left(t_{p}\right)$ namely $E_{\alpha}^{0}\left(t_{p}\right)$. By Lemma 2.3 (see also Section 4)one has

$$
\begin{aligned}
\left\|\hat{E}_{m, 1}\left(t_{p}\right)\right\|+\left\|\hat{e}_{\alpha, 1}\left(t_{p}\right)\right\| & \leq C_{V} b(m), \\
\left\|E_{m, 2}\left(t_{p}\right)\right\|+\left\|e_{\alpha, 2}\left(t_{p}\right)\right\| & \leq C_{V} b(m)^{2} .
\end{aligned}
$$

Then using the expansion in the Sz-Nagÿ formula, (5.26), (5.27) and the fact that for $\alpha=2 m-2,2 m-1$ we have $Q_{m, 0} P_{\alpha}^{0}\left(t_{p}\right)=P_{\alpha}^{0}\left(t_{p}\right) Q_{m, 0}=P_{\alpha}^{0}\left(t_{p}\right)$ and $P_{\alpha}^{0}\left(t_{p}\right) \varphi_{\alpha}^{0}\left(t_{p}\right)=$ $\varphi_{\alpha}^{0}\left(t_{p}\right)$ it follows that up to errors of order $\mathcal{O}_{r} b(m)^{2}$

$$
c_{\alpha, \beta}^{m}\left(t_{p}\right)=\left\langle\varphi_{\alpha}^{0}\left(t_{p}\right),\left(1+\hat{E}_{m, 1}\left(t_{p}\right)+\hat{e}_{\beta, 1}\left(t_{p}\right)\right) \varphi_{\beta}^{0}\left(t_{p}\right)\right\rangle .
$$

Since $Q_{m, 0} \hat{E}_{m, 1}\left(t_{p}\right) Q_{m, 0}=P_{\beta}^{0}\left(t_{p}\right) \hat{e}_{\beta, 1}\left(t_{p}\right) P_{\beta}^{0}\left(t_{p}\right)=0$ one gets finally

$$
\left|c_{\alpha, \beta}^{m}\left(t_{p}\right)-\delta_{\alpha, \beta}-\left\langle\varphi_{\alpha}^{0}\left(t_{p}\right), \hat{e}_{\beta, 1}\left(t_{p}\right) \varphi_{\beta}^{0}\left(t_{p}\right)\left(1-\delta_{\alpha, \beta}\right)\right\rangle\right| \leq C_{V} b(m)^{2}
$$

and computing the third term in the LHS of (5.29) by residues theorem we obtain the needed result.

Proof of Lemma 5.3: First remark that due to Theorem 4.1 one can write

$$
H_{\mathrm{eff}, m}(t)=h_{\mathrm{eff}, m}(t)+\Delta H_{\mathrm{eff}, m}(t),
$$


where

$$
h_{\mathrm{eff}, m}(t)=Q_{m, 0}\left(H_{0}(t)+V\right) Q_{m, 0}+\frac{1}{2} Q_{m, 0}\left(\hat{E}_{m, 1}(t) V+V \hat{E}_{m, 1}(t)\right) Q_{m, 0} .
$$

Denote then by $\mathcal{U}_{\mathrm{eff}, m}^{0}\left(t, t_{0}\right)$ the evolution associated to $h_{\mathrm{eff}, m}(t)$ having the equation of motion

$$
i \frac{d}{d t} \mathcal{U}_{\mathrm{eff}, m}^{0}\left(t, t_{0}\right)=h_{\mathrm{eff}, m}(t) \mathcal{U}_{\mathrm{eff}, m}^{0}\left(t, t_{0}\right), \quad \mathcal{U}_{\mathrm{eff}, m}^{0}\left(t_{0}, t_{0}\right)=1
$$

The usual estimation for $\Omega_{\mathrm{eff}, m}\left(t, t_{0}\right):=\mathcal{U}_{\mathrm{eff}, m}^{0}\left(t, t_{0}\right)^{*} \mathcal{U}_{\mathrm{eff}, m}\left(t, t_{0}\right)$ gives

$$
\begin{aligned}
\sup _{t \in I_{0}}\left\|\Omega_{\mathrm{eff}, m}\left(t, t_{0}\right)-1\right\| & \leq \sup _{t \in I_{0}}\left\|i \int_{t_{0}}^{t} d s \mathcal{U}_{\mathrm{eff}, m}^{0}\left(s, t_{0}\right)^{*} \Delta H_{\mathrm{eff}, m}(s) \mathcal{U}_{\mathrm{eff}, m}^{0}\left(s, t_{0}\right) \Omega_{\mathrm{eff}, m}\left(s, t_{0}\right)\right\| \\
& \leq \frac{1}{2} \sup _{t \in I_{0}}\left\|\Delta H_{\mathrm{eff}, m}(s)\right\| \leq C_{V}\langle m\rangle b(m)^{3} .
\end{aligned}
$$

Hence

$$
\mathcal{U}_{\text {eff }, m}\left(t, t_{0}\right)=\mathcal{U}_{\mathrm{eff}, m}^{0}\left(t, t_{0}\right)+\mathcal{O}_{r}\left(\langle m\rangle b(m)^{3}\right), \quad \text { as } m \rightarrow \infty .
$$

We continue the computation of $\mathcal{U}_{\text {eff, } m}^{0}\left(t, t_{0}\right)$ by further decomposing $h_{\text {eff }, m}(t)$ into a "free" part

$$
H_{P, m}(t):=Q_{m, 0}\left(H_{0}(t)+V\right) Q_{m, 0}
$$

and a perturbation

$$
B_{m}(t):=\frac{1}{2} Q_{m, 0}\left(\hat{E}_{m, 1}(t) V+V \hat{E}_{m, 1}(t)\right) Q_{m, 0} .
$$

Remark first that $H_{P, m}(t)$ has a simple matrix form in the eigenbasis of $H_{0}(t)$ that can be explicitely written taking into account that $Q_{m, 0}=P_{m-1,0}+P_{-(m-1), 0}$. Secondly one notes that it is possible to eliminate a diagonal term from $H_{P, m}(t)$ by writing

$$
\mathcal{U}_{\mathrm{eff}, m}^{0}\left(t_{1}, t_{0}\right):=\exp \left(-i \int_{t_{0}}^{t_{1}}\left((m-1)^{2}+s^{2}\right) d s\right) \widehat{\mathcal{U}}_{\mathrm{eff}, m}\left(t_{1}, t_{0}\right),
$$

where $\widehat{\mathcal{U}}_{\text {eff }, m}\left(t, t_{0}\right)$ satisfies the equation

$$
i \frac{d}{d t} \widehat{\mathcal{U}}_{\mathrm{eff}, m}\left(t, t_{0}\right)=\left(\widehat{H}_{P, m}(t)+B_{m}(t)\right) \widehat{\mathcal{U}}_{\mathrm{eff}, m}\left(t, t_{0}\right), \quad \widehat{\mathcal{U}}_{\mathrm{eff}, m}\left(t_{0}, t_{0}\right)=1
$$

and

$$
\widehat{H}_{P, m}(t)=\left(\begin{array}{cc}
2(m-1) t & \hat{V}(2(m-1)) \\
\hat{V}(2(m-1)) & -2(m-1) t
\end{array}\right) .
$$

Now let $\widehat{\mathcal{U}}_{P, m}\left(t, t_{0}\right)$ be the evolution generated by $\widehat{H}_{P, m}(t)$ and

$$
\widehat{\Omega}_{P, m}\left(t_{1}, t_{0}\right)=\widehat{\mathcal{U}}_{P, m}\left(t_{1}, t_{0}\right)^{*} \widehat{\mathcal{U}}_{m, \text { eff }}\left(t_{1}, t_{0}\right) .
$$


We write the Dyson expansion for $\widehat{\Omega}_{P, m}$ with the remainder of order 2 :

$$
\begin{aligned}
\widehat{\Omega}_{P, m}\left(t_{1}, t_{0}\right) & =1+(-i) \int_{t_{0}}^{t_{1}} d s_{1} B_{m}^{\mathrm{int}}\left(s_{1}\right) \\
& +(-i)^{2} \int_{t_{0}}^{t_{1}} d s_{1} B_{m}^{\mathrm{int}}\left(s_{1}\right) \int_{t_{0}}^{s_{1}} d s_{2} B_{m}^{\mathrm{int}}\left(s_{2}\right) \widehat{\Omega}_{P, m}\left(s_{2}, t_{0}\right),
\end{aligned}
$$

where $B_{m}^{\text {int }}(t)=\widehat{\mathcal{U}}_{P, m}\left(t, t_{0}\right)^{*} B_{m}(t) \widehat{\mathcal{U}}_{P, m}\left(t, t_{0}\right)$. Observe first that $\sup _{t \in I_{0}} B_{m}(t)=$ $\mathcal{O}_{r}\left(\langle m\rangle b(m)^{2}\right)$ as $m \rightarrow \infty$ (this follows from the estimate on $Q_{m, 0} V \hat{E}_{1}(t)$ which is the same as the one from Eq.(4.40) ). Then

$$
\begin{aligned}
& \sup _{t \in I_{0}}\left\|\int_{t_{0}}^{t_{1}} d s_{1} B_{m}^{\mathrm{int}}\left(s_{1}\right) \int_{t_{0}}^{s_{1}} d s_{2} B_{m}^{\mathrm{int}}\left(s_{2}\right) \widehat{\Omega}_{P, m}\left(s_{2}, t_{0}\right)\right\| \leq \frac{1}{4}\left(\sup _{t \in I_{0}}\left\|B_{m}(t)\right\|\right)^{2} \\
& =\mathcal{O}_{r}\left(\left(\langle m\rangle b(m)^{2}\right)^{2}\right), \quad \text { as } m \rightarrow \infty
\end{aligned}
$$

In conclusion, at this level of approximation from (5.39) we have

$\widehat{\mathcal{U}}_{\text {eff }, m}\left(t_{1}, t_{0}\right)=\widehat{\mathcal{U}}_{P, m}\left(t_{1}, t_{0}\right)\left[1-i \int_{t_{0}}^{t_{1}} d s B_{m}^{\text {int }}(s)\right]+\mathcal{O}_{r}\left(\left(\langle m\rangle b(m)^{2}\right)^{2}\right), \quad$ as $m \rightarrow \infty$.

Thus we have reduced the problem at hand to the computation of $\widehat{\mathcal{U}}_{P, m}\left(t_{1}, t_{0}\right)$ and of the contribution of $B_{m}^{\text {int }}$. Now we write $\widehat{H}_{P, m}(t)$ in the form $\widehat{H}_{P, m}(t)=\widehat{H}_{P, m}^{0}(t)+\widehat{V}_{P}$, where

$$
\widehat{H}_{P, m}^{0}(t)=\left(\begin{array}{cc}
2(m-1) t & 0 \\
0 & -2(m-1) t
\end{array}\right), \quad \widehat{V}_{P}=\left(\begin{array}{cc}
0 & \hat{V}(2(m-1)) \\
\hat{V}(2(m-1)) & 0
\end{array}\right) .
$$

Let $\widehat{\mathcal{U}}_{P, m}^{0}$ be the evolution generated by $\widehat{H}_{P, m}^{0}$ :

$$
\widehat{\mathcal{U}}_{P, m}^{0}\left(t, t_{0}\right)=\left(\begin{array}{cc}
e^{-i(m-1)\left(t^{2}-t_{0}^{2}\right)} & 0 \\
0 & e^{i(m-1)\left(t^{2}-t_{0}^{2}\right)}
\end{array}\right) .
$$

By defining all the "effective" Hamiltonians above we ended with a time-independent perturbation $\widehat{V}_{P}$ (which is precisely the one considered in the physical literature [2, 12]). Writing the Dyson expansion with remainder of order 3 for $\widehat{\Omega}_{P, m}^{0}\left(t_{1}, t_{0}\right)=$ $\widehat{\mathcal{U}}_{P, m}^{0}\left(t_{1}, t_{0}\right) * \widehat{\mathcal{U}}_{P, m}\left(t_{1}, t_{0}\right)$ :

$$
\begin{aligned}
\widehat{\Omega}_{P, m}^{0}\left(t_{1}, t_{0}\right) & =1+(-i) \int_{t_{0}}^{t_{1}} d s_{1} \widehat{V}_{P}\left(s_{1}\right)+(-i)^{2} \int_{t_{0}}^{t_{1}} d s_{1} \widehat{V}_{P}\left(s_{1}\right) \int_{t_{0}}^{s_{1}} d s_{2} \widehat{V}_{P}\left(s_{2}\right) \\
& +(-i)^{3} \int_{t_{0}}^{t_{1}} d s_{1} \widehat{V}_{P}\left(s_{1}\right) \int_{t_{0}}^{s_{1}} d s_{2} \widehat{V}_{P}\left(s_{2}\right) \int_{t_{0}}^{s_{2}} d s_{3} \widehat{V}_{P}\left(s_{3}\right) \widehat{\Omega}_{P, m}^{0}\left(s_{3}, t_{0}\right) \\
& :=1+\widehat{\Omega}_{P, m}^{0,(1)}\left(t_{1}, t_{0}\right)+\widehat{\Omega}_{P, m}^{0,(2)}\left(t_{1}, t_{0}\right)+\mathcal{R}_{3},
\end{aligned}
$$


where $\widehat{V}_{P}(t)=\widehat{\mathcal{U}}_{P, m}^{0}\left(t, t_{0}\right)^{*} \widehat{V}_{P} \widehat{\mathcal{U}}_{P, m}^{0}\left(t, t_{0}\right)$. We compute explicitely the first and second order terms while the remainder $\mathcal{R}_{3}$ is estimated using Theorem 7.3.2 from Appendix 7.3. That is, we use (7.3.19) for $p=3$ which gives, with $m$ large enough

$$
\left\|\mathcal{R}_{3}\right\| \leq C_{m_{0}}\|V\|_{r}^{3} \frac{\log ^{2}\langle m\rangle}{\langle m\rangle^{\frac{3}{2}+3 r}} .
$$

As for the first two terms in the Dyson expansion by direct computation we have:

$$
\sum_{k=0}^{2} \widehat{\Omega}_{P, m}^{0,(k)}\left(t_{1}, t_{0}\right)=\left(\begin{array}{cc}
1-\frac{|\hat{V}(2(m-1))|^{2}}{m-1} c_{2, m}\left(t_{1}, t_{0}\right) & -\frac{\hat{V}(2(m-1))}{\sqrt{m-1}} \overline{c_{1, m}\left(t_{1}, t_{0}\right)} \\
\frac{\hat{V}(2(m-1))}{\sqrt{m-1}} c_{1, m}\left(t_{1}, t_{0}\right) & 1-\frac{|\hat{V}(2(m-1))|^{2}}{m-1} c_{2, m}\left(t_{1}, t_{0}\right)
\end{array}\right),
$$

with $c_{1, m}\left(t_{1}, t_{0}\right)$ and $c_{2, m}\left(t_{1}, t_{0}\right)$ as introduced in Thm. 5.1.

The expression (5.46) together with the estimate (5.45) allow to compute approximately $\widehat{\mathcal{U}}_{P, m}$ with the help of the following formula for $B_{m}(t)$ (see (5.35) and (5.14) $-($ (5.16) $)$ :

$$
B_{m}(t)=\left(\begin{array}{cc}
\gamma_{1, m}(t) & \Delta_{m}(t) \\
\bar{\Delta}_{m}(t) & \gamma_{2, m}(t)
\end{array}\right)
$$

Later on we shall need the the following expansion of $B_{m}^{\text {int }}$ :

$$
\begin{aligned}
B_{m}^{\mathrm{int}}(t) & =\widehat{\mathcal{U}}_{P, m}\left(t, t_{0}\right)^{*} B_{m}(t) \widehat{\mathcal{U}}_{P, m}\left(t, t_{0}\right) \\
& =\left(\widehat{\mathcal{U}}_{P, m}^{0}\left(t, t_{0}\right)\left(1+(-i) \int_{t_{0}}^{t} d s \hat{V}_{P}(s) \widehat{\Omega}_{P, m}^{0}\left(s, t_{0}\right)\right)\right)^{*} B_{m}(s) \widehat{\mathcal{U}}_{P, m}^{0}\left(t, t_{0}\right) \\
& \times\left(1+(-i) \int_{t_{0}}^{t} d s \hat{V}_{P}(s) \widehat{\Omega}_{P, m}^{0}\left(s, t_{0}\right)\right) .
\end{aligned}
$$

The term $(-i) \int_{t_{0}}^{t} d s \hat{V}_{P}(s) \widehat{\Omega}_{P, m}^{0}\left(s, t_{0}\right)$ is actually the remainder of order 1 from the Dyson expansion of $\widehat{\Omega}_{P, m}^{0}\left(s, t_{0}\right)$. Consequently we denote this quantity $\mathcal{R}_{1}$ and estimate it by (7.3.19) (for $m$ large enough) as

$$
\left\|\mathcal{R}_{1}\right\| \leq C_{m_{0}}\|V\|_{r} \frac{\log \langle m\rangle}{\langle m\rangle^{\frac{1}{2}+r}} .
$$

Using that $B_{m}(t)=\mathcal{O}_{r}\left((b(m))^{2}\langle m\rangle\right)$ and (5.48) we have

$$
B_{m}^{\text {int }}(t)=\widehat{\mathcal{U}}_{P, m}^{0}\left(t, t_{0}\right)^{*} B_{m}(t) \widehat{\mathcal{U}}_{P, m}^{0}\left(t, t_{0}\right)+\mathcal{O}_{r}\left(\frac{\log ^{5}\langle m\rangle}{\langle m\rangle^{\min \left\{\frac{3}{2}+r, \frac{3}{2}+5 r\right\}}}\right) \text {, as } m \rightarrow \infty \text {. }
$$

To write down $\mathcal{U}_{\mathrm{eff}, m}\left(t_{1}, t_{0}\right)$ we use (5.33), (5.36), (5.39), (5.41), (5.43), (5.44), (5.46) and (5.49):

$$
\begin{aligned}
\mathcal{U}_{\mathrm{eff}, m}\left(t_{1}, t_{0}\right) & =e^{-i \theta_{m}\left(t_{1}, t_{0}\right)}\left(\begin{array}{cc}
1-\frac{|\hat{V}(2(m-1))|^{2}}{m-1} c_{2, m}\left(t_{1}, t_{0}\right) & -\frac{\hat{V}(2(m-1))}{\sqrt{m-1}} \overline{c_{1, m}\left(t_{1}, t_{0}\right)} \\
\frac{\hat{V}(2(m-1))}{\sqrt{m-1}} \bar{c}_{1, m}\left(t_{1}, t_{0}\right) & 1-\frac{|\hat{V}(2(m-1))|^{2}}{m-1} c_{2, m}\left(t_{1}, t_{0}\right)
\end{array}\right) \\
& -i\left(\begin{array}{cc}
\omega_{1, m} & \overline{\lambda_{m}} \\
\lambda_{m} & \omega_{2, m}
\end{array}\right)+\mathcal{R}_{\mathrm{eff}, m} .
\end{aligned}
$$


The remainder $\mathcal{R}_{\text {eff }, m}$ takes into account all the errors involved during the computation of the effective evolution in the subspace $Q_{m} \mathcal{H}$ :

$$
\begin{aligned}
\left\|\mathcal{R}_{\text {eff }, m}\right\| & \left.\leq 2\left\|\mathcal{R}_{1}\right\| \cdot\left\|B_{m}\right\|+\left\|\mathcal{R}_{3}\right\|+\| B_{m}\right)\left\|^{2}+\right\| \Delta H_{\text {eff }, m} \| \\
& =\mathcal{O}_{r}\left(\frac{\log ^{8}\langle m\rangle}{\langle m\rangle^{\min \left\{2+8 r, \frac{3}{2}+5 r, \frac{3}{2}+r, 2\right\}}}\right) .
\end{aligned}
$$

Making the necessary identifications in (5.50) one finds that this expression coincides with the one given in Lemma 5.3 which is now proven.

Remark 5.4: Let us justify why we kept explicitely the matrix elements of $B_{m}(t)$ in the effective evolution. First we point out, without givind details, that one can obtain sharp estimates on $\Delta_{m}$ and $\gamma$ 's as in Eqs.(10) and (11) from [3], namely $(j=1,2)$

$$
\sup _{t \in I_{0}}\left|\Delta_{m}(t)\right| \leq \frac{C\|V\|_{r}^{2}}{\langle m\rangle^{1+r}}, \quad \sup _{t \in I_{0}}\left|\gamma_{j, m}(t)\right| \leq \frac{C\|V\|_{r}^{2}}{\langle m\rangle^{\min \{2,1+2 r\}}} .
$$

Secondly, observe that from these estimates one cannot conclude that the diagonal contribution of $B_{m}(t)$ is smaller that the second terms from the Landau-Zener coefficients $\alpha_{2 m-2}, \alpha_{2 m-2}^{\prime}$.

To obtain $\tilde{\mathcal{U}}_{\text {eff }, m}\left(t_{2}, t_{1}\right)$ we have to follow the same steps as above, using the effective Hamiltonian $\tilde{H}_{\text {eff }, m}$. Without giving explicit calculations we summarise the results and the notations. Up to errors of order $\mathcal{O}_{r}\left(\langle m\rangle^{\min \{2,2+8 r\}}\right)$ as $m \rightarrow \infty$ :

$$
\begin{aligned}
\tilde{\mathcal{U}}_{\mathrm{eff}, m}\left(t_{2}, t_{1}\right) & =e^{-i \tilde{\theta}_{m}\left(t_{2}, t_{1}\right)}\left(\begin{array}{cc}
1-\frac{|\hat{V}(2 m-1)|^{2}}{m-\frac{1}{2}} \tilde{c}_{2, m}\left(t_{2}, t_{1}\right) & \left.-\frac{\hat{V}(2 m-1)}{\sqrt{m-\frac{1}{2}}} \bar{c}_{1, m}\left(t_{2}, t_{1}\right)\right) \\
\frac{\hat{V}(2 m-1)}{\sqrt{m-\frac{1}{2}}} \tilde{c}_{1, m}\left(t_{2}, t_{1}\right) & 1-\frac{|\hat{V}(2 m-1)|^{2}}{m+\frac{1}{2}} \tilde{c}_{2, m}\left(t_{2}, t_{1}\right)
\end{array}\right) \\
& -i e^{-i \tilde{\theta}_{m}\left(t_{2}, t_{1}\right)}\left(\begin{array}{cc}
\tilde{\omega}_{1, m}\left(t_{2}, t_{1}\right) & \overline{\tilde{\lambda}_{m}\left(t_{2}, t_{1}\right)} \\
\tilde{\lambda}_{m}\left(t_{2}, t_{1}\right) & \tilde{\omega}_{2, m}\left(t_{2}, t_{1}\right)
\end{array}\right)
\end{aligned}
$$

the phase $\tilde{\theta}_{m}$ coeficients $\tilde{c}_{i}(m)$ being related to the ones of $\mathcal{U}_{\text {eff }, m}\left(t_{1}, t_{0}\right)$ by the relations

$$
\begin{aligned}
\tilde{\theta}_{m}\left(t_{2}, t_{1}\right) & =\theta_{m+\frac{1}{2}}\left(t_{1}, t_{0}\right) \\
\tilde{c}_{i, m}\left(t_{2}, t_{1}\right) & =c_{i, m+\frac{1}{2}}\left(t_{1}, t_{0}\right), \quad i=1,2 .
\end{aligned}
$$

$\tilde{\lambda}_{m}\left(t_{2}, t_{1}\right)$ and $\tilde{\gamma}_{1, m}(t)$ are to be computed in the same way as $\lambda_{m}\left(t_{1}, t_{0}\right)$ and $\gamma_{m}\left(t_{1}, t_{0}\right)$ (replace $\varphi_{-(m-1), 0}$ with $\varphi_{-m, 0}$ and $\hat{E}_{m, 1}(t)$ with $\tilde{\hat{E}}_{m, 1}(t)$ associated to $\tilde{Q}_{m}(t)$ ). Finally:

$$
\begin{aligned}
\tilde{\alpha}_{2 m-1}\left(t_{2}, t_{1}\right) & :=1-\frac{|\hat{V}(2 m-1)|^{2}}{m-\frac{1}{2}} \tilde{c}_{2, m}\left(t_{2}, t_{1}\right)-i \tilde{\omega}_{1, m}\left(t_{2}, t_{1}\right) \\
\tilde{\beta}_{2 m-1} & :=\frac{\hat{V}(2 m-1)}{\sqrt{m-\frac{1}{2}}} \tilde{c}_{1, m}\left(t_{2}, t_{1}\right)-i \tilde{\lambda}_{m}\left(t_{2}, t_{1}\right) .
\end{aligned}
$$


The proof of Theorem 5.1 is finished.

\section{The long time behaviour. Spectral properties}

The last part of this work is concerned with the long time behaviour of the Bloch electron. Suppose that at the time $t_{0}=-\frac{1}{4}$ the electron is in a given band (say $E_{2 n_{0}-2}\left(t_{0}\right)$ for $n_{0}$ fixed) and its wavefunction $\psi\left(t_{0}\right)=\varphi_{2 n_{0}-2}\left(t_{0}\right)$ (let us recall that $\varphi_{i}(t)$ is the eigenfunction of $H(t)$ which corresponds to the eigenvalue $\left.E_{i}(t)\right)$. We are interested in obtaining some quantitative information about $\psi\left(t_{N}\right)=U\left(t_{N}, t_{0}\right) \psi\left(t_{0}\right)$ where $t_{N}=t_{0}+\frac{N}{2}$, particular attention being payed to the case when $N$ goes to infinity.

To answer these questions we proceed as follows: the complete evolution $U\left(t_{N}, t_{0}\right)$ is written as a product of one-period evolutions $U\left(t_{l}+1, t_{l}\right)$ each of them being then reduced to the first period $I_{0} \cup I_{1}$ by using (2.27). Then we use Theorem 3.1 to approximate the one-period evolution by the adiabatic evolutions which were written explicitely (in suitable subspaces) in Theorem 5.1. As pointed out by Ao it is of particular interest to establish how far the electron goes in the energy space, otherwise stated, to say up to what band it is accelerated by the electric field. Because in the neighbour band approximation the electron can jump during one half period only up to the next band it is clear that after $N$ half periods the uppermost reachable band is $E_{2 n_{0}-2+N}\left(t_{N}\right)$. Its eigenfunction $\varphi_{2 n_{0}-2+N}\left(t_{N}\right)$ is related to $\varphi_{2 n_{0}-2+N}\left(t_{0}\right)$ by the shift operator $T$ (see (2.51) ). The long time behaviour of the Bloch electron is described in the following theorem:

Theorem 6.1: Let $r>0$ and $n_{0}$ sufficiently large. Define the so-called propagating front [2]:

$$
\mathcal{P}(N):=\left|\left\langle\varphi_{2 n_{0}-2+N}\left(t_{N}\right), U\left(t_{N}, t_{0}\right) \varphi_{2 n_{0}-2}\left(t_{0}\right)\right\rangle\right|^{2} .
$$

Then

$$
\liminf _{N \rightarrow \infty} \mathcal{P}(N)=\exp \left\{-2 \sum_{l=0}^{\infty} \frac{\left|\hat{V}\left(2 n_{0}+l-2\right)\right|^{2}}{n_{0}+\frac{l}{2}-1} c_{2, n_{0}+\frac{l}{2}}\left(t_{1}, t_{0}\right)\right\} \times(1+\mathcal{R}),
$$

with

$$
|\mathcal{R}| \leq \sum_{l=0}^{\infty} \frac{C_{V}}{\left\langle n_{0}+l-\frac{1}{2}\right\rangle^{1+2 r}}+\frac{C_{V} \log ^{8}\left\langle n_{0}\right\rangle}{\left\langle n_{0}\right\rangle^{\min \left\{1+8 r, \frac{1}{2}+5 r, \frac{1}{2}+r, 1\right\}}} \exp \left(\sum_{l=0}^{\infty} \frac{C_{V}}{\left\langle n_{0}+\frac{l}{2}-1\right\rangle^{1+2 r}}\right) .
$$

As one may guess we need first a more explicit form for the scalar product in Eq. (6.1). Consequently a preliminar result is 
Lemma 6.2: For $r>-\frac{1}{10}, n_{0}$ sufficiently large and $N$ even one gets:

$$
\begin{aligned}
& \left\langle\varphi_{2 n_{0}+N-2}\left(t_{N}\right), U\left(t_{N}, t_{0}\right) \varphi_{2 n_{0}-2}\left(t_{0}\right)\right\rangle \\
= & \prod_{l=0}^{\frac{N}{2}-1} e^{-i\left(\tilde{\theta}_{n_{0}+l}\left(t_{2}, t_{1}\right)+\theta_{n_{0}+l}\left(t_{1}, t_{0}\right)\right)} \tilde{\alpha}_{2\left(n_{0}+l\right)-1}\left(t_{2}, t_{1}\right) \alpha_{2\left(n_{0}+l\right)-2}\left(t_{1}, t_{0}\right)+\mathcal{R}(N),(6 .
\end{aligned}
$$

where the remainder $\mathcal{R}(N)$ satisfy the estimate

$$
|\mathcal{R}(N)| \leq \frac{C_{V} \log ^{8}\left\langle n_{0}\right\rangle}{\left\langle n_{0}\right\rangle^{\min \left\{1+8 r, \frac{1}{2}+5 r, \frac{1}{2}+r, 1\right\}}}
$$

A similar expression holds for $N$ odd.

Proof: The idea is to factorise $U\left(t_{N}, t_{0}\right)$ into one-period evolutions and to use the adiabatic theorem for a suitable index $m$ of the two-dimensional projector $Q_{m}(t)$ or $\tilde{Q}_{m}(t)$. By the telescoping sum rule:

$$
\prod_{l=0}^{n} a_{l}-\prod_{l=0}^{n} b_{l}=\sum_{l^{\prime}=0}^{n} \prod_{l=l^{\prime}+1}^{n} a_{l}\left(a_{l^{\prime}}-b_{l^{\prime}}\right) \prod_{l=0}^{l^{\prime}-1} b_{l}
$$

one has

$$
\begin{aligned}
& U\left(t_{N}, t_{0}\right)=\prod_{l=0}^{\frac{N}{2}-1} U\left(t_{2 l+2}, t_{2 l+1}\right) U\left(t_{2 l+1}, t_{2 l}\right) \\
= & \prod_{l=0}^{\frac{N}{2}-1} \tilde{U}_{n_{0}+l}^{A}\left(t_{2 l+2}, t_{2 l+1}\right) U_{n_{0}+l}^{A}\left(t_{2 l+1}, t_{2 l}\right) \\
+ & \sum_{l=0}^{\frac{N}{2}-1} \prod_{k=l+1}^{\frac{N}{2}-1} U\left(t_{2 k+2}, t_{2 k}\right)\left(U\left(t_{2 l+2}, t_{2 l}\right)-\tilde{U}_{n_{0}+l}^{A}\left(t_{2 l+2}, t_{2 l+1}\right) U_{n_{0}+l}^{A}\left(t_{2 l+1}, t_{2 l}\right)\right) \times \\
\times & \prod_{k=0}^{l-1} \tilde{U}_{n_{0}+k}^{A}\left(t_{2 k+2}, t_{2 k+1}\right) U_{n_{0}+k}^{A}\left(t_{2 k+1}, t_{2 k}\right) \\
= & \prod_{l=0}^{\frac{N}{2}-1} \tilde{U}_{n_{0}+l}^{A}\left(t_{2 l+2}, t_{2 l+1}\right) U_{n_{0}+l}^{A}\left(t_{2 l+1}, t_{2 l}\right)+\mathcal{R}_{\mathrm{ad}}(N) .
\end{aligned}
$$

The property $T^{*} U(t, s) T=U(t+1, s+1)$ leads to

$$
\begin{aligned}
\mathcal{R}_{\mathrm{ad}}(N):= & \left.\sum_{l=0}^{\frac{N}{2}-1} U\left(t_{N}, t_{2 l+2}\right)\left(T^{*}\right)^{l}\left(U\left(t_{2}, t_{0}\right)\right)-\tilde{U}_{n_{0}+l}^{A}\left(t_{2}, t_{1}\right) U_{n_{0}+l}^{A}\left(t_{1}, t_{0}\right)\right) T^{l} \times \\
& \times \prod_{k=0}^{l-1}\left(T^{*}\right)^{k} \tilde{U}_{n_{0}+k}^{A}\left(t_{2}, t_{1}\right) U_{n_{0}+k}^{A}\left(t_{1}, t_{0}\right) T^{k} .
\end{aligned}
$$


We pass now to the estimation of $\mathcal{R}_{\text {ad }}(N)$. Clearly do the presence of many unitarities one gets at once from the adiabatic theorem:

$$
\begin{aligned}
\left\|\mathcal{R}_{\mathrm{ad}}(N)\right\| & \leq \sum_{l=0}^{\frac{N}{2}-1}\left\|U\left(t_{2}, t_{0}\right)-\tilde{U}_{n_{0}+l}^{A}\left(t_{2}, t_{1}\right) U_{n_{0}+l}^{A}\left(t_{1}, t_{0}\right)\right\| \\
& \leq \sum_{l=0}^{\frac{N}{2}-1}\left(\left\|U\left(t_{2}, t_{1}\right)-\tilde{U}_{n_{0}+l}^{A}\left(t_{2}, t_{1}\right)\right\|+\left\|U\left(t_{1}, t_{0}\right)-U_{n_{0}+l}^{A}\left(t_{1}, t_{0}\right)\right\|\right) \\
& \leq \sum_{l=0}^{\frac{N}{2}-1} 2 C_{V} b\left(n_{0}+l\right)\left\langle n_{0}+l\right\rangle^{-1} .
\end{aligned}
$$

We concentrate in the following on the term

$$
\begin{aligned}
& \prod_{l=0}^{\frac{N}{2}-1} \tilde{U}_{n_{0}+l}^{A}\left(t_{2 l+2}, t_{2 l+1}\right) U_{n_{0}+l}^{A}\left(t_{2 l+1}, t_{2 l}\right) P_{2 n_{0}-2}\left(t_{0}\right) \\
= & \sum_{j_{\frac{N}{2}}=1}^{\infty} P_{j_{\frac{N}{2}}}\left(t_{N}\right) \times \\
& \times \prod_{l=0}^{\frac{N}{2}-1}\left(\tilde{U}_{n_{0}+l}^{A}\left(t_{2 l+2}, t_{2 l+1}\right) \sum_{\tilde{j}_{l}=1}^{\infty} P_{\tilde{j}_{l}}\left(t_{2 l+1}\right) U_{n_{0}+l}^{A}\left(t_{2 l+1}, t_{2 l}\right) \sum_{j_{l}=1}^{\infty} P_{j_{l}}\left(t_{2 l}\right)\right) P_{2 n_{0}-2}\left(t_{0}\right) \\
= & \sum_{j_{\frac{N}{2}}, j_{\frac{N}{2}-1}, \tilde{j}_{\frac{N}{2}-1}, \ldots j_{0}, \tilde{j}_{0}} P_{j_{\frac{N}{2}}}\left(t_{N}\right) \times \\
& \times\left(\prod_{l=0}^{\frac{N}{2}-1} \tilde{U}_{n_{0}+l}^{A}\left(t_{2 l+2}, t_{2 l+1}\right) P_{\tilde{j}_{l}}\left(t_{2 l+1}\right) U_{n_{0}+l}^{A}\left(t_{2 l+1}, t_{2 l}\right) P_{j_{l}}\left(t_{2 l}\right)\right) P_{2 n_{0}-2}\left(t_{0}\right),
\end{aligned}
$$

where all the j's indices run for the moment from 1 to $\infty$. However since $U_{n}^{A}$ and $\tilde{U}_{n}^{A}$ leave invariant $\operatorname{Ran} Q_{n}$ and $\operatorname{Ran} \tilde{Q}_{n}$ and since for any $l \in \mathbb{Z}$

$$
\begin{aligned}
& Q_{n}\left(t_{l}\right)=P_{2 n-2}\left(t_{l}\right)+P_{2 n-1}\left(t_{l}\right) \\
& \tilde{Q}_{n}\left(t_{l}\right)=P_{2 n-1}\left(t_{l}\right)+P_{2 n}\left(t_{l}\right),
\end{aligned}
$$

we know that the only indices which contribute are the ones of the set $J_{2 n_{0}-2}$ where we define $J_{n}$ as follows:

$J_{n}:=\left\{\left(j_{\frac{N}{2}}, \tilde{j}_{\frac{N}{2}-1}, j_{\frac{N}{2}-1}, \ldots, \tilde{j}_{0}, j_{0}\right), j_{0}=2 n_{0}-2, \forall l, \tilde{j}_{l}=j_{l}\right.$ or $j_{l}+1 ; j_{l+1}=\tilde{j}_{l}$ or $\left.\tilde{j}_{l}+1\right\}$.

Then ( we drop the times variables in $U^{A}$ and $\tilde{U}^{A}$ since their values are clear from the context)

$$
\left(\prod_{l=0}^{\frac{N}{2}-1} \tilde{U}_{n_{0}+l}^{A} U_{n_{0}+l}^{A}\right) P_{2 n_{0}-2}=\sum_{J_{2 n_{0}-2}} P_{j_{\frac{N}{2}}} \prod_{l=0}^{\frac{N}{2}-1} \tilde{U}_{n_{0}+l}^{A} P_{\tilde{j}_{l}} U_{n_{0}+l}^{A} P_{j_{l}} .
$$


Clearly if we force $j_{\frac{N}{2}}$ to be equal to $2 n_{0}+N-2$ by multiplying on the left by $P_{2 n_{0}+N-2}$ it remains only one "path" in this sum. Using (2.27) (2.51), (5.3) and (5.4) one may write

$$
\begin{aligned}
&\left\langle\varphi_{2 n_{0}+N-2}\left(t_{N}\right), \prod_{l=0}^{\frac{N}{2}-1} \tilde{U}_{n_{0}+l}^{A}\left(t_{2 l+2}, t_{2 l+1}\right) U_{n_{0}+l}^{A}\left(t_{2 l+1}, t_{2 l}\right) \varphi_{2 n_{0}-2}\left(t_{0}\right)\right\rangle \\
&=\left\langle\varphi_{2 n_{0}+N-2}\left(t_{N}\right), P_{2 n_{0}+N-2}\left(t_{N}\right) \times\right. \\
&\left.\times \prod_{l=0}^{\frac{N}{2}-1} \tilde{U}_{n_{0}+l}^{A}\left(t_{2 l+2}, t_{2 l+1}\right) P_{2 n_{0}+2 l-1}\left(t_{2 l+1}\right) U_{n_{0}+l}^{A}\left(t_{2 l+1}, t_{2 l}\right) P_{2 n_{0}+2 l-2}\left(t_{2 l}\right) \varphi_{2 n_{0}-2}\left(t_{0}\right)\right\rangle \\
&=\left\langle\varphi_{2 n_{0}+N-2}\left(t_{2}\right), T^{\frac{N}{2}-1} P_{2 n_{0}+N-2}\left(t_{N}\right) \times\right. \\
& \times\left.\prod_{l=0}^{\frac{N}{2}-1}\left(T^{*}\right)^{l} \tilde{U}_{n_{0}+l}^{A}\left(t_{2}, t_{1}\right) P_{2 n_{0}+2 l-1}\left(t_{1}\right) U_{n_{0}+l}^{A}\left(t_{1}, t_{0}\right) P_{2 n_{0}+2 l-2}\left(t_{0}\right) T^{l} \varphi_{2 n_{0}-2}\left(t_{0}\right)\right\rangle \\
&= \prod_{l=0}^{\frac{N}{2}-1} \tilde{S}_{2 n_{0}+2 l, 2 n_{0}+2 l-1}^{n_{0}+l}\left(t_{2}, t_{1}\right) S_{2 n_{0}+2 l-1,2 n_{0}+2 l-2}^{n_{0}+l}\left(t_{1}, t_{0}\right) \\
& \quad: \prod_{l=0}^{\frac{N}{2}-1} e^{-i \tilde{\theta}_{n_{0}+l}\left(t_{2}, t_{1}\right)} \tilde{\alpha}_{2 n_{0}+2 l-1}\left(t_{2}, t_{1}\right) e^{-i \theta_{n_{0}+l}\left(t_{1}, t_{0}\right)} \alpha_{2 n_{0}+2 l-2}\left(t_{1}, t_{0}\right)+\mathcal{R}_{\mathrm{eff}}(N), \\
&
\end{aligned}
$$

where

$$
\begin{aligned}
\left|\mathcal{R}_{\mathrm{eff}}(N)\right| & \leq \sum_{l=0}^{\frac{N}{2}-1}\left(2\left|\mathcal{R}_{\mathrm{eff}, n_{0}+l}\right|+\left|\mathcal{R}_{\mathrm{eff}, n_{0}+l}\right|^{2}\right) \\
& \leq \sum_{l=0}^{\infty}\left(2\left|\mathcal{R}_{\mathrm{eff}, n_{0}+l}\right|+\left|\mathcal{R}_{\mathrm{eff}, n_{0}+l}\right|^{2}\right) \\
& =\frac{C_{V} \log ^{8}\left\langle n_{0}\right\rangle}{\left\langle n_{0}\right\rangle^{\min \left\{1+8 r, \frac{1}{2}+5 r, \frac{1}{2}+r, 1\right\}}} .
\end{aligned}
$$

Defining

$$
\mathcal{R}(N):=\mathcal{R}_{\text {eff }}(N)+\left\langle\varphi_{2 n_{0}+N-2}, \mathcal{R}_{\text {ad }}(N) \varphi_{2 n_{0}-2}\right\rangle
$$

the lemma follows immediately from the estimates of $\mathcal{R}_{\text {ad }}(N)$ and $\mathcal{R}_{\text {eff }}(N)$.

Proof of Theorem 6.1: Let

$$
\mathcal{A}(N):=\prod_{l=0}^{\frac{N}{2}-1} e^{-i\left(\tilde{\theta}_{n_{0}+l}\left(t_{2}, t_{1}\right)+\theta_{n_{0}+l}\left(t_{1}, t_{0}\right)\right)} \tilde{\alpha}_{2 n_{0}+2 l-1}\left(t_{2}, t_{1}\right) \alpha_{2 n_{0}+2 l-2}\left(t_{1}, t_{0}\right) .
$$

Then by Lemma 6.2 the propagating front reads as:

$$
\mathcal{P}(N)=|\mathcal{A}(N)|^{2} \cdot\left|1+\frac{\mathcal{R}(N)}{\mathcal{A}(N)}\right|^{2}
$$


Replacing $\alpha_{2 m-2}\left(t_{1}, t_{0}\right)$ and $\tilde{\alpha}_{2 m-1}\left(t_{2}, t_{1}\right)$ for $m=n_{0}+k$ it follows that (we omit the time arguments for the simplicity of writing)

$$
\begin{aligned}
& |\mathcal{A}(N)|=\prod_{l=0}^{\frac{N}{2}-1}\left|\tilde{\alpha}_{2 n_{0}+2 l-1} \alpha_{2 n_{0}+2 l-2}\right| \\
& =\prod_{l=0}^{\frac{N}{2}-1}\left(\left(1-\frac{\left|\hat{V}\left(2 n_{0}+2 l-1\right)\right|^{2}}{n_{0}+l-\frac{1}{2}} \tilde{c}_{2, n_{0}+l}\right)^{2}+\tilde{\omega}_{1, n_{0}+l}^{2}\right)^{\frac{1}{2}} \times \\
& \times\left(\left(1-\frac{\left|\hat{V}\left(2\left(n_{0}+l-1\right)\right)\right|^{2}}{n_{0}+l-1} c_{2, n_{0}+l}\right)^{2}+\omega_{1, n_{0}+l}^{2}\right)^{\frac{1}{2}} \\
& =\exp \left\{\frac{1}{2} \sum_{l=0}^{\frac{N}{2}-1} \log \left(\left(1-\frac{\left|\hat{V}\left(2 n_{0}+2 l-1\right)\right|^{2}}{n_{0}+l-\frac{1}{2}} \tilde{c}_{2, n_{0}+l}\right)^{2}+\tilde{\omega}_{1, n_{0}+l}^{2}\right)\right\} \times \\
& \times \exp \left\{\frac{1}{2} \sum_{l=0}^{\frac{N}{2}-1} \log \left(\left(1-\frac{\left|\hat{V}\left(2\left(n_{0}+l-1\right)\right)\right|^{2}}{n_{0}+l-1} c_{2, n_{0}+l}\right)^{2}+\omega_{1, n_{0}+l}^{2}\right)\right\} \\
& =\exp \left\{-\sum_{l=0}^{N-1} \frac{\left|\hat{V}\left(2 n_{0}+l-2\right)\right|^{2}}{n_{0}+\frac{l}{2}-1} c_{2, n_{0}+\frac{l}{2}}\right\} \times\left(1+\mathcal{O}\left(\sum_{l=0}^{\infty} \frac{1}{\left\langle n_{0}+l-\frac{1}{2}\right\rangle^{1+2 r}}\right)\right)
\end{aligned}
$$

where we used estimates of the following type (similar bounds are found for the sums containing $\omega_{1, n_{0}+l}^{2}$ and $\left.\tilde{\omega}_{1, n_{0}+l}^{2}\right)$ and the relation $\tilde{c}_{i, m}\left(t_{2}, t_{1}\right)=c_{i, m+\frac{1}{2}}\left(t_{1}, t_{0}\right)$ :

$$
\sum_{l=0}^{\frac{N}{2}-1}\left|\frac{\left|\hat{V}\left(2 n_{0}+2 l-1\right)\right|^{2}}{n_{0}+l-\frac{1}{2}} \tilde{c}_{2, n_{0}+l}\right| \leq \sum_{l=0}^{\infty}\left|\frac{C_{V}}{\left\langle n_{0}+l-\frac{1}{2}\right\rangle^{1+2 r}}\right|
$$

Thus

$$
\lim _{N \rightarrow \infty}\left|\frac{\mathcal{R}(N)}{\mathcal{A}(N)}\right|=:|\mathcal{R}| \leq \frac{C_{V} \log ^{8}\left\langle n_{0}\right\rangle}{\left\langle n_{0}\right\rangle^{\min \left\{1+8 r, \frac{1}{2}+5 r, \frac{1}{2}+r, 1\right\}}} \exp \left(\sum_{l=0}^{\infty} \frac{C_{V}}{\left\langle n_{0}+\frac{l}{2}-1\right\rangle^{1+2 r}}\right) .
$$

The proof of theorem is done.

Remark 6.3: Since as long as $r>0$ the series that appears in Eq. (6.2) both in the exponential and in the error term converges the propagating front does not vanish. This means that the electron is always accelerated and it escapes at infinity in the energy space, provided that it is initially prepared in a band with sufficiently energy. Thus we recovered rigorously the Ao's result. We end the paper with a result on the spectral properties of the Stark-Wannier operator:

Corollary 6.4 For $r>0$ one has $\sigma_{\text {cont }}\left(H^{S W}\right) \neq \emptyset$.

Proof of Corollary 6.4: Let $\phi \in \mathcal{H}_{\mathrm{pp}}\left(H^{S W}\right)$ and $\Pi_{N}$ a family of bounded operators that goes strongly to zero when $N \rightarrow \infty$. Then it is known (see [14]) that

$$
\lim _{N \rightarrow \infty} \sup _{ \pm t>0}\left\|\Pi_{N} e^{-i t H^{S W}} \phi\right\|=0 .
$$


In particular, for $t_{N}:=t_{0}+\frac{N}{2}$

$$
\lim _{N \rightarrow \infty}\left\|\Pi_{N} e^{-i\left(t_{N}-t_{0}\right) H^{S W}} \phi\right\|=0
$$

The strategy of the proof consists in writing $\Pi_{N} e^{-i\left(t_{N}-t_{0}\right) H^{S W}} \phi$ in the Fourier-Bloch representation and then to express the result in terms of the propagating front $\mathcal{P}(N)$ which was computed in Thm. 6.1. Using (2.23), writing (see (2.24)

$$
\begin{aligned}
U\left(k, t_{N}, t_{0}\right) & =U\left(0, t_{N}+k, t_{0}+k\right)=: U\left(t_{N}+k, t_{0}+k\right) \\
& =U\left(t_{N}+k, t_{N}\right) U\left(t_{N}, t_{0}\right) U\left(t_{0}, t_{0}+k\right)
\end{aligned}
$$

and chosing

$$
\phi:=e^{i t_{0} X} S^{*}\left(\int_{[0,1)}^{\oplus} d k U\left(t_{0}, t_{0}+k\right) \varphi_{2 n_{0}-2}\left(t_{0}\right)\right)
$$

one has

$$
\begin{aligned}
& \left\|\Pi_{N} e^{-i\left(t_{N}-t_{0}\right) H^{S W}} \phi\right\| \\
= & \left\|S e^{-i t_{N} X} \Pi_{N} e^{i t_{N} X} S^{*}\left(\int_{[0,1)}^{\oplus} d k U\left(t_{N}+k, t_{N}\right) U\left(t_{N}, t_{0}\right) \varphi_{2 n_{0}-2}\left(t_{0}\right)\right)\right\| .
\end{aligned}
$$

Now we chose $\Pi_{N}$ such that:

$$
S e^{-i t_{N} X} \Pi_{N} e^{i t_{N} X} S^{*}\left(\int_{[0,1)}^{\oplus} d k U\left(t_{N}+k, t_{N}\right)\right)=\left(\int_{[0,1)}^{\oplus} d k P_{2 n_{0}-2+N}\left(t_{N}\right)\right) .
$$

Let us show that $\Pi_{N}$ goes strongly to zero as $N$ goes to infinity. Using the identity

$$
S e^{-i n X} S^{*}=\mathrm{id} \otimes T^{-n}, \quad \forall n \in \mathbb{Z}
$$

it turns out that $\Pi_{N}$ is given by

$$
\begin{aligned}
\Pi_{N} & :=e^{i t_{N} X} S^{*}\left(\int_{[0,1)}^{\oplus} d k P_{2 n_{0}-2+N}\left(t_{N}\right) U\left(t_{N}, t_{N}+k\right)\right) S e^{-i t_{N} X} \\
& =e^{i t_{0} X} S^{*}\left(\int_{[0,1)}^{\oplus} d k T^{\frac{N}{2}} P_{2 n_{0}-2+N}\left(t_{N}\right) U\left(t_{N}, t_{N}+k\right) T^{-\frac{N}{2}}\right) S e^{-i t_{0} X} \\
& =e^{i t_{0} X} S^{*}\left(\int_{[0,1)}^{\oplus} d k P_{2 n_{0}-2+N}\left(t_{0}\right) U\left(t_{0}, t_{0}+k\right)\right) S e^{-i t_{0} X} .
\end{aligned}
$$

Then clearly

$$
s-\lim _{N \rightarrow \infty} \Pi_{N}=0 .
$$

Finally observe that from (6.11) and (6.12)

$$
\left\|\Pi_{N} e^{-i\left(t_{N}-t_{0}\right) H^{S W}} \phi\right\|^{2}=\left\|P_{2 n_{0}-2+N}\left(t_{N}\right) U\left(t_{N}, t_{0}\right) \varphi_{2 n_{0}-2}\left(t_{0}\right)\right\|^{2}=\mathcal{P}(N) .
$$


However, from Thm.6.1 we know that for $n_{0}$ sufficiently large and $r>0$ the propagating front $\mathcal{P}(N)$ does not vanish when $N \rightarrow \infty$ so $\phi$ must have a part in $\mathcal{H}_{\text {cont }}\left(H^{S W}\right)$.

When applied to the driven quantum ring problem the existence of a propagating front gives at once:

Corollary 6.5 Let $r>0$ and $\varphi\left(t_{0}=-\frac{1}{4}\right)=\varphi_{2 n_{0}-2}\left(t_{0}\right), \varphi\left(t_{N}=t_{0}+\frac{N}{2}\right)=$ $U\left(t_{N}, t_{0}\right) \varphi\left(t_{0}\right)$. Then

$$
\left\langle\varphi\left(t_{N}\right), H(t) \varphi\left(t_{N}\right)\right\rangle \geq\left(1+\mathcal{O}_{r}\left(\frac{1}{\left\langle n_{0}\right\rangle^{r}}\right)\right) \cdot t^{2} .
$$

We end up this section of applications by showing that there is no localization in momentum space for appropriate initial conditions.

Corollary 6.6 Let $F_{D}(I)$ be the spectral projection of $D:=-i \partial_{x}$ on the intervalI $:=$ $\left[n_{0}, n_{0}+1\right)$. Then for all $V$ such that $r>0$ one has

$$
\lim _{n_{0} \rightarrow \infty} \lim _{N \rightarrow \infty}\left\|F_{D}\left(I+t_{N}\right) e^{-i\left(t_{n}-t_{0}\right) H^{S W}} F_{D}\left(I+t_{0}\right)\right\|=1 .
$$

Proof: One can check that $S D S^{\star}$ is simply the multiplication by $k+n$; thus $S F_{D}(I) S^{\star}=1 \otimes P_{n_{0}, 0}$. One has successively:

$$
\begin{aligned}
& \left\|F_{D}\left(I+t_{N}\right) e^{-i\left(t_{N}-t_{0}\right) H^{S W}} F_{D}\left(I+t_{0}\right)\right\| \\
= & \left.\left.\left\|F_{D}\left(I+t_{N}\right) e^{i t_{N} X} S^{\star} \int_{[0,1)}^{\oplus} d k U\left(t_{N}+k, t_{0}+k\right) S e^{-i t_{0} X} F_{D}\left(I+t_{0}\right)\right\| \quad \text { (see (2.23) }\right)\right) \\
= & \left\|S e^{-i t_{N} X} F_{D}\left(I+t_{N}\right) e^{i t_{N} X} S^{\star} \int_{[0,1)}^{\oplus} d k U\left(t_{N}+k, t_{0}+k\right) S e^{-i t_{0} X} F_{D}\left(I+t_{0}\right) e^{i t_{0} X} S^{\star}\right\| \\
= & \left\|S F_{D}(I) S^{\star} \int_{[0,1)}^{\oplus} d k U\left(t_{N}+k, t_{0}+k\right) S F_{D}(I) S^{\star}\right\| \\
= & \left\|1 \otimes P_{n_{0}, 0} \int_{[0,1)}^{\oplus} d k U\left(t_{N}+k, t_{0}+k\right) 1 \otimes P_{n_{0}, 0}\right\|=\sup _{k \in[0,1)}\left\|P_{n_{0}, 0} U\left(t_{N}+k, t_{0}+k\right) P_{n_{0}, 0}\right\| \\
\geq & \left.\left\|P_{n_{0}, 0} U\left(t_{N}, t_{0}\right) P_{n_{0}, 0}\right\|=\left\|P_{2 n_{0}+N}^{0}\left(t_{N}\right) U\left(t_{N}, t_{0}\right) P_{2 n_{0}+N}^{0}\left(t_{0}\right)\right\| \quad \text { (see (2.32) }\right) \\
= & \left\|P_{2 n_{0}+N}\left(t_{N}\right) U\left(t_{N}, t_{0}\right) P_{2 n_{0}+N}\left(t_{0}\right)\right\|+\mathcal{O}_{r}\left(b\left(n_{0}+1\right)\right) \quad(\text { see Corollary 2.6) } \\
= & \mathcal{P}(N+1)+\mathcal{O}_{r}\left(b\left(n_{0}+1\right)\right) .
\end{aligned}
$$

Using theorem 6.1, the rest is now clear.

\section{Appendices}

\subsection{The key estimate}

Proof of Lemma 2.3: 
In the following we shall use the notation $\alpha:=\max \{0,-r\}$. We start by estimating $\sup _{t \in I_{0}} \sup _{z \in \Gamma_{m}}\left\|K\left(\left(d_{m}+i y\right), t\right)\right\|$ :

$$
\begin{aligned}
& \sup _{t \in I_{0}} \sup _{z \in \Gamma_{m}}\left\|K\left(\left(d_{m}+i y\right), t\right)\right\|_{H S} \leq\|V\|_{r}\left(\frac{\sqrt{5}}{2}\right)^{\alpha} \sup _{t \in I_{0}} \sum_{n \in \mathbb{Z}} \frac{\langle n\rangle^{2 \alpha}}{\left|(n+t)^{2}-d_{m}-i y\right|} \\
\leq & \|V\|_{r}\left(\frac{\sqrt{5}}{2}\right)^{\alpha} \sup _{t \in I_{0}} \sum_{n \in \mathbb{Z}} \frac{\langle n\rangle^{2 \alpha}}{\left|(n+t)^{2}-d_{m}\right|} \leq 4\|V\|_{r}\left(\frac{\sqrt{5}}{2}\right)^{\alpha} \sum_{n \in \mathbb{Z}} \frac{\langle n\rangle^{2 \alpha}}{\left|n^{2}-d_{m}\right|} \\
= & \|V\|_{r}\left(\frac{\sqrt{5}}{2}\right)^{\alpha}\left\{\frac{1}{d_{m}}+2 \sum_{n \geq 1} \frac{\langle n\rangle^{2 \alpha}}{\left|n^{2}-d_{m}\right|}\right\}
\end{aligned}
$$

where the time dependence of $K\left(\left(d_{m}+i y\right), t\right)$ was eliminated by using the inequality (proved bellow )

$$
\sup _{t \in I_{0}}\left|(n+t)^{2}-d_{m}\right| \geq \frac{1}{4}\left|n^{2}-d_{m}\right| .
$$

The next step is to estimate the sum from the RHS of (7.1.1). Let us assume that $m>1$ (the particular case $m=1$ will be discussed separately). Then approximating the sum with integrals one has

$$
\begin{aligned}
\sum_{n \geq 1} \frac{\langle m\rangle^{2 \alpha}}{\left|n^{2}-d_{m}\right|} & \leq \int_{1}^{m-1} \frac{\langle x\rangle^{2 \alpha}}{d_{m}-x^{2}} d x+\frac{\langle m-1\rangle^{2 \alpha}}{\frac{1}{2}\left(2 m-\frac{3}{2}\right)}+\frac{\langle m\rangle^{2 \alpha}}{m-\frac{1}{4}}+\int_{m}^{\infty} \frac{\langle x\rangle^{2 \alpha}}{x^{2}-d_{m}} \\
& =: S_{1}+S_{2}+S_{3}+S_{4} .
\end{aligned}
$$

Since $\langle x\rangle$ is an increasing function we can estimate the term $S_{1}$ as follows

$$
\begin{aligned}
S_{1} & \leq\langle m-1\rangle^{2 \alpha} \int_{1}^{m-1} \frac{d x}{d_{m}-x^{2}} \\
& =\frac{\langle m-1\rangle^{2 \alpha}}{2 \sqrt{d_{m}}} \log \left(\frac{\sqrt{d_{m}}+m-1}{\sqrt{d_{m}}-(m-1)} \cdot \frac{\sqrt{d_{m}}-1}{\sqrt{d_{m}}+1}\right) \\
& \leq \frac{\langle m\rangle^{2 \alpha}}{2 \sqrt{d_{m}}} \log \left(\frac{m+\sqrt{d_{m}}}{m-\sqrt{d_{m}}}\right) .
\end{aligned}
$$

To obtain the last line we used the following estimate (valid for $m \geq 2$ ):

$$
\begin{aligned}
& \frac{\sqrt{d_{m}}+m-1}{\sqrt{d_{m}}-(m-1)} \cdot \frac{\sqrt{d_{m}}-1}{\sqrt{d_{m}}+1}=\frac{2 m-\frac{3}{2}}{\frac{1}{2}} \cdot \frac{m-\frac{3}{2}}{m+\frac{1}{2}} \\
& \leq \frac{2 m-\frac{1}{2}}{\frac{1}{2}}=\frac{m+\sqrt{d_{m}}}{m-\sqrt{d_{m}}} .
\end{aligned}
$$

The next two terms are easier:

$$
\begin{aligned}
& S_{2} \leq \frac{12}{5 \log 7} \cdot \frac{\langle m\rangle^{2 \alpha}}{2 \sqrt{d_{m}}} \cdot \log \left(\frac{m+\sqrt{d_{m}}}{m-\sqrt{d_{m}}}\right) \\
& S_{3} \leq \frac{12}{7 \log 7} \cdot \frac{\langle m\rangle^{2 \alpha}}{2 \sqrt{d_{m}}} \cdot \log \left(\frac{m+\sqrt{d_{m}}}{m-\sqrt{d_{m}}}\right) .
\end{aligned}
$$


We give some details only for $S_{2}$. First we write:

$$
S_{2}=\frac{\langle m-1\rangle^{2 \alpha}}{\frac{1}{2}\left(2 m-\frac{3}{2}\right)} \leq \frac{\langle m\rangle^{2 \alpha}}{\frac{1}{2}\left(2 m-\frac{3}{2}\right)} \cdot \frac{\log \left(\frac{m+\sqrt{d_{m}}}{m-\sqrt{d_{m}}}\right)}{2 \sqrt{d_{m}}} \cdot \frac{2 \sqrt{d_{m}}}{\log \left(\frac{m+\sqrt{d_{m}}}{m-\sqrt{d_{m}}}\right)}
$$

Then use

$$
\frac{2 \sqrt{d_{m}}}{\frac{1}{2}\left(2 m-\frac{3}{2}\right)}=\frac{4\left(m-\frac{1}{2}\right)}{2 m-\frac{3}{2}}=2 \cdot \frac{m-\frac{1}{2}}{m-\frac{3}{4}} \leq \frac{12}{5}
$$

and

$$
\frac{1}{\log \left(\frac{m+\sqrt{d_{m}}}{m-\sqrt{d_{m}}}\right)}=\frac{1}{\log \left(\frac{2 m-\frac{1}{2}}{\frac{1}{2}}\right)} \leq \frac{1}{\log 7}
$$

To estimate $S_{4}$ we make an integration by parts:

$$
\begin{aligned}
S_{4} & =\int_{m}^{\infty} \frac{\left(1+x^{2}\right)^{\alpha}}{x^{2}-d_{m}} \\
& =\left[\frac{\left(1+x^{2}\right)^{\alpha}}{2 \sqrt{d_{m}}} \log \left(\frac{x-\sqrt{d_{m}}}{x+\sqrt{d_{m}}}\right)\right]_{m}^{\infty}-\frac{\alpha}{\sqrt{d_{m}}} \int_{m}^{\infty} d x x\left(1+x^{2}\right)^{\alpha-1} \log \left(\frac{x-\sqrt{d_{m}}}{x+\sqrt{d_{m}}}\right) \\
& \leq \frac{\langle m\rangle^{2 \alpha}}{2 \sqrt{d_{m}}} \log \left(\frac{m+\sqrt{d_{m}}}{m-\sqrt{d_{m}}}\right)+\frac{\alpha}{\sqrt{d_{m}}}\left(\sup _{x \geq m} x \log \left(\frac{x+\sqrt{d_{m}}}{x-\sqrt{d_{m}}}\right)\right) \int_{m}^{\infty} x^{2(\alpha-1)} d x .
\end{aligned}
$$

The supremum above is computed by simply noticing that the function $f(x):=$ $x \log \left(\frac{x+a}{x-a}\right)$ is decreasing on $(a, \infty)$. Indeed, its first two derivatives are given by

$$
\begin{aligned}
f^{\prime}(x) & =\log \left(\frac{x+a}{x-a}\right)+\frac{2 x a}{a^{2}-x^{2}} \rightarrow 0 \quad \text { if } x \rightarrow \infty \\
f^{\prime \prime}(x) & =\frac{4 a^{3}}{\left(a^{2}-x^{2}\right)^{2}}
\end{aligned}
$$

Hence

$$
\begin{aligned}
S_{4} & \leq \frac{\langle m\rangle^{2 \alpha}}{2 \sqrt{d_{m}}} \log \left(\frac{m+\sqrt{d_{m}}}{m-\sqrt{d_{m}}}\right)+\frac{\alpha}{1-2 \alpha} \frac{\langle m\rangle^{2 \alpha}}{\sqrt{d_{m}}} \log \left(\frac{m+\sqrt{d_{m}}}{m-\sqrt{d_{m}}}\right) \\
& =\frac{1}{2(1-2 \alpha)} \cdot \frac{\langle m\rangle^{2 \alpha}}{2 \sqrt{d_{m}}} \cdot \log \left(\frac{m+\sqrt{d_{m}}}{m-\sqrt{d_{m}}}\right) .
\end{aligned}
$$

Now taking into account that

$$
\frac{1}{d_{m}} \leq \frac{4}{3 \log 7} \frac{\langle m\rangle^{2 \alpha}}{2 \sqrt{d_{m}}} \log \left(\frac{m+\sqrt{d_{m}}}{m-\sqrt{d_{m}}}\right)
$$


and that $2 \sqrt{d_{m}} \geq \frac{3}{\sqrt{5}}\langle m\rangle$ we collect all the estimates (77.1.4), (7.1.5), (17.1.6), (77.1.7) and plug them into (7.1.1):

$$
\begin{aligned}
\sup _{t \in I_{0}} \sup _{z \in \Gamma_{m}}\left\|K\left(\left(d_{m}+i y\right), t\right)\right\| & \leq\|V\|_{r} \frac{4 \sqrt{5}}{3}\left(\frac{\sqrt{5}}{2}\right)^{\alpha} \cdot \frac{9-6 \alpha}{1-2 \alpha} \frac{\log (4 m-1)}{\langle m\rangle^{1-2 \alpha}} \\
& :=C_{V} \frac{\log (4 m-1)}{\langle m\rangle^{1+\min \{0,2 r\}}} .
\end{aligned}
$$

Note that the constant $C_{V}$ depends on $r$ and has a pathological behaviour at $r=-\frac{1}{2}$. We treat now the case $m=1$. This term contains actually only the terms of the type $S_{3}$ and $S_{4}$, so that:

$$
\sup _{t \in I_{0}} \sup _{z \in \Gamma_{1}}\left\|K\left(\left(d_{1}+i y\right), t\right)\right\| \leq\|V\|_{r}\left(\frac{\sqrt{5}}{2}\right)^{\alpha}\left\{\frac{1}{d_{1}}+2 \sum_{n \geq 1} \frac{\langle 1\rangle^{2 \alpha}}{\left|n^{2}-d_{1}\right|}\right\}
$$

and

$$
\sum_{n \geq 1}^{\infty} \frac{\langle n\rangle^{2 \alpha}}{\left|n^{2}-\frac{1}{4}\right|} \leq \frac{4\langle 1\rangle^{2 \alpha}}{3}+\int_{2}^{\infty} \frac{\langle x\rangle^{2 \alpha}}{x^{2}-\frac{1}{4}}
$$

This leads to

$$
\begin{aligned}
\sup _{t \in I_{0}} \sup _{z \in \Gamma_{1}}\left\|K\left(\left(d_{1}+i y\right), t\right)\right\| & \leq\|V\|_{r}\left(\frac{\sqrt{5}}{2}\right)^{\alpha}\left(4+\frac{8\langle 1\rangle^{2 \alpha}}{3}+\frac{\langle 1\rangle^{2 \alpha}}{1-2 \alpha} \log 3\right) \\
& \leq C_{V} \frac{\log 3}{\langle 1\rangle^{1+\min \{0,2 r\}}} .
\end{aligned}
$$

Inspecting (7.1.9) and (7.1.10) it is clear that one has for any $m \geq 1$

$$
\sup _{t \in I_{0}} \sup _{z \in \Gamma_{m}}\left\|K\left(\left(d_{m}+i y\right), t\right)\right\| \leq C_{V} \frac{\log (4 m-1)}{\langle m\rangle^{1+\min \{0,2 r\}}} .
$$

From this estimate one can identify $m^{*}$ such that for any $m>m^{*}$ we have $\sup _{t \in I_{0}} \sup _{z \in \Gamma_{m}} \| K\left(\left(d_{m}+\right.\right.$ $i y), t) \| \leq 1$.

Now we turn to the estimation of $\sup _{t \in I_{0}} \int_{\Gamma_{m}}\left\|R_{0}(z, t)\right\| \cdot\|K(z, t)\| d y$. The resolvent is easily estimated as

$$
\left\|R_{0}(z, t)\right\| \leq \frac{1}{\sqrt{A^{2}+y^{2}}}
$$

where we introduced the notation $A:=\left(m-\frac{1}{2}\right)^{2}-\left(m-\frac{3}{4}\right)^{2}$. Since $\sup _{t \in I_{0}}\|K(z, t)\| \leq$ 
$4\|K(z, 0)\|_{H S}$ we have

$$
\begin{aligned}
& \sup _{t \in I_{0}} \int_{\Gamma_{m}}\left\|R_{0}(z, t)\right\| \cdot\|K(z, t)\| d y \\
\leq & \left.4\|V\|_{r}\left(\frac{\sqrt{5}}{2}\right)^{\alpha} \int_{-\infty}^{\infty} d y \frac{1}{\sqrt{A^{2}+y^{2}}}\left(\sum_{n_{1} \in \mathbb{Z}} \frac{\left\langle n_{1}\right\rangle^{2 \alpha}}{\sqrt{B_{n_{1}}^{2}+y^{2}}} \cdot \sum_{n_{2} \in \mathbb{Z}} \frac{\left\langle n_{2}\right\rangle^{2 \alpha}}{\sqrt{B_{n_{2}}^{2}+y^{2}}}\right)^{\frac{1}{2}}\right)^{\frac{1}{2}} \\
\leq & 4\|V\|_{r}\left(\frac{\sqrt{5}}{2}\right)^{\alpha}\left(\int_{-\infty}^{\infty} d y \sum_{n_{1} \in \mathbb{Z}} \frac{\left\langle n_{1}\right\rangle^{2 \alpha}}{\sqrt{A^{2}+y^{2}} \sqrt{B_{n_{1}}^{2}+y^{2}}}\right)^{\frac{1}{2}} \text { by Cauchy }- \text { Schwartz } \\
\cdot & \left(\int_{-\infty}^{\infty} d y \sum_{n_{2} \in \mathbb{Z}} \frac{\left.d n_{2}\right\rangle^{2 \alpha}}{\sqrt{A^{2}+y^{2}} \sqrt{B_{n_{2}}^{2}+y^{2}}}\right)^{\alpha} \\
= & 4\|V\|_{r}\left(\frac{\sqrt{5}}{2}\right)^{\alpha} \sum_{n \in \mathbb{Z}}\langle n\rangle^{2 \alpha} \int_{-\infty}^{\infty} \frac{d y}{\sqrt{A^{2}+y^{2}} \sqrt{B_{n}^{2}+y^{2}}} \\
:= & 4\|V\|_{r}\left(\frac{\sqrt{5}}{2}\right)^{\alpha} \sum_{n \in \mathbb{Z}}\langle n\rangle^{2 \alpha} I\left(A, B_{n}\right) .
\end{aligned}
$$

In the above calculation we used as well the notation:

$$
B_{n}:=n^{2}-d_{m}
$$

The integral $I\left(A, B_{n}\right)$ is estimated as follows (in our case $B_{n}>A$ always)

$$
\begin{aligned}
I\left(A, B_{n}\right) & \leq \frac{1}{B_{n}^{1-\delta}} \int_{-\infty}^{\infty} d y \frac{1}{\sqrt{A^{2}+y^{2}} \cdot\left(A^{2}+y^{2}\right)^{\frac{\delta}{2}}} \\
& =\frac{1}{A^{\delta} B_{n}^{1-\delta}} \int_{-\infty}^{\infty} d p \frac{1}{\left(1+p^{2}\right)^{\frac{1+\delta}{2}}} \\
& =\frac{1}{A^{\delta} B_{n}^{1-\delta}} \cdot \frac{\sqrt{\pi} \Gamma(\delta / 2)}{\Gamma((1+\delta) / 2)}=\frac{C_{\delta}}{A^{\delta} B_{n}^{1-\delta}},
\end{aligned}
$$

where we made the substitution $p=y / A$. On the other hand $B_{n}$ obeys the following estimate

$$
B_{n}=\left|n^{2}-d_{m}\right| \leq \max \left\{n^{2}, d_{m}\right\} \leq \max \left\{\langle n\rangle^{2},\left\langle d_{m}\right\rangle^{2}\right\} \leq\langle n\rangle^{2}\left\langle d_{m}\right\rangle^{2}
$$

from where

$$
I\left(A, B_{n}\right) \leq C_{\delta} \frac{\langle n\rangle^{2 \delta}\left\langle d_{m}\right\rangle^{2 \delta}}{A^{\delta} B_{n}} .
$$

Using the fact that the function $g(m):=\frac{\left\langle d_{m}\right\rangle^{2}}{A\langle n\rangle}$ is decreasing so that $\max _{m \in \mathbb{Z}} g(m)=$ $g(0)=4$ one has $\frac{\left\langle d_{m}\right\rangle^{2 \delta}}{A^{\delta}} \leq 4\langle m\rangle^{2 \delta}$. Thus

$$
\begin{aligned}
& \sup _{t \in I_{0}} \int_{\Gamma_{m}}\left\|R_{0}(z, t)\right\| \cdot\|K(z, t)\| d y \leq 16\|V\|_{r}\left(\frac{\sqrt{5}}{2}\right)^{\alpha} C_{\delta}\langle m\rangle^{2 \delta} \sum_{n \in \mathbb{Z}} \frac{\langle n\rangle^{2(\alpha+\delta)}}{\left|n^{2}-d_{m}\right|} \\
& \leq C_{V} C_{\delta} \frac{\langle m\rangle^{4 \delta} \log (4 m-1)}{\langle m\rangle^{1+\min \{0,2 r\}}} \quad 0<\delta<\frac{1}{4}-\frac{\alpha}{2} .
\end{aligned}
$$


The last condition on $\delta$ assures that the RHS vanishes in the limit $m \rightarrow \infty$. We further optimise the result by using the inequality

$$
C(\delta) \leq \frac{3}{\delta}
$$

and taking

$$
\delta=\frac{1}{4 \log \langle m\rangle}<\frac{1}{4}-\frac{\alpha}{2}
$$

from where it follows that $\langle m\rangle \geq e^{\frac{1}{(1-2 \alpha)}}$ and

$$
\delta^{-1}\langle m\rangle^{4 \delta}=4 e \log \langle m\rangle
$$

Then

$$
\sup _{t \in I_{0}} \int_{\Gamma_{m}}\left\|R_{0}(z, t)\right\| \cdot\|K(z, t)\| d y \leq C_{V} \frac{\log ^{2}(4 m-1)}{\langle m\rangle^{\min \{1,1+2 r\}}}
$$

The estimates (7.1.11) and (7.1.20) lead to

$$
\sup _{t \in I_{0}}\left(\sup _{z \in \Gamma_{m}}\|K(z, t)\|+\int_{\Gamma_{m}}\left\|R_{0}(z, t)\right\| \cdot\|K(z, t)\| d y\right) \leq C_{V} \frac{\log ^{2}\langle 4 m-1\rangle}{\langle m\rangle^{\min \{1,1+2 r\}}} .
$$

In what concerns the estimates on $\tilde{\Gamma}_{m}$ one has to follow the same steps as above, the result being that $m$ is pushed to $m+\frac{1}{2}$ Since $\left\langle m+\frac{1}{2}\right\rangle \leq \sqrt{2}\langle m\rangle$ and $\log (4 m+1) \leq$ $2 \log (4 m-1)$ one can work only with $b(m)$. To prove (2.43) and (2.44) one has to use the first resolvent identity:

$$
\begin{aligned}
\|K(z, t)\| & \leq\left\|K\left(d_{m}, t\right)\right\| \cdot\left\|\frac{R_{0}(z, t)}{R_{0}\left(d_{m}, t\right)}\right\| \\
& \leq\left\|K\left(d_{m}, t\right)\right\|\left(1+\frac{\left|z-d_{m}\right|}{\inf _{z \in \gamma_{m}} \operatorname{dist}\left(z, \sigma\left(H_{0}(t)\right)\right)}\right) .
\end{aligned}
$$

Since

$$
\begin{array}{r}
\sup _{z \in \gamma_{n}}\left|z-d_{m}\right|=\sqrt{5}(m-1) \\
\inf _{t \in I_{0}} \inf _{z \in \gamma_{m}} \operatorname{dist}\left(z, \sigma\left(H_{0}(t)\right)\right) \leq \frac{1}{4}\left(2 m-\frac{11}{4}\right)
\end{array}
$$


from where (2.44) follows. Finally let us prove (7.1.2)

$$
\begin{aligned}
\inf _{t \in I_{0}} \inf _{n \in \mathbb{Z}}\left|\frac{(n+t)^{2}-d_{m}}{n^{2}-d_{m}}\right| & =\inf _{t \in I_{0}} \inf _{n \in \mathbb{Z}}\left|\frac{(n+t)-\sqrt{d_{m}}}{n-\sqrt{d_{m}}}\right|\left|\frac{(n+t)+\sqrt{d_{m}}}{n+\sqrt{d_{m}}}\right| \\
& \geq \inf _{t \in I_{0}} \inf _{n \in \mathbb{Z}}\left|\frac{(n+t)-\sqrt{d_{m}}}{n-\sqrt{d_{m}}}\right| \inf _{t \in I_{0}} \inf _{n \in \mathbb{Z}}\left|\frac{(n+t)+\sqrt{d_{m}}}{n+\sqrt{d_{m}}}\right| \\
& =\inf _{t \in I_{0}} \inf _{n \in \mathbb{Z}}\left|1+\frac{t}{n-\sqrt{d_{m}}}\right| \inf _{t \in I_{0}} \inf _{n \in \mathbb{Z}}\left|1+\frac{t}{n+\sqrt{d_{m}}}\right| \\
& \geq\left(1-\sup _{t \in I_{0}} \sup _{n \in \mathbb{Z}}\left|\frac{t}{n-\sqrt{d_{m}}}\right|\right)\left(1-\sup _{t \in I_{0}} \sup _{n \in \mathbb{Z}}\left|\frac{t}{n+\sqrt{d_{m}}}\right|\right) \\
& \geq\left(1-\sup _{n \in \mathbb{Z}}\left|\frac{\frac{1}{4}}{n-\sqrt{d_{m}}}\right|\right)\left(1-\sup _{n \in \mathbb{Z}}\left|\frac{\frac{1}{4}}{n+\sqrt{d_{m}}}\right|\right) \\
& =\left(1-\frac{1}{4 \operatorname{dist}\left(\sqrt{d_{m}}, \mathbb{Z}\right)}\right)^{2}=\frac{1}{4} .
\end{aligned}
$$

The proof of Lemma 2.3 is finished.

\subsection{The Sz-Nag̈̈ tranformation}

For convenience we give here the well known [15] Sz-Nagÿ lemma on unitary equivalence of orthogonal projections.

Lemma 7.2.1: Let $P, Q$ be orthogonal projections in a Hilbert space $\mathcal{H}$ satisfying

$$
\|P-Q\|<1
$$

If $U$ is defined by

$$
U=\left(1-(P-Q)^{2}\right)^{-\frac{1}{2}}(P Q+(1-P)(1-Q))
$$

then

$$
U^{*}=U^{-1}
$$

and

$$
P=U Q U^{*}
$$

Notice also that

$$
\left(1-(P-Q)^{2}\right)^{-\frac{1}{2}}=1+\sum_{j \geq 1} \frac{(2 j-1) ! !}{j ! 2^{j}}(P-Q)^{2 j} .
$$




\subsection{The remainder of order $p$ for the Dyson series}

In this appendix we review a method to estimate the remainder of order $p$ for the Dyson series of $\widehat{\Omega}_{P, m}$ encountered in Section 5 . Let $\mathcal{E}$ be the space of $C^{\infty}$ operatorvalued functions on $I_{0}$ taking values in $\mathcal{B}\left(\mathbb{C}^{2}\right)$. Thus $\forall X \in \mathcal{E}, \forall l \in \mathbb{N}$

$$
\|X\|_{\infty}:=\sup _{t \in I_{0}}\left\|\partial_{t}^{l} X(t)\right\|<\infty
$$

since $I_{0}$ is compact and $t \rightarrow \partial_{t}^{l} X(t)$ is continuous. We define the operator $K: \mathcal{E} \rightarrow \mathcal{E}$ by the following relation

$$
(K X)(t):=-i \int_{t_{0}}^{t} \widehat{V}_{P}(s) X(s) d s, \quad \widehat{V}_{P}(t):=\left(\begin{array}{cc}
0 & b(t) \\
b(t) & 0
\end{array}\right),
$$

where $b(t):=\hat{V}(2(m-1)) e^{2 i(m-1)\left(t^{2}-t_{0}^{2}\right)}$. The Dyson series with remainder of order $p$ reads as

$$
\widehat{\Omega}_{P, m}^{0}\left(t_{1}, t_{0}\right)=\sum_{k=0}^{m-1} K^{k}(t) \mathrm{id}+K^{m}(t) \widehat{\Omega}_{P, n_{0}}^{0}\left(t_{1}, t_{0}\right) .
$$

Here $K^{k}$ denotes $K \circ K \circ \ldots \circ K \mathrm{k}$ times. We start by proving a technical lemma.

Lemma 7.3.1 Let $a:=m-1$. Then for all $X \in \mathcal{E}$ it holds

$$
\|K X\|_{\infty} \leq \frac{|b|}{\sqrt{a}} 2 \sqrt{6}\|X\|_{\infty}+|b| \frac{\log \left(\frac{\sqrt{a}}{2 \sqrt{6}} \vee 1\right)}{a}\|\dot{X}\|_{\infty}
$$

where we used the notation $m \vee n:=\max \{m, n\}$

Proof: We shall use the canonical basis in $\mathcal{B}\left(\mathbb{C}^{2}\right)$ which reads as follows

$$
\Pi_{1,1}:=\left(\begin{array}{cc}
1 & 0 \\
0 & 0
\end{array}\right) \quad \Pi_{2,2}:=\left(\begin{array}{cc}
0 & 0 \\
0 & 1
\end{array}\right) \quad \Pi_{1,2}:=\left(\begin{array}{cc}
0 & 1 \\
0 & 0
\end{array}\right) \quad \Pi_{2,1}:=\left(\begin{array}{ll}
0 & 0 \\
1 & 0
\end{array}\right)
$$

Also, let $\chi_{-}, \chi_{0}$ et $\chi_{+}$be the characteristic functions corresponding to the intervals $\left[-\frac{1}{4},-\varepsilon\right],[-\varepsilon, \varepsilon]$ and $\left[\varepsilon, \frac{1}{4}\right]$ where $0<\varepsilon<\frac{1}{4}$. Then we have

$$
K X=K \chi_{-} X+K \chi_{0} X+K \chi_{+} X .
$$

One gets immediately

$$
\left\|K \chi_{0} X\right\|_{\infty} \leq 2 \varepsilon|b|\|X\|_{\infty}
$$

For $K \chi_{ \pm} X$ we use the identity $K \chi_{ \pm} X=K \chi_{ \pm} \Pi_{1,1} X+K \chi_{ \pm} \Pi_{2,2} X$. We shall treat in detail only the term $K \chi_{-} \Pi_{2,2} X$, the estimates for the remaining parts being completely similar. Then with the notation $m \wedge n:=\min \{m, n\}$ we have

$$
\begin{aligned}
\left(K \chi_{-} \Pi_{2,2} X\right)(t) & =-i \int_{t_{0}}^{t \wedge-\varepsilon} b(s) \Pi_{1,2} X(s) d s \\
& =-\int_{t_{0}}^{t \wedge-\varepsilon} \operatorname{4ias} b(s) \frac{\Pi_{1,2} X(s)}{4 a s} d s \\
& =-\left[\frac{b(s) \Pi_{1,2} X(s)}{4 a s}\right]_{t_{0}}^{t \wedge-\varepsilon}+\int_{t_{0}}^{t \wedge-\varepsilon} b(s) \partial_{s}\left(\frac{\Pi_{1,2} X(s)}{4 a s}\right) d s,
\end{aligned}
$$


since $\dot{b}(s)=4 i a s b(s)$. The first term is estimated as follows

$$
\left\|\left[b(s) \frac{\Pi_{1,2} X(s)}{4 a s}\right]_{t_{0}}^{t \wedge-\varepsilon}\right\| \leq \frac{|b|}{2 a \varepsilon}\left\|\Pi_{1,2} X\right\|_{\infty} .
$$

For the second term

$$
\int_{t_{0}}^{t \wedge-\varepsilon} b(s) \partial_{s}\left(\frac{\Pi_{1,2} X(s)}{4 a s}\right) d s=\int_{t_{0}}^{t \wedge-\varepsilon} b(s)\left(-\frac{\Pi_{1,2} X(s)}{4 a s^{2}}+\frac{\Pi_{1,2} \dot{X}(s)}{4 a s}\right) d s,
$$

and consequently

$$
\begin{aligned}
\left\|\int_{t_{0}}^{t \wedge-\varepsilon} b(s) \partial_{s}\left(\frac{\Pi_{1,2} X(s)}{4 a s}\right) d s\right\| & \leq\left\|\Pi_{1,2} X\right\|_{\infty} \int_{t_{0}}^{-\varepsilon} \frac{|b|}{4 a s^{2}} d s+\left\|\Pi_{1,2} \dot{X}\right\|_{\infty} \int_{t_{0}}^{-\varepsilon} \frac{|b|}{4 a|s|} d s \\
& \leq \frac{|b|}{4 a \varepsilon}\left\|\Pi_{1,2} X\right\|_{\infty}-\frac{|b| \log (4 \varepsilon)}{4 a}\left\|\Pi_{1,2} \dot{X}\right\|_{\infty} .
\end{aligned}
$$

Finally we find

$$
\|K X\|_{\infty} \leq|b|\left(\left(2 \varepsilon+\frac{1}{a \varepsilon}\right)\|X\|_{\infty}-\frac{\log (4 \varepsilon)}{a}\|\dot{X}\|_{\infty}\right)
$$

and chosing $\varepsilon=\sqrt{3}(2 a)^{-\frac{1}{2}} \wedge \frac{1}{4}$ we arrive at the announced result.

Using Lemma 7.3.1 and the identity $\frac{d}{d t}\left(K^{p-1} X\right)(t)=\widehat{V}(t)\left(K^{p-2} X\right)(t)$ one can get the general result:

$$
\text { if } \begin{aligned}
p \geq 2, \quad\left\|K^{p} X\right\|_{\infty} & \leq \frac{|b|}{\sqrt{a}} c\left\|K^{p-1} X\right\|_{\infty}+|b| \frac{\log \frac{\sqrt{a}}{c}}{a}\left\|\widehat{V} K^{p-2} X\right\|_{\infty} \\
& \leq A\left\|K^{p-1} X\right\|_{\infty}+B\left\|K^{p-2} X\right\|_{\infty},
\end{aligned}
$$

with the notations

$$
c=2 \sqrt{6}, A:=\frac{|b|}{\sqrt{a}} c, B=|b|^{2} \frac{\log \frac{\sqrt{a}}{c} \vee 1}{a} .
$$

By looking at the expansion of $\Omega_{P, m}^{0}\left(t_{1}, t_{0}\right)$ it is clear that we have to solve (7.3.11) in the case $X=\Omega_{P, m}^{0}$. However, in order to estimate as well $\widehat{\Omega}_{P, m}^{0,(p)}$ the case $X=1$ would be also usual. We consider then the following two sequences for $\forall p \geq 2$

$$
x_{p}^{(k)}=A x_{p-1}^{(k)}+B x_{p-2}^{(k)} \quad \text { with } x_{0}^{(k)}=1 \quad \text { and } x_{1}^{(k)} \text { given. }
$$

Here $k=1,2$ labels the considered case, namely $x_{p}^{(1)}=\left\|K^{p}\right\|$ and $x_{p}^{(2)}=\left\|K_{p} \widehat{\Omega}_{P, m}^{0}\right\|$. We know that $x_{p}$ is a linear combination of two geometric series $p \rightarrow r_{ \pm}^{p}$. More explicitly $x_{p}=\alpha_{+} r_{+}^{p}+\alpha_{-} r_{-}^{p}$ where $r_{ \pm}$are the solutions of the characteristic equation $r^{2}-A r-B=0$. Denoting with $\Delta:=\sqrt{A^{2}+4 B}$ one finds

$$
r_{ \pm}=\frac{A \pm \sqrt{\Delta}}{2} \text {. }
$$


The coefficients of the linear combination $\alpha_{ \pm}$are solutions of the equation

$$
\left(\begin{array}{cc}
1 & 1 \\
r_{+} & r_{-}
\end{array}\right)\left(\begin{array}{c}
\alpha_{+} \\
\alpha_{-}
\end{array}\right)=\left(\begin{array}{c}
x_{0} \\
x_{1}
\end{array}\right) \Longleftrightarrow \alpha_{ \pm}= \pm \frac{x_{1}-r_{\mp} x_{0}}{\sqrt{\Delta}} .
$$

Now for the case 1 we have by direct calculation $\alpha_{ \pm}^{(1)}=\frac{r_{ \pm}}{\sqrt{\Delta}}$ and then

$$
x_{p}^{(1)}=\frac{r_{+}^{p+1}-r_{-}^{p+1}}{\sqrt{\Delta}}=\sum_{k=0}^{p} r_{+}^{k} r_{-}^{p-k}=r_{+}^{p} \sum_{k=0}^{p}\left(\frac{r_{-}}{r_{+}}\right)^{p-k} .
$$

For the second case we use the superposition principle. Let $y$ the sequence defined by the relation as $x$ with the initial conditions $y_{0}=0$ et $y_{1}=B$. Then we have $x^{(2)}=x^{(1)}+y$. Since $y_{p}=\beta_{+} r_{+}^{p}+\beta_{-} r_{-}^{p}$ with $\beta_{ \pm}= \pm B \Delta^{-\frac{1}{2}}$ one has

$$
x_{p}^{(2)}=x_{p}^{(1)}+\frac{B}{\sqrt{\Delta}}\left(r_{+}^{p}-r_{-}^{p}\right)=x_{p}^{(1)}+B \sum_{k=0}^{p-1} r_{+}^{k} r_{-}^{p-k} .
$$

We proceed to the estimates by noticing that $\left|r_{-}\right|<r_{+}$which allows to write

$$
\left|\sum_{k=0}^{p} r_{+}^{k} r_{-}^{p-k}\right|=\left|r_{+}^{p} \sum_{k=0}^{p}\left(\frac{r_{-}}{r_{+}}\right)^{p-k}\right| \leq r_{+}^{p} \frac{1}{1-\frac{\left|r_{-}\right|}{r_{+}}}=\frac{r_{+}^{p+1}}{r_{+}-\left|r_{-}\right|}=\frac{r_{+}^{p+1}}{A}
$$

and consequently

$$
\left|x_{p}^{(1)}\right| \leq \frac{r_{+}^{p+1}}{A} \quad\left|x_{p}^{(2)}\right| \leq \frac{r_{+}^{p+1}}{A}+\frac{B}{A} r_{+}^{p} .
$$

Using these results we obtain

$$
\| K^{p} \text { id } \| \leq \frac{r_{+}}{A} r_{+}^{p} \leq\left(1+\frac{\sqrt{B}}{A}\right) B^{\frac{p}{2}}\left(1+\frac{A}{\sqrt{B}}\right)^{p}
$$

where

$$
\frac{\sqrt{B}}{A}=\frac{1}{4 \sqrt{3}}\left(\log \left(\frac{a}{24} \vee 1\right)\right)^{\frac{1}{2}} .
$$

The estimate on $x_{p}^{(2)}$ gives

$$
\left\|K^{p} \widehat{\Omega}_{P, m}^{0}\right\| \leq \frac{r_{+}^{p+1}}{A}+\frac{B}{A} r_{+}^{p} \leq\left(1+\frac{\sqrt{B}}{A}+\frac{B}{A}\right) B^{\frac{p}{2}}\left(1+\frac{A}{\sqrt{B}}\right)
$$

where

$$
\frac{B}{A}=\frac{1}{4 \sqrt{6}} \frac{|b|}{\sqrt{a}} \log \left(\frac{a}{24} \vee 1\right)
$$

Therefore we have proved:

Theorem 7.3.2 Let $\widehat{\mathcal{U}}_{P, m}$ and $\widehat{\mathcal{U}}_{P, m}^{0}$ as defined in Section 5. $a=m-1$ and $b=\hat{V}(2(m-1))$. Then there exists $m_{0}$ and a constant $C_{m_{0}}$ such that the Dyson 
series for $\widehat{\Omega}_{P, m}^{0}\left(t, t_{0}\right)=\widehat{\mathcal{U}}_{P, m}^{0}\left(t, t_{0}\right)^{*} \widehat{\mathcal{U}}_{P, m}^{0}$ converges uniformly with respect to $t$ for every $t \in I_{0}$ and that for any $m>m_{0}$ one has the following estimate

$$
\left\|\widehat{\Omega}_{P, m}^{0,(p)}\right\|_{\infty} \leq C_{m_{0}}|b|^{p} \sqrt{\log a}\left(\frac{\log a}{a}\right)^{\frac{p}{2}} .
$$

The remainder of order $p$ satisfy the estimate

$$
\left\|K^{p} \widehat{\Omega}_{P, m}^{0}\right\|_{\infty} \leq 2 C_{m_{0}}|b|^{p} \sqrt{\log a}\left(\frac{\log a}{a}\right)^{\frac{p}{2}} .
$$

Acknowledgments G. Nenciu and V. Moldoveanu would like to thank for the warm hospitality and financial support encountered along their visits at CPT Marseille and Université de Toulon et du Var. Parts of the draft were written during the visits of F. Bentosela and P. Duclos at Institute of Mathematics of the Romanian Academy. The financial support from the Provence Alpes Côtes d'Azur region, Romanian Academy - CNRS (France) exchange agreement, Grant CERES 38/02, Grant CNCSIS and Eurrommat Programme is hereby acknowledged.

\section{References}

[1] Abramowitz, M.I, Stegun, I. A., "Handbook of Mathematical Functions" (Dover, New York, 1972).

[2] Ao, P., "Absence of localization in energy space of a Bloch electron driven by a constant electric force", Phys.Rev. B, 41, 3998-4001, (1990).

[3] Asch, F, Bentosela, F., Duclos, P., Nenciu, G., "On the Dynamic of Cristal Electrons, high Momentum Regime", J. Math. Anal. Appl. 256, 99-114 (2001).

[4] Asch, J., Duclos, P., Exner, P., "Stability of driven systems with growing gaps, quantum rings, and Wannier ladders", J. Stat. Phys. 92, 1053-1070 (1998).

[5] Avron, J. E. and Nemirovski, J., "Quasienergies, Stark Hamiltonians and Energy Growth for Driven Quantum Rings", Phys. Rev. Lett., 68, 2212-2215 (1992).

[6] Avron, J. E., Seiler R., Yaffe, L. G., "Adiabatic theorems and applications to the quantum Hall effect", Comm. Mat. Phys. 110, 33-49 (1987).

[7] Bentosela, F., Carmona, R., Duclos, P., Simon, B., Souillard, B. and R. Weder, "Schrödinger operators with an electric field and random or deterministic potentials", Comm. Math. Phys., 88, 387-397 (1983).

[8] Bentosela, F., Grecchi, V. and Zironi, F., " Stark-Wannier states in disordered systems", Phys. Rev. B 31, 6909-6912 (1985). 
[9] Bentosela, F., Grecchi, V. and Zironi, F., "Oscillations of Wannier Resonances" , Phys. Rev. Lett. 50, 84-86 (1983).

[10] Bentosela, F., Grecchi, V. and Zironi, F., "Approximate ladder of resonances in a semi-infinite crystal", J. Phys. C 15, 7119-7131 (1982).

[11] Bhatia, R., Rosenthal, P., Bull. London Math. Soc. 29, 1-21 (1997).

[12] Blatter, G., Browne, D. A., "Zener tunneling and localizations in small conducting rings", Phys. Rev. B, 37, 3856-3880, (1988).

[13] Deift, P., Killip, R., "On the Absolutely Continuous Spectrum of OneDimensional Schrödinger Operators with Square Summable Potentials", Comm. Math. Phys. 203, 341-347 (2001).

[14] Enss, V. and Veselić, K, "Bound States and Propagating States for Timedependent Hamiltonians, Ann. Inst. H. Poincaré", 39, 159-191, (1983).

[15] Kato, T., "Perturbation theory of linear operators", (Springer-Verlag, New York, 1966).

[16] Killip, R., "Perturbations of one-dimensional Schrödinger operators preserving the absolutely continuous spectrum", mp-arc preprint, (2001).

[17] A. Kiselev, A., "Absolutely continuous spectrum of perturbed Stark operators", Trans. Am. Math. Soc. 352, 243-256 (2000).

[18] Krein, S. G., "Linear Differential Equations in Banach Spaces", Nauka Moscow 1967, (American Mathematical Society, Providence, 1971).

[19] Lenstra, D. and van Haeringen, W., "On the problem of DC conductivity at $\mathrm{T}=0 \mathrm{~K}$ in a one-dimensional system of finite length: calculation of the scattering rate in case of weak disorder", J. Phys. C 14, 5293-5303 (1981).

[20] Lenstra, D. and van Haeringen, W., "Elastic Scattering in a Normal-Metal Loop Causing Resistive Electronic Behavior", Phys. Rev. Lett., 56, 1623-1626 (1986).

[21] Martin, Ph. A., Nenciu, G., "Semi-classical inelastic S-matrix for one dimensional N-states systems" Rev. Math. Phys. 7, 193-242, (1995).

[22] Nenciu, A. and Nenciu, G., "Dynamics of Bloch electrons in external electric fields I. Bounds for interband transitions and effective Wannier Hamiltonians", J. Phys. A 14, 2817-2827 (1981).

[23] Nenciu, A. and Nenciu, G., "Dynamics of Bloch electrons in external fields II. The existence of Stark-Wannier ladder resonances", J. Phys A 15, 3313-3328 (1982). 
[24] Nenciu, G, "Dynamics of band electrons in electric and magnetic fields: rigorous justification of the effective Hamiltonians", Rev. Mod. Phys. 63, 91-127 (1991).

[25] Nenciu, G., "Linear adiabatic theory. Exponential estimates", Comm. Math. Phys. 152, 479-496 (1993).

[26] Perelman, G., "On the Absolutely Continuous Spectrum of Stark Operators", Comm. Math. Phys., 234 359-381 (2003).

[27] Reed, M., and Simon, B., "Methods of Modern Mathematical Physics II: Fourier Analysis. Self-Adjointness" (Academic, New York, 1975).

[28] Reed, M., and Simon, B., "Methods of Modern Mathematical Physics IV: Analysis of Operators" (Academic, New York, 1978).

[29] Simon, B., "Quantum mechanics for hamiltonians defined as quadratic forms", (Princeton University press, New Jersey 1971).

[30] Voisin, P., J. Bleuse, C. Bouche, S. Gaillard, C. Alibert, and A. Regreny, "Observation of the Wannier-Stark Quantization in a Semiconductor Superlattice", Phys. Rev. Lett. 61, 1639-1642, (1988).

[31] Zener, C., . Proc.R.Soc.London Ser. A 145, 523- (1934).

[32] Yosida, K., "Functional Analysis", Springer (1968). 